\title{
Laboratory Directed Research and Development Program FY 2005
}

April 2006 


\section{DISCLAIMER}

This document was prepared as an account of work sponsored by the United States Government. While this document is believed to contain correct information, neither the United States Government nor any agency thereof, nor The Regents of the University of California, nor any of their employees, makes any warranty, express or implied, or assumes any legal responsibility for the accuracy, completeness, or usefulness of any information, apparatus, product, or process disclosed, or represents that its use would not infringe privately owned rights. Reference herein to any specific commercial product, process, or service by its trade name, trademark, manufacturer, or otherwise, does not necessarily constitute or imply its endorsement, recommendation, or favoring by the United States Government or any agency thereof, or The Regents of the University of California. The views and opinions of authors expressed herein do not necessarily state or reflect those of the United States Government or any agency thereof or The Regents of the University of California.

Lawrence Berkeley Laboratory is an equal opportunity employer. 


\section{Report on \\ Ernest Orlando Lawrence Berkeley National Laboratory}

\section{Laboratory Directed Research and Development Program}

FY 2005

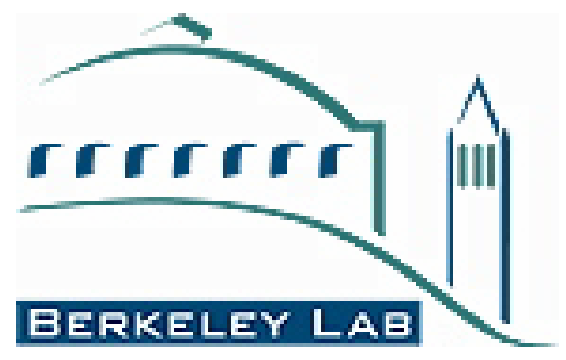

Ernest Orlando Lawrence Berkeley National Laboratory Berkeley, CA 94720
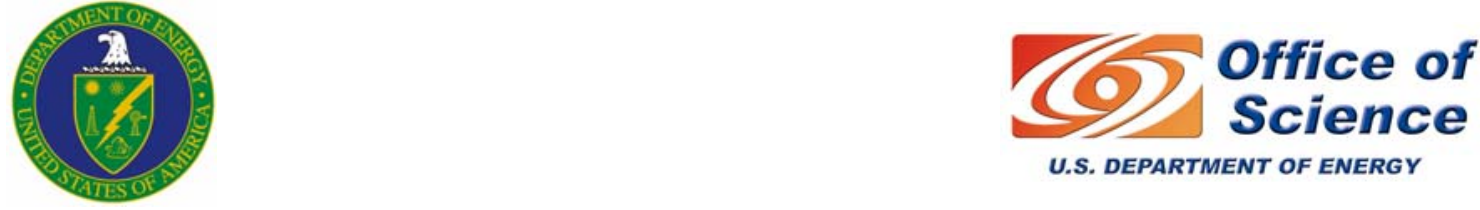

U.S. DEPARTMENT OF ENERGY 



\section{Table of Contents}

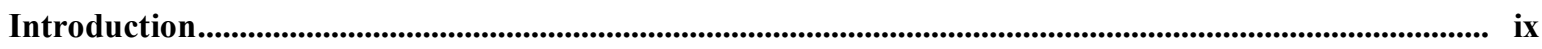

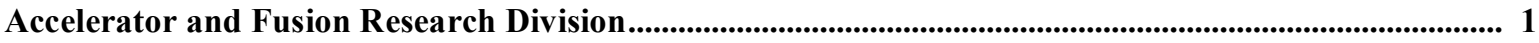

Steven Lidia

Alexander Zholents

John Corlett

Stephen Leone

Howard Padmore

Robert Schoenlein

Russell Wells

Grant Logan

Jonathan Wurtele

Wim Leemans

Esmond Ng

Robert Ryne

Miguel Furman

Jean-Luc Vay

Max Zolotorev

Eugene Commins

Peter Denes

Zahid Hussain

Gennadi Lebedev

Steven Lidia

Jim O'Neil

David Robin

Fernando Sannibale

Robert Schoenlein

Robert Vogel

Weishi Wan
Critical Accelerator Technologies for Advanced Light Sources .... 1

Enabling High Energy Density Physics at LBNL............................

2

Optimal Solvers for Infinite-Dimensional Hamiltonian Systems ... 3

Electron Production and Collective Field Generation in Intense

Particle Beams ........................................................................ 4

Low Energy Spread Electron Source.............................................. 5

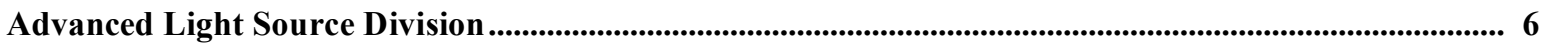

Malcolm Howells

Coherent X-ray Diffraction Imaging (CXDI)

Janos Kirz

John Spence

Uwe Weierstall

Henry Chapman

Chris Jacobsen

Howard Padmore

A. Minor

Janos Kirz

Lensless Imaging of Yeast Cells ...

Michael Martin

Left-handed Nanoscale Meta-Materials: Towards the Optical

Zhao Hao

Domain

Eric Anderson

J. Alexander Liddle

Eli Rotenberg

Aaron Bostwick

NanoARPES: A New Detector for Nanometer-scale Electronic

Structure Measurements 
Andreas Scholl

Ultra-fast Magnetization Dynamics

Anthony Young

Jun Feng

Joachim Stöhr

Howard Padmore

Russell Wilcox

Robert Schoenlein

High Average Power Laser Amplifier.

Chemical Sciences Division

Musahid Ahmed

Christopher Chang

Stephen Leone

Daniel Neumark

Thomas Rescigno

C. William McCurdy

T. Don Tilley
Gas Phase Studies of the Building Blocks of Life

Oxygen Catalysis through Proton and Electron Control:

Biomimetic Chemistry for Renewable Energy

Time-resolved X-ray Absorption Spectroscopy of Metalloporphyrins.

Spectroscopy and Dynamics of Pure and Doped Helium

Nanodroplets

Advanced Computational Methods for Photon-Molecule

Collision Processes

New Approach for the Catalysis Conversion of Methane and Other Inert Hydrocarbons.

\section{Computing Sciences}

(National Energy Research Scientific Computing Center, Computational Research. and Information Technologies and Services Divisions )

Olivier Chevassut

Cryptographic Foundations for New Generation Distributed

Abdelilah Essiari

Systems

Bernd Hamann

Interactive Visualization Methods for Exploration and

Comparison of Multi-Billion Base Pair Sequence Data

Wolfgang Hoschek

Peer-to-Peer Resource Discovery Framework

Optimizing Genomic Data Storage for Wide Accessibility .....

Jonathan Carter

Damian Hazen

Juan Meza

Parallel Methods for Robust Optimization and Uncertainty

Quantification

Enhancing Commodity Scalar Processors with Vector

Components for Increased Scientific Productivity

John Shalf

KatherineYelick

William Kramer

Ali Pinar

Advanced Computational Tools for Electric Power Systems

Bernard Lesieutre

Chao Yang

Juan Meza

Vanessa Lopez

Vaibhav Donde

Raquel Romano

Paul Hargrove

KatherineYelick
Statistical Feature Modeling for Scientific Data Via Basis

Decomposition 25

Evaluation of Computer Architecture Alternatives 26 
Lisa Alvarez-Cohen

Terry Hazen

Sally Benson

James Bishop

Paul Luke

Sharon Borglin

Christopher Campbell

William Stringfellow

Bailey Green

Eleanor Wozei

Allen Grayson

Donald DePaolo

Benjamin Gilbert

Norman Miller

Nigel Quinn

Larry Dale

Helen $\mathrm{He}$

George Moridis

Stefan Finsterle

Eric Sonnenthal

Garrison Sposito

Kurt Nihei

Steven Pride

Ernest Majer

Carl Steefel
Real-time PCR With Reverse Transcription for Quantification of Chlorinated Solvent Degradation ................................................. 27

Scientific Basis for Advanced Geologic Storage Technologies ..... 28

Autonomous Sensors for Ocean Dissolved Organic Matter ........... 29

Development of Biosensors for Endocrine Disrupting

Compounds in Agricultural Watersheds

Micro-characterization and Chemical Micro-dynamics of Atmospheric Mineral Dust.............................................................. 31

Behavior and Impact of Nanoparticles in the Environment............ 32

The California Water and Energy Systems: an Approach for Addressing Future Crises

Next Generation Codes for Modeling Subsurface Processes

Advancing the Next Generation of Rock-Fluid Imaging and

Stimulation Technologies.

Biogeochemical Reaction Rates and Pathways in Porous Media... 36

Engineering Division

Peter Denes

Novel Imaging Detectors for Materials and Biology Research .......

37

Howard Padmore

Environmental Energy Technologies Division

Marc Fisher

Nitash Balsara

Jean Frechet

Steve Johnson

John Kerr

Gao Liu

Rachel Segalman

Nitash Balsara

Kyungoul Baek

Robert Kostecki

Samuel Mao

Richard Russo
Constraining Ammonia Emission Inventories for Control of Air

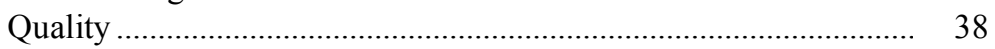

Highly Efficeint PLED Through Polymer Morphology Control.... 39
Novel High-Temperature Membranes and Electrocatalysts

Structures for Fuel Cells

Nanostructured Cathodes for Efficient Organic LEDs

41 
Surabi Menon

John Newman

Jayant Sathaye

Mark Levine

Lynn Price

Stephane de la Rue

du Can

Michael McNeil

Shyam Menon

Virginie Letschert

Phil Price

Jonathan Sinton

Nan Zhou
Evaluating Aerosol Effects on Regional and Global Energy and Water Budgets

Analysis of High-temperature Polymer-electrolyte Fuel Cell

Phenomena

Long-term Global Energy Demand and Carbon Emissions

Scenarios

Genomics Division

Eugene Myers

Making the Most of Sequencing: Improved Assembly and

Comparative Annotation

Eddy Rubin

Transgenic Mouse Production and Identification of New Genes

in Transgenic Mice

Life Sciences Division

Manfred Auer

Felicia Betancourt

Mina Bissell

Mark Biggin

Jian Jin

Ursula Schulze-Gahmen

Susan Celniker

John Conboy

Robert Glaeser

Jian Jin

Roar Kilaas

Kenneth Downing

Eva Nogales

Manfred Auer

Joe Gray

Bo Hang

Anton Guliaev

William Jagust

G. Steven Martin Mina Bissell
Molecular Electron Microscope Tomograph

Response of Chromatin Structures in Mammary Epithelial Cells to Microenvironmental Cues 48

High Throughput Strategy to Identify Protein Complexes

Modeling Human Disease in Drosophila melanogaster 50

Unmasking the Human Genome Alternative Splicing Program..... 51

Improved Phase Contrast for Cryo-EM of Biological Machines and Subcelluar Structure

Functional Interpretation of Cancer Genomes

Computational Analysis of Structural Impacts of Exocyclic

DNA Adducts on Specificity and Efficiency of Human Repair

Enzymes

Neuroimaging with Advanced Molecular Probes. 55

Imaging Signaling Networks in Normal and Malignant Tissue ..... 56 


\author{
Andrea Cavallerii \\ Robert Schoenlein \\ Nigel Browning \\ Ulrich Dahmen
}

Oscar Dubón

J. Alexander Liddle

Daryl Chrzan

Ehud Isacoff

Dirk Trauner

Richard Kramer

\section{J. Alexander Liddle}

Nitash Balsara

Joseph Orenstein

Ramamoorthy Ramesh

Dung-Hai Lee

Joel Moore

Yuri Suzuki

Ivo Souza

Steven Louie

Yuri Suzuki

J. Alexander Liddle

Andreas Scholl

Hendrik Ohldag

Ashvin Vishwanath
Ultrafast Measurements of Charge-ordering/ disordering in

Manganites: Development of a Femtosecond Electron

Diffraction Probe

Techniques of Sample Controls for a Transmission Electron Aberration-Corrected Microscope

Ordering and Shape Control of Epitaxial Germanium

Nanostructures on Metal-patterened Silicon

Light-activated and Ion Channels and Transporters

Nanoscale Lithography to Guide Self-assembly for the Creation of Functional, Hierarchical Nanostructures

Science and Technology of Quantum Materials

62

Computational and Theoretical Studies of Bulk and Nano Solid

Systems

Complex Oxide Nanostructures: Understanding Surface,

Interface, and Finite Size Effects Through Nanofabrications

Experimental Signatures of Fractionalized Phases and Phase Transitions in Strongly Correlated Electronic Systems.

Nuclear Science Division

Kevin Lesko

Research and Development for Double Beta Decay

Experiments.

Margaret McMahan

Establishing New Capabilities in Nuclear Astrophysics and

Radiation Biology Using Neutrons at the 88" Cyclotron

Larry Phair

Improved Spectroscopy of Weakly Bound States in Nuclei

68

Physical Biosciences Division

Arup Chakraborty

Adam Arkin

Phillip Colella

Philip Geissler

Teresa Head-Gordon

Gavin Crooks

Thomas Earnest

Daniel Fletcher

Jan Liphardt
Organization of Cellular Components on Signaling and Function: Theory and Computation as Essential Complements to Genetic and Biochemical Experiments

Statistical Dynamics of Protein Evolution

High-throughput Production of Proteins and Protein Complexes .. 71

Synthetic Cytoskeleton: Protrusive Structures for Reconstituted Cell Motility

Rapid Characterization of Microbial RNAs Using Artificial Nanopores 
Robert Tjian Development of Techniques for the Study of Large

Macromolecular Complexes Using X-ray Crystallography.....

Physics Division..

Marco Battaglia

Advanced Monolithic Silicon Pixel Sensors.

75

Stuart Freedman

Designing a Novel Reactor Neutrino Oscillation Experiment for

Karsten Heeger

Measuring the Unknown Mixing Angle $\Theta_{13}$

Richard Kadel

Kam-Biu Luk

Hitoshi Murayama

New Directions for Theoretical Physics at the TeV-Scale

77

Cross-Divisional

\section{A. Paul Alivisatos Carolyn Bertozzi \\ Fanqing Chen \\ Joe Gray \\ Thomas McKone \\ Phillip Geissler}

William Lester Graham Fleming Michael Frenklach

JayMarx
Studies of the Health Effects of Nanostructured Materials

78

Microscopic Theory of Protein Surface: Structure, Response, and Design

Development and Application of Quantum Monte Carlo (QMC)

Methods to Biological Systems.

Development of Key Physics and Technical Approaches for the Production of Intense Rare Isotope Beams 


\section{Introduction}

The Ernest Orlando Lawrence Berkeley National Laboratory (Berkeley Lab or LBNL) is a multi-program national research facility operated by the University of California for the Department of Energy (DOE). As an integral element of DOE's National Laboratory System, Berkeley Lab supports DOE's missions in fundamental science, energy resources, and environmental quality. Berkeley Lab programs advance four distinct goals for DOE and the nation:

- To perform leading multidisciplinary research in the computing sciences, physical sciences, energy sciences, biosciences, and general sciences in a manner that ensures employee and public safety and protection of the environment.

- To develop and operate unique national experimental facilities for qualified investigators.

- To educate and train future generations of scientists and engineers to promote national science and education goals.

- To transfer knowledge and technological innovations and to foster productive relationships among Berkeley Lab's research programs, universities, and industry in order to promote national economic competitiveness.

Berkeley Lab's research and the Laboratory Directed Research and Development (LDRD) program support DOE's Strategic Goals that are codified in DOE's September 2003 Strategic Plan, with a primary focus on Advancing Scientific Understanding. For that goal, the Fiscal Year (FY) 2005 LDRD projects support every one of the eight strategies described in the plan. In addition, LDRD efforts support the goals of Investing in America's Energy Future (six of the fourteen strategies), Resolving the Environmental Legacy (four of the eight strategies), and Meeting National Security Challenges (unclassified fundamental research that supports stockpile safety and nonproliferation programs). The LDRD supports Office of Science strategic plans, including the 20 year Scientific Facilities Plan and the Office of Science Strategic Plan. The research also supports the strategic directions periodically under review by the Office of Science Program Offices, such as strategic LDRD projects germane to new research facility concepts and new fundamental science directions.

Berkeley Lab LDRD program also play an important role in leveraging DOE capabilities for national needs. The fundamental scientific research and development conducted in the program advances the skills and technologies of importance to our Work For Others (WFO) sponsors. Among many directions, these include a broad range of health-related science and technology of interest to the National Institutes of Health, breast cancer and accelerator research supported by the Department of Defense, detector technologies that should be useful to the Department of Homeland Security, and particle detection that will be valuable to the Environmental Protection Agency.

The Berkeley Lab Laboratory Directed Research and Development Program FY2005 report is compiled from annual reports submitted by principal investigators following the close of the fiscal year. This report describes the supported projects and summarizes their accomplishments. It constitutes a part of the LDRD program planning and documentation process that includes an annual planning cycle, project selection, implementation, and review.

The Berkeley Lab LDRD program is a critical tool for directing the Laboratory's forefront scientific research capabilities toward vital, excellent, and emerging scientific challenges. The program provides the resources for Berkeley Lab scientists to make rapid and significant contributions to critical national science and technology problems. The LDRD program also advances Berkeley Lab's core competencies, foundations, and scientific capability, and permits exploration of exciting new opportunities. All projects are work in forefront areas of science and technology. Areas eligible for support include the following:

- Advanced study of hypotheses, concepts, or innovative approaches to scientific or technical problems;

- Experiments and analyses directed toward "proof of principle" or early determination of the utility of new scientific ideas, technical concepts, or devices; and

- Conception and preliminary technical analyses of experimental facilities or devices.

The LDRD program supports Berkeley Lab's mission in many ways. First, because LDRD funds can be allocated within a relatively short time frame, Berkeley Lab researchers can support the mission of the Department of Energy (DOE) and serve the needs of the nation by quickly responding to forefront scientific problems. Second, LDRD enables Berkeley Lab to attract and retain highly qualified scientists and to support their efforts to carry out worldleading research. In addition, the LDRD program also supports new projects that involve graduate students and postdoctoral fellows, thus contributing to the education mission of Berkeley Lab.

Berkeley Lab has a formal process for allocating funds for the LDRD program. The process relies on individual 
scientific investigators and the scientific leadership of Berkeley Lab to identify opportunities that will contribute to scientific and institutional goals. The process is also designed to maintain compliance with DOE Orders, in particular DOE Order 413.2A, dated January 8, 2001. From year to year, the distribution of funds among the scientific program areas changes. This flexibility optimizes Berkeley Lab's ability to respond to opportunities.

Berkeley Lab LDRD policy and program decisions are the responsibility of the Laboratory Director. The Director has assigned general programmatic oversight responsibility to the Deputy Director. Administration and reporting on the LDRD program is supported by the Directorate's Office for Planning and Development. LDRD accounting procedures and financial management are consistent with the Laboratory's accounting principles and stipulations under the contract between the University of California and the Department of Energy, with accounting maintained through the Laboratory's Chief Financial Officer.

In FY2005, Berkeley Lab was authorized by DOE to establish a funding ceiling for the LDRD program of $\$ 18.0$ M, which equates to about $3.1 \%$ of Berkeley Lab's FY2005 projected operating and capital equipment budgets. This funding level was provided to develop new scientific ideas and opportunities and allow the Berkeley Lab Director an opportunity to initiate new directions. Budget constraints limited available resources, however, so a little less than $\$ 12.9 \mathrm{M}$ was expended for operating and $\$ 0.7 \mathrm{M}$ for capital equipment ( $2.7 \%$ of actual Berkeley Lab FY2005 costs).

In FY2005, scientists submitted 171 proposals, requesting over $\$ 33.6 \mathrm{M}$ in total funding. Eighty-one projects were funded, with awards ranging from $\$ 50 \mathrm{~K}$ to $\$ 860 \mathrm{~K}$. These projects are identified in the Table of Contents. 


\title{
Accelerator and Fusion Research Division
}

LB04019

\author{
Critical Accelerator Technologies for Advanced Light Sources \\ Principal Investigator(s): S. Lidia, A. Zholents, J. Corlett, S. Leone, H. Padmore, \\ R. Schoenlein, R. Wells
}

Project Description

This project combines analytical, computational, and experimental approaches to develop concepts and technologies related to the production of x-rays in future accelerator-based facilities. We address the critical areas of x-ray production in self-amplified spontaneous emission and cascaded-harmonic free electron lasers, ultra-stable synchronization and timing techniques, high-power and high-brightness electron beam sources, and emittance control in high-brightness electron beams. These schemes provide enhanced performance and capabilities over existing methods for EUV and soft x-ray production.

\section{Accomplishments}

We conceived and developed novel ESASE techniques to generate extremely high peak current ( 1-20 kA) micro-bunches within an electron beam bunch, with only limited beam quality degradation due to collective effects. Utilizing two distinct wavelength lasers $(1200 \mathrm{~nm}$ and $1600 \mathrm{~nm}$ ) we have demonstrated peak current enhancement and gain length reduction in the LCLS undulator, thereby easing the stringent requirements on the LCLS electron beam quality. GINGER simulations have demonstrated saturation in the LCLS case with a $50 \mathrm{~m}$ undulator, producing sub-picosecond photon pulses with reduced shot-to-shot fluctuations and synchronization with the seed laser. Separately, we have developed a method for electron beam conditioning based on established optical technology that overcomes the severe limitations in previously proposed systems. Included as an add-on device to x-ray free electron lasers, this conditioner also eases the electron beam quality requirements while significantly shortening the undulator length needed for saturation of the x-ray pulse.

Addressing a very significant issue in short pulse x-ray light sources, we designed and built a fiber optic synchronization link as a proof of concept for timing distribution. Adapting techniques from the radiotelescope community, we have demonstrated improved phase sensitivity by orders of magnitude and achieved sub-femtosecond jitter and drift resolution. Over a $100 \mathrm{~m}$ fiber length, we have demonstrated a significant reduction of drift to less than 1 femtosecond per hour with peak-to-peak jitter less than 2.6 femtoseconds.

We have developed advanced techniques for simulating high brightness electron beams. Fully 3D, massively parallel start-to-end simulations that account for nonlinear space charge forces, beam emission, image forces near photocathodes, beams with large aspect ratios, longitudinal and transverse wakefields in accelerator structures, and models of coherent space charge (CSR) effects in magnetic chicanes allow us to predict and model the generation of high brightness electron beams required for $\mathrm{x}$-ray free electron lasers and advanced light sources.

We have performed Monte Carlo simulations of photoemission in $\mathrm{Cs}_{2} \mathrm{Te}$ films that allow us to model surface and transport effects that may limit beam quality in high brightness electron beam production. We have measured quantum efficiency enhancement effects in $\mathrm{Cu}$ films, due to the vectorial photoelectric effect that may signal a nonlocal contribution to the conductivity tensor.

We have completed engineering studies that have demonstrated successful thermal management during high average power operation of an rf photoinjector cavity, enabling electron beam production up to $10 \mathrm{kHz}$ repetition rates. 


\section{LDRD-HEDP-FY2005 report: Enabling High Energy Density Physics at LBNL Principle Investigator(s): Grant Logan, Jonathan Wurtele, Wim Leemans}

\section{Project Description}

High Energy Density Physics (HEDP) is an emerging field of science to understand the behavior of matter in extreme conditions of temperature and density. HEDP experiments offer us the potential to resolve fundamental questions about plasmas in several regimes important to our understanding of the universe. Our goal is to advance the technological basis of HEDP by developing computational tools and necessary diagnostic capabilities to conduct exciting high energy density plasma experiments using laboratory lasers and accelerators. Our proposal is to a) study new directions in HEDP experiments driven by intense focused particle beams using theory and advanced computation necessary for HEDP, and b) develop cutting edge short pulse x-ray diagnostics for HEDP scientific exploration. This will be accomplished by two tasks directed at computer codes and laser/x-ray scattering experiments:

Task 1: Conduct broad surveys of existing experimental techniques used to study equation of state and other properties of high energy density plasmas. Use state-of-the-art 2-D and 3-D hydrodynamic codes and equation of state models to design experimental HEDP targets that can best advance understanding of HED plasma properties using available drivers.

Task 2: Using the l'OASIS laser facility and with particle, fluid and hybrid codes, study production of x-ray beams from laser-accelerated electron beams for HEDP target diagnostics. Measure and characterize X-rays emitted via betatron oscillations of laser accelerated electron beams in the ion plasma channels using various conventional detector systems including a streak camera and a unique polarization-sensitive pixilated detector. A radiation emission process is being included in a particle-in-cell code to predict and simulate the x-ray source performance.

\section{Accomplishments}

Task 1 We collaborated with LLNL physicists (J. Bernard and M. Marinak) on obtaining, running and modifying as needed a world-class HEDP simulation code, HYDRA. We evaluated various computational codes which could be used or modified to study ion beam driven HEDP. HYDRA is clearly the best choice for us: we have access to a fully three dimensional code that can handle ions and laser pulses. We have used HYDRA in initial studies of ion heated targets at .1 and .01 solid density. It confirms theoretical estimates that underdense foams can be used in conjunction with $\sim 1 \mathrm{~ns}$ ion beams to create fairly uniformly heated targets. Specific models appropriate to planned ion accelerators are ongoing. We are currently working (Martin and Collela) on an LBNL code that will not have all the physics modules of a major code such as HYDRA, but will have advanced AMR numerics and be available to be run at LBNL without access control restrictions. Task 2 We have started detailed modeling of the properties of X-ray radiation from laseraccelerated electron beams. Part of this study was published in IEEE Trans. on Plasma Science by W.P. Leemans et al. in 2005. The first method relies on emission of x-ray photons via betatron oscillations that the electrons undergo while being accelerated in plasma channels. We are in the process of studying this radiation on our $100 \mathrm{MeV}$-class accelerator at L'OASIS. The second method relies on Thomson scattering an intense laser off the laser accelerated electron beams. We are in contact with the NIF Directorate to discuss this approach. To further enhance our predictive capabilities, we are implementing radiation emission processes in particle-in-cell codes. These codes have been effectively used by our group to model the laser based acceleration (see Nature paper by C.G.R. Geddes et al., 431, 2004, which was also the cover story of that Nature issue), and the added feature will also allow us to model the x-ray emission processes. 
Optimal Solvers for Infinite-Dimensional Hamiltonian Systems

Principal Investigators: Esmond Ng and Robert D. Ryne

\section{Project Description}

The focus of this project is to develop optimal discrete models for infinitedimensional Hamiltonian systems. We use symplectic split-operator methods in the time domain, finite element-based and wavelet-based spatial decompositions, and examine both Hamiltonian and Lagrangian discretizations.

This work was motivated in part by the great success of symplectic integrators for classical finite-dimensional Hamiltonian systems. These have become indispensable for studying long-term behavior including the dynamics of charged particles in plasmas, the dynamics of stars in gravitational systems, and the dynamics of atoms and molecules in materials.

Accomplishments

In FY05, our efforts were focused primarily on the simultaneous compression and denoising and spatial discretization aspects of the project. We succeeded in developing and applying both wavelet- and finite element-based discretization schemes, as well as in testing extensively the resulting algorithms and software.

The principal accomplishment for the project in FY05 was the successful integration of the newly implemented three-dimensional wavelet-based Poisson solver into the ImpactT suite of beam dynamics codes. This allowed us to carry out extensive testing in simulations of actual physical systems of a number of spatial discretization schemes and compression/denoising concepts that can be used in modeling infinitedimensional Hamiltonian systems in higher dimensions. We also addressed the optimization of algorithms in a way that allows for simultaneous de-noising of the data sets and reduction in the computing time and storage requirements. In addition, we gained significant expertise in the design and application of highly efficient, effectively diagonal preconditioners for elliptic operators in wavelet bases. The results obtained and insights developed in this setting are immediately applicable to the design of solvers for the hyperbolic PDE's representative of the infinite-dimensional Hamiltonian systems under study. In addition, the algorithms developed so far have immediate application to modeling synchrotron radiation and its effects on the dynamics of beams in accelerators and light sources.

On the finite element-based discretization side of the project, we concentrated our efforts on designing two-dimensional families of higher-order finite elements that have across-the-boundary continuity properties optimized for modeling systems whose solutions are known to be continuously differentiable. A secondary focus was on identifying finite element families whose members have degrees of freedom that can be identified with the dynamical variables of a physical system under study, thus facilitating the process of imposing initial and/or boundary conditions for the discretized model. 
Electron Production and Collective Field Generation in Intense Particle Beams Principal Investigators: Miguel Furman, Jean-Luc Vay (this is a joint LBNL-LLNL LDRD)

Project Description

Electron cloud effects (ECEs) are increasingly recognized as important, but incompletely understood, dynamical phenomena that can severely limit the performance of present electron colliders; the next generation of high-intensity rings, such as PEP-II upgrade, LHC, the SNS, and the SIS 100/200; or future high-intensity heavy ion accelerators such as envisioned in Heavy Ion Inertial Fusion (HIF). Deleterious effects include ion-electron instabilities, emittance growth, particle loss, increase in vacuum pressure, excessive heat load at the vacuum chamber walls, and interference with certain beam diagnostics.

This joint LDRD project has laid essential groundwork for launching a comprehensive R\&D program including experiments, theory and simulations to better understand the phenomena, establish the essential parameters, and develop mitigating mechanisms. We have developed insights into the essential processes and models of the relevant physics, and implemented these models in computational production tools that can be used for self-consistent study of the effect on ion beams. We have validated the models and tools through comparison with experimental data, including data from new diagnostics developed as part of this work and validated on the High-Current Experiment (HCX) at LBNL and the STS500 test stand at LLNL. We have started to apply these models and diagnostic techniques to High-Energy Physics (HEP) and other advanced accelerators.

Accomplishments

New functionalities have been added to our Particle-In-Cell code WARP: a module to generate neutrals desorbed by beam ion impacts at the wall, and a module to track impact ionization of the gas by beam ions or electrons. The new electron mover developed over the three years of the LDRD has been further tested on a "textbook" case and a break-through speedup of 1-2 orders of magnitude in computation time has been demonstrated. The formation of a virtual cathode due to massive generation of electrons from beam ions hitting a wall was investigated using XOOPIC (UCB) and the necessary temporal and spatial resolution assessed.

With the benefit of the new additions and the electron mover speedup, WARP simulations met our objective of replicating results of the HCX experiments when electrons from an end wall are permitted to flood the beam. Semi-quantitative agreement was obtained on the observation of a "Z" shaped distortion of the horizontal beam phase space, with agreement on the sign and shape of the distortion, and to a lesser degree on its magnitude. Close-quantitative agreement was obtained on the observation of oscillations in the current collected in a ring placed between two quadrupole magnets, with very good agreement in the amplitude and period of the oscillation. The code was also applied to the modeling of the Large Hadron Collider (LHC) where the first ever 3-D modeling of one bunch interacting with electron clouds in a full FODO cell was performed. Further LHC simulations have been requested by CERN; this post-LDRD work is now funded by the US-LARP program.

We developed measurements of each source of electrons and their accumulation during a pulse. We also developed experimental methods to control, and limit the accumulation of, electrons from various sources that will enable more quantitative and controlled future experiments on electrons and their effects. These fundamental physics advances provide a basis for evaluating and extending the current limits of present and future major accelerators. 
Low Energy Spread Electron Source

Principal Investigators: Max Zolotorev, Eugene D. Commins, Peter Denes, Zahid Hussain, Gennadi Lebedev, Steven Lidia, Jim Oneill, David Robin, Fernando Sannibale, Robert

Schoenlein, Robert Vogel, Weishi Wan.

\section{Project Description}

The purpose of the project is to explore a novel approach for a pulsed electron source that has the potential to approach the quantum-limited brightness and lowering the effective source temperature from the current $\sim 100 \mathrm{meV}$ to $\sim 10 \mu \mathrm{eV}$. Such a source can open a wide range of novel applications that utilize angstrom-scale spatial resolution and $\mu \mathrm{eV}$-scale energy resolution. Examples include angstrom-scale resolution electron microscopy, electron holography and investigations of dynamics on the ps time scale using pump-probe techniques. The technology available within the proposed budget will constrain the effective temperature to the meV-range. In this multi-year LDRD we will demonstrate this proof of principle by constructing the source and measuring its temperature to the achievable limit.

In the scheme, an effusive source generates a beam of neutral Cesium atoms. These atoms are then excited to a Rydberg level very close to ionization $\left(\sim 10^{-5} \mathrm{eV}\right)$ in the interaction region (IR) defined by the overlapping of 3 laser beams. Two of the lasers are CW while the third is pulsed with a rep-rate of $10 \mathrm{MHz}$. The parameters are tuned to produce an average of 1 Rydberg excited atom per pulse. After the laser pulse, a delay $(\sim 40 \mathrm{~ns})$ is introduced to allow the electron in the excited atom to travel far from the ion $(\sim 70 \mu \mathrm{m})$ reaching the turning point of its orbit so as to lose practically all of its kinetic energy. At this point, a pulsed voltage is applied to finally ionize the atom and to deliver the electron to the optical system for the final acceleration and for the energy analysis. Last, a second pulsed electric field removes the remaining ion from the IR. In this way the Coulomb interaction effects are minimized and a very high brightness electron beam is obtained.

Accomplishments

During this first year of LDRD, the source characteristics have been fully defined and a parameter list for the whole system has been completed. A systematic and detailed investigation including quantitative evaluation of all the physical phenomena involved in the operation of the source has been done. Few examples: comparison between quantum and classical mechanics calculations; evaluation of the effects induced by, thermal velocity of the atom beam, finite duration of the laser pulse, scattered light induced photoemission, scattering between the atoms in the Cs beam, stray electric and magnetic fields, ....

The three lasers for the Cs atom excitation scheme have been purchased. The two CW systems are already in operation and frequency stabilized by our house-developed feedbacks. The work on the pulsed laser is in progress.

The Cs source design is in its final phase and the construction phase will start soon.

Calculations and simulations of the electron optics parameters (electron acceleration and focusing, ion clearing) are in progress for defining the requirements on field quality.

We have also built a test chamber for measuring the electron photoemission from Cs layers deposited on different metals for the defining the proper material for the construction of the source components. First measurements have been already performed. 


\title{
Advanced Light Source Division
}

LB04013

\section{COHERENT X-RAY DIFFRACTION IMAGING (CXDI)}

\author{
Investigators: M. R. Howells ${ }^{1}$, J. Kirz ${ }^{1}$, J. C. H. Spence ${ }^{2}$, U. Weierstall ${ }^{2}$, H. R. Chapman ${ }^{3}$, \\ Chris Jacobsen ${ }^{4}$, H. A. Padmore ${ }^{1}$, A. Minor ${ }^{1}$. \\ ${ }^{1}$ Lawrence Berkeley National Laboratory \\ ${ }^{2}$ Arizona State University \\ ${ }^{3}$ Lawrence Livermore National Laboratory \\ ${ }^{4}$ State University of New York at Stony Brook
}
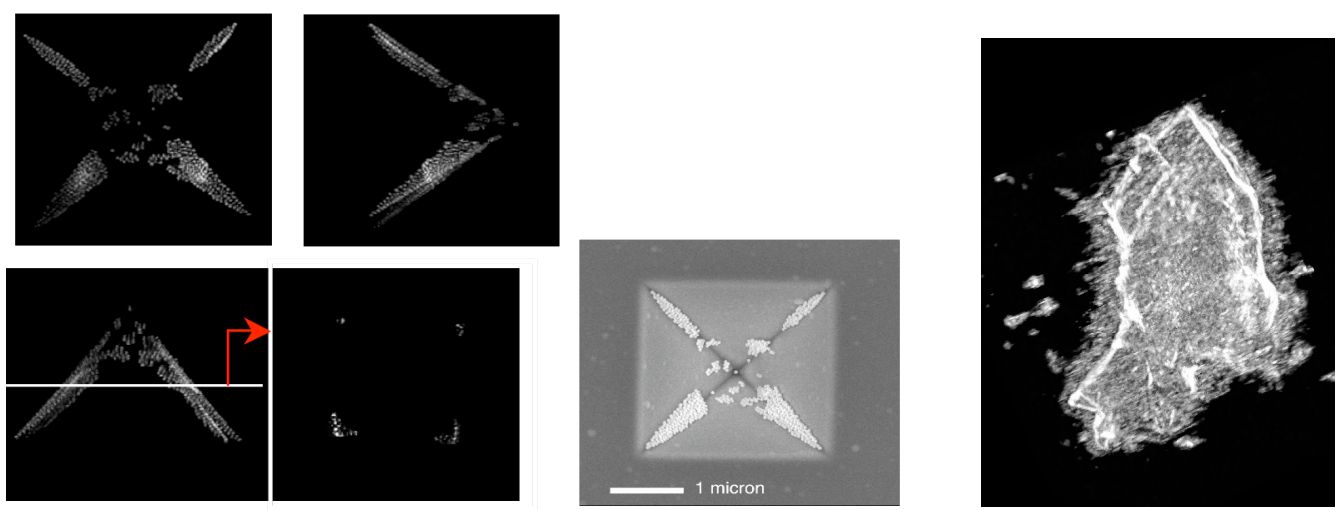

Fig 1. At the left are shown three views of, and one section through, a pyramid-shaped threedimensional test object of maximum dimension $2.2 \mu \mathrm{m}$. The object consists of an assembly of $50 \mathrm{~nm}$ gold balls held together by a shaped silicon nitride membrane. At the center is a scanning-electron-microscope picture of of the same object looking down into the hollow pyramid. At the right is one view of a three-dimensional image of an aerogel particle (maximum dimension $2 \mu \mathrm{m}$ ). The particle consists of Tantalum oxide foam of about $1 \%$ of bulk density. The resolution in both experiments is about $10 \mathrm{~nm}$.

In the last few years all four of the United States Department of Energy synchrotron radiation facilities have been pursuing an idea for a new type of three-dimensional microscope that was first demonstrated in two dimensions at Brookhaven in 1999. The idea is Coherent X-ray Diffraction Imaging (CXDI), which works by recording a tilt series of diffraction patterns of the object and then recovering the three-dimensional image by computation using a phase-retrieval algorithm. The motivation for developing a new imaging method must always be that it will enable important and otherwise impractical new investigations to be carried out. In the case of CXDI the new challenge that is already beginning to be met is to image objects in the one to about twenty micron size range in three dimensions at a resolving power of around $1000 \times 1000 \times 1000$ voxels. Such objects are too big to be imaged at good resolution in the transmission electron microscope while their features are too small to be imaged in the light microscope. Success in this new type of imaging will have a decisive impact on the growing activities of nano-science and nanotechnology.

With two years of LDRD funding we have already succeeded in reconstructing threedimensional images (Fig 1) from diffraction patterns recorded using coherent X-rays of 0.7 $\mathrm{keV}$ energy produced by Advanced Light Source beam line 9.0.1. The "pyramid" data were recorded at 140 view angles (one-degree intervals) and the aerogel data at 280 view angles (half-degree intervals). The exposure time was about eight hours in both cases. 


\section{Lensless Imaging of Yeast Cells \\ Principal Investigator: Janos Kirz}

\section{Project Description}

Our motivation for developing diffraction based imaging of non-crystalline biological specimens is based on its unique characteristics, that unlike other forms of imaging, the resolution is not limited by the technology of the fabrication of optical elements such as zone plates. Our goal is to develop the technique, and image frozen hydrated yeast cells (Saccharomyces cerevisiae) in three dimensions at about $15 \mathrm{~nm}$ resolution. The improvement in resolution compared to what has been accomplished (or is likely to be accomplished in the foreseeable future) by other techniques (such as the XM1 microscope) is expected to provide insight into the life cycle and the interrelation of the organelles within this important model organism. We collaborate on the preparation of the specimens and on the interpretation of the results with some of the noted experts in yeast biology, such as Prof. Aaron Neiman of Stony Brook.

Data collection makes use of the ALS 9.0.1 undulator beamline built by Malcolm Howells. We brought and installed a custom built experimental apparatus, built by the Stony Brook group. This apparatus is permanently mounted on the beamline, and its use is shared by the Livermore/ASU/ALS group. It is designed to accommodate specimens between room temperature and liquid nitrogen temperature. It is capable of collecting diffraction data sets over a wide range of tilt angles under computer control. Graduate student David Shapiro collected data on freeze-dried yeast cells, and developed additional data collection and analysis software. More recently graduate student Enju Lima took over further development and data collection from frozen hydrated yeast cells.

\section{Accomplishments}

During the past year we succeeded in collecting complete 2D data sets from freeze-dried yeast cells. In collaboration with Veit Elser and Pierre Thibault of Cornell University, we were able to reconstruct the data to better than $30 \mathrm{~nm}$ resolution. These results formed the $\mathrm{Ph}$. $\mathrm{D}$. thesis of David Shapiro, and a paper that appeared recently in PNAS.

Enju Lima developed techniques for the preparation of frozen hydrated specimens, and collected extensive 2D and 3D data sets which are currently being analyzed. 


\section{Left-Handed Nanoscale Meta-Materials: Towards the Optical Domain}

Michael C. Martin, Zhao Hao, Erik Anderson, Alex Liddle

\section{Project Description}

Artificial meta-materials can be made that have a negative index of refraction, which allows a homogeneous flat slab of the material to behave as a "perfect" lens, possibly even creating sub-diffraction limited focusing. The meta-materials consist of split-ring resonators which provide a negative $\mu$, and metal strips which provide a negative $\varepsilon$. The goal of this LDRD is to fabricate and measure left-handed nano-scale meta-materials taking advantage of nano-fabrication expertise within the Center for X-Ray Optics.

\section{Accomplishments}

We have begun the micro- and nano-fabrication of these meta-material structures using gold on thin $\mathrm{SiN}$ windows (see examples of split ring resonators in the Figure below, with dimensions as indicated). The SiN is thin enough that these are nearly free-
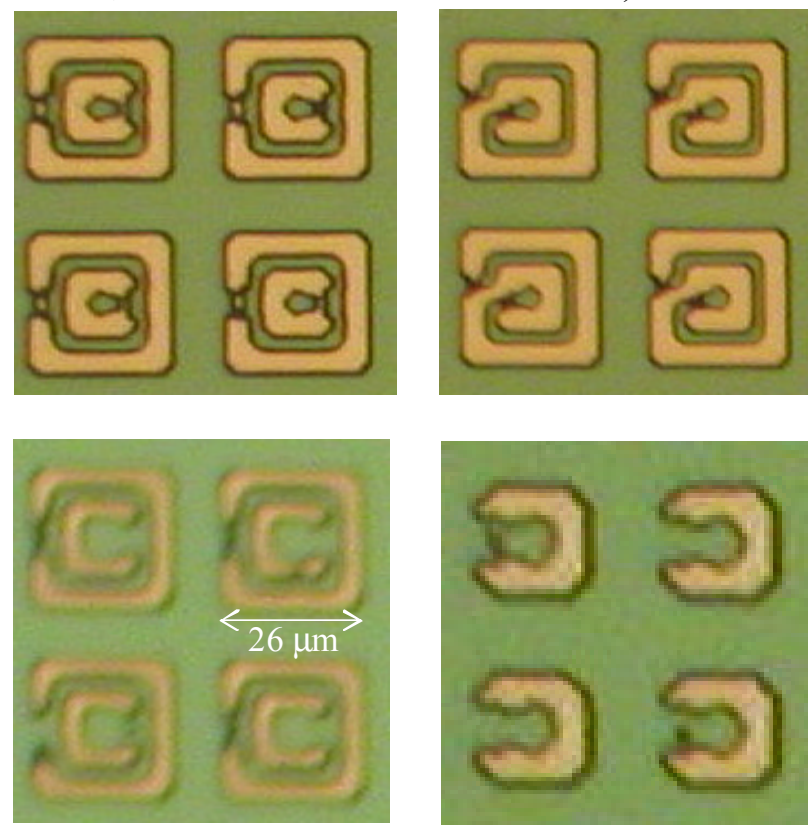

$\mathrm{THz}$ ) is well predicted by the EM simulations.

We have continued to push to smaller dimensions with structures having $60 \mathrm{~nm}$ smallest dimensions using a nanowriter, and developing international collaborations to fully exploit these exciting meta-materials. standing structures allowing us to fully characterize the index of refraction properties as a function of wavelength using ALS BL 1.4.2, 1.4.3, and 1.4.4.

We measured the polarized farinfrared $(\mathrm{THz})$ spectral response of these meta-materials. Samples are measured in reflectance with variable angles of incidence between 5 and $80^{\circ}$. The figure below shows an example of the measured and simulated magnetic resonance from the double split ring resonator in the inset. The observed resonance frequency at $\sim 37 \mathrm{~cm}^{-1}(\sim 1.1$

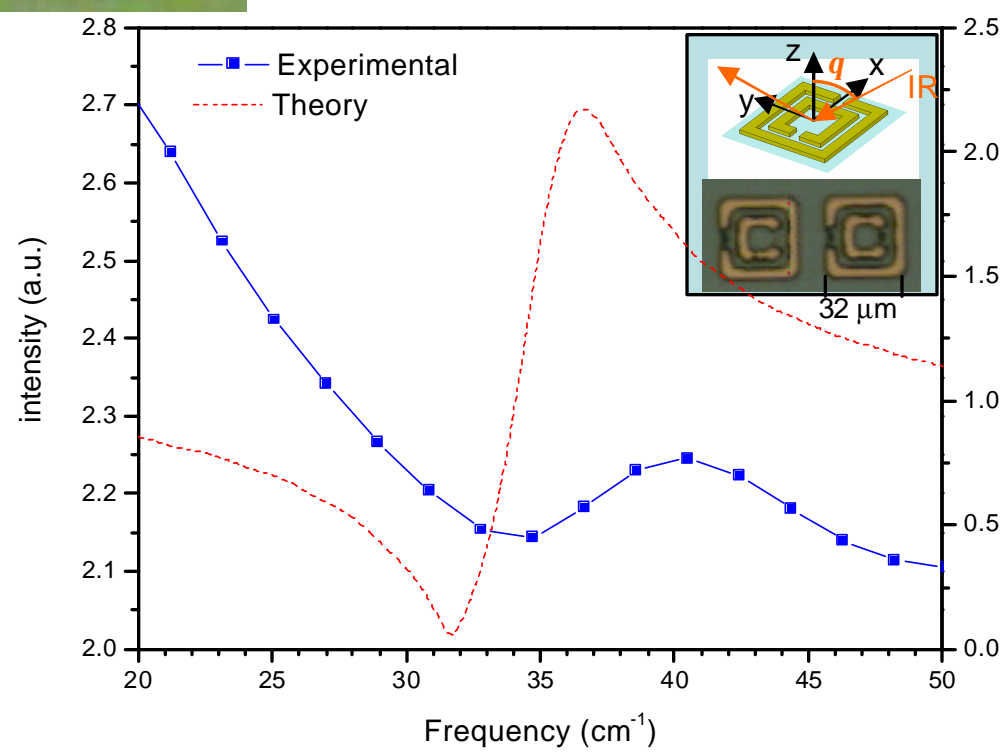




\title{
NanoARPES:A New Detector for nm-scale Electronic Structure Measurements
}

\author{
Eli Rotenberg* and Aaron Bostwick (LBNL, ALS) \\ *Principal Investigator
}

\section{Project description}

The goal of the nanoARPES is the development of a new probe for electronic structure measurements at the $50 \mathrm{~nm}$ or smaller length scale. The uniqueness of this probe arises from its ability to resolve the spectral function and the momenta of the electronic states, fundamental properties that cannot be determined by any other technique than Angle Resolved Photoemission Spectroscopy (ARPES). Determining these properties for nanometer devices is expected to have an impact on the fundamental understanding of correlated systems, quantum phase transitions, as well as practical problems in semiconductor physics, such as in solid-state lighting and solar power generation. But extension of conventional ARPES, in which a $\sim 50 \mu \mathrm{m}$ focused beam of soft x-rays induces photoelectron emission into an angle- and energy-resolving detector, to the nanometer scale requires novel solutions to problems on both the photon focusing and electron detection sides of the problem. In addition, demonstrations are needed to show the feasibility of the probe, and to show that potential obstacles such as space charge effect are not serious.

\section{Progress report}

The LDRD was initially aimed towards simulation and design of nanoARPES components. but the accomplishments during the reporting period exceeded the initial goals. Taken together, these accomplishments have demonstrated the feasibility and usefulness of the technique and have successfully generated a strong interest among a potentially large user base. We have also conducted the first (to our knowledge) sub- $\mu \mathrm{m}$ ARPES measurements. Specifically we have

1-conducted simulations on a novel electron collection optics, which is capable of high-angle-resolution detection over a large solid angle

2-conducted Monte-Carlo simulations of space charge effects in photoemission at the ALS to demonstrate the maximum energy broadening of $7 \mathrm{meV}$, within the design goal of $10 \mathrm{meV}$ resolution

3 -constructed a test-bed instrument capable with $300 \mathrm{~nm}$ spatial resolution for feasibility studies

4-demonstrated operation of prototype electron optics capable of detection over $12^{\circ} \times 12^{\circ}$ solid angle

5-successfully conducted measurements of the bandstructure of several important low-conductivity samples of fundamental interest

a) single crystal grains down to $2 \mu \mathrm{m}$ size from polycrystalline graphite

b) amourphous carbon grain boundaries in single-crystal natural graphite

c) $\mathrm{TaSe}_{2}$ microcrystals

d) $\mathrm{NbSe}_{3}$ quasi-1D wires down to $5 \mu \mathrm{m}$ width

6-measured the presence of chemically and electronically distinct domains of $\sim 5 \mu \mathrm{m}$ dimension in nominally homogenous manganites.

7-designed the next generation instrument capable of $50 \mathrm{~nm}$ resolution and temperatures down to $10 \mathrm{~K}$, and overcoming other limitations of the testbed system.

8-built a collaboration of outside scientists from academia and other institutions to join a nanoARPES collaborative 
Ultrafast Magnetization Dynamics

PIs: Andreas Scholl, Anthony T. Young, Jun Feng, Joachim Stöhr, Howard A. Padmore

\section{Project Description}

The purpose of this work is to develop techniques for the study of ultrafast dynamics in magnetic materials. On its own this is of forefront scientific interest, but it also offers a new and very exciting area for early exploitation on the ALS f-sec slicing source as well as future initiatives. The goal is to demonstrate that we can measure spin and orbital moments in magnetic systems as a function of time after laser or magnetic field excitation on a p-sec streak camera, and to investigate the range of scientific applications possible for such a revolutionary new tool.

X-ray pulses from the ALS are typically $80 \mathrm{psec}$ long, and so to achieve one p-sec time resolution, we will use streak camera detection. In order to measure and decode spin and orbital components of the magnetization, we will use the x-ray spectroscopic techniques of magnetic circular and linear dichroism. To achieve fast excitation, we require small excitation areas, and so we will use x-ray microfocusing techniques. Magnetic field excitation will use currents generated from laser driven GaAs current sources. A laser will also be used for direct electronic excitation. All of these techniques exist in some form at the ALS, and the approach here is to bring them together to attack this new area of $\mathrm{x}$-ray spectroscopy applied to psec magnetization dynamics. The team of PIs on this LDRD has expertise in all of the relevant areas.

\section{Accomplishments}

All components of the experiment: laser, optical setup, experiment chamber, and streak camera have been built, thoroughly tested, and used in demonstration experiments on $\mathrm{Ni}$ and GdFe. The experiments have confirmed the suitability of the chosen experimental approach. A time resolution of 900 fs was measured under optimal conditions using UV radiation in a multishot exposure. Using x-rays a resolution of about 2 ps was achieved. Improving this timeresolution down to a practical limit on the order of $100 \mathrm{fs}$ is an important goal for the next years.

Experiments on a Fe/Gd multilayer sample have demonstrated a fast and simultaneous demagnetization of $\mathrm{Fe}$ and $\mathrm{Gd}$ in response to an intense heating laser pulse. We observed that a complete demagnetization occurred within about 4 ps, and thus clearly resolved within the time resolution of the setup. This finding is contrary to observations using the optical Kerr effect, which indicated faster, sub-ps dynamics of the magnetization, albeit no complete demagnetization was achieved in most cases. The apparently slower transient dynamics probed by x-rays also appeared in measurements of Ni and FeNiPt samples. We are currently investigating whether this discrepancy is due to experimental limitations or an unambiguous proof that the ultrafast dynamics measured using light optics is not a true representation of the transient magnetization of the sample, which has been suggested by some. We were also able to determine the transient spin and orbital moment of Fe and Gd in order to study the transport of angular momentum between spin, electron and lattice degrees of freedom.

This first successful experiment demonstrated sufficiently high sensitivity to detect a fast magnetic response using x-rays to an ultrafast optical excitation. Additional experiment will be conducted to establish ultrafast x-ray detection as a primary tool for the study of ultrafast magnetism. We have started collaborations with groups at Hitachi HGST, SLAC Stanford, University Bochum, Max-Planck-Institut Stuttgart, and University Regensburg to develop the new capability towards becoming a user facility. 


\author{
High Average Power Laser Amplifier \\ Principal Investigators: Russell Wilcox and Robert Schoenlein
}

\title{
Project Description
}

The purpose of this project is to develop a Titanium:sapphire laser amplifier capable of providing tens of Watts average power at tens of $\mathrm{kHz}$ repetition rate, with high efficiency and excellent beam quality. Typically, repetition rates for high power amplifiers are much lower, which tends to reduce the effect of thermal lensing because of the larger beam size required for efficient energy extraction. In the high pulse rate case, a smaller beam size results in stronger thermal lens effects, requiring low temperature cryogenic cooling and careful optical design. Development of this amplifier will provide a compact source of moderate energy pulses at high repetition rate, enabling high data rate femtosecond optical experiments, including applications in synchrotron femtosecond x-ray pulse generation.

We will develop a regenerative pre-amplifier and a two-pass power amplifier based on cryogenically-cooled Ti:sapphire. The amplifiers will be designed to generate $\sim 60 \mathrm{~W}$ of average power at a repetition rate of $40 \mathrm{kHz}$, although lower rates are possible. Special cooling chambers for the 2-pass amplifier and regenerative amplifier will be designed, utilizing compact cryorefrigerators. Measurements will be made of the output power and energy, thermal lens focal length, and influence of the cryorefrigerator mechanical vibrations on the beam.

\section{Accomplishments}

We have built two state-of-the-art power amplifiers and tested them at a repetition rates of $10 \mathrm{kHz}$, with an incident average pump power of $90 \mathrm{~W}$, producing $2 \mathrm{~mJ}$ of energy per pulse, and $\sim 20 \mathrm{~W}$ average power in the amplified beam. The measuring cooling capacity of the cryorefrigerator and the absorption of the $10 \mathrm{kHz}$ pump demonstrates the feasibility of pumping at $40 \mathrm{kHz}$ with four pump lasers providing $90 \mathrm{~W}$ each, $10 \mathrm{kHz}$ (interleaved in time to generate the $40 \mathrm{kHz}$ ). The amplified beam quality has been measured, and the $\mathrm{M}^{2} \sim 1.1$ mode quality factor demonstrates that distortions of the beam from thermal lensing are negligible. Mechanical vibrations from the cryorefrigerators have been effectively mitigated.

We have also constructed a state-of-the-art cryogenically-cooled regenerative pre-amplifier operating at $10 \mathrm{kHz}$, and demonstrated $4 \mathrm{~W}$ output power in a nearly diffraction-limited beam. This pre-amplifier serves as the seed source for the two power amplifiers. We have design and constructed a novel passively-stabilized optical delay line. This line provides $\sim 300 \mathrm{~ns}$ of optical path delay with temporal stability of $<100 \mathrm{fs}$, and excellent beam pointing stability.

A novel pulse compressor based on holographic transmission grating has been designed and constructed. An overall efficiency of better than $75 \%$ over four grating transits has been demonstrated. This compact system allows efficient compression of the amplified pulses without significant distortion from thermal lensing at the grating surface. In addition, computer controlled optical transport lines for the laser system have been designed and are being implemented and tested. 


\section{Chemical Sciences Division}

LB04038

Gas phase studies of the building blocks of life

Principal Investigator: Musahid Ahmed

\section{Project Description}

The purpose of this project is to study the fundamental properties of molecules that are the building blocks of life. Dipole moments, ionization energies, electron affinities, proton affinities, molecular polarizabilities and electronic structure all contribute to the photochemistry, radiationless processes and reactivity necessary for biological functions. We will generate large biomolecules in the gas phase and perform time-of-flight mass spectrometry with tunable vacuum ultraviolet (VUV) light to measure these properties. Single photon ionization of these building blocks coupled to soft desorption techniques allow for generation of fragment free mass spectrum for large biomolecules. This opens up windows of opportunity in performing mass spectrometric imaging in real biological systems.

\section{Accomplishments}

Under the aegis of this program, we developed a new technique to introduce fragile biomolecules into the gas phase. The general strategy is to synthesize dry nanoparticles comprised of biomolecules that are then thermally vaporized in high vacuum. The resulting vapor, containing neutral biomolecules, can be "softly" ionized with tunable VUV synchrotron radiation producing nearly fragmentation-free mass spectra. In fact, the degree of fragmentation can be finely controlled via the thermal or photon energy, yielding greater insight into the role of internal energy in the photoionization mass spectra of amino acids and other bio-molecules. To first demonstrate the general utility of this approach and to illustrate how internal energy affects single photon ionization mass spectra, we examined the amino acid tryptophan in detail. In addition, the ionization energies of a number of biomolecules was measured; for some, these are the first reported measurements. Erythromycin (733 amu) is the largest molecule that has been detected completely fragment free with this technique thus far.

Very recently, new experiments were developed to allow VUV post-ionization of molecules laser desorbed from surfaces. Cholesterol, gramicidin D, erythromycin, tryptophan and triacontane surfaces were irradiated with a micron sized $527 \mathrm{~nm} 1 \mathrm{khz}$ laser beam. The resulting neutral plume was interrogated with VUV light and parent ions detected with a reflectron time of flight mass spectrometer. Delayed extraction indicated that fragmentation depends on the internal energy deposited in the molecule during the desorption process. In the near future, these experiments will incorporate ion beams in order to improve spatial resolution.

Laser desorption in conjunction with jet cooling allowed for the determination of the ionization energy of the DNA base guanine. These studies were extended to studying the effect of water on the ionization of histidine and cytosine by generating water clusters with these molecules in a supersonic expansion. The ionization energies for a number of cytosine-water and histidine-water clusters were measured.

Finally, we have measured the nano-phase ionization energies of glycine, phenyalanine-glycineglycine and Vitamin B12 by measuring a photoelectron spectrum using velocity map imaging. X-ray powder diffraction studies on the biological nanoparticles show that they are crystalline in nature and the reduced ionization energy when compared to the gas phase results suggests polarization effects play a role in the ionization process. The difference in the ionization energy between the gas and nano phase for glycine is the polarization energy and allowed for the first experimental determination of the molecular polarizability for glycine. 
Oxygen Catalysis through Proton and Electron Control: Biomimetic

Chemistry for Renewable Energy

Principal Investigator: Christopher J. Chang

\section{Project Description}

This project seeks to study the basic reaction types of renewable energy chemistry to provide a framework for developing sustainable, carbon-neutral energy cycles. Of particular interest are multielectron, multiproton reactions that underlie energy storage and release at oxygen-based feedstocks. Our synthetic designs are guided by principles of natural metalloenzymes that catalyze oxygen activation and evolution reactions during biological energy conversion. We seek to capture the functional essence of these enzymes and extend their chemistry beyond the constraints of the protein environment to provide pure synthetic constructs that control reactivity in the first and/or second coordination sphere. To meet the massive scales required for sustainable energy cycles and minimal environmental impact, new catalytic systems are being devised that rely on cheap and plentiful first-row transition metals like iron, manganese, and copper. Because formation and cleavage of oxygen-oxygen and oxygen-hydrogen bonds are predicated on dual electron and proton transport, research is being pursued on two parallel fronts: exploring new primary-sphere environments for controlling multielectron redox chemistry at first-row transition metal centers and installing second-sphere hydrogen-bond pendants to enhance reactivity at these primary synthetic active sites.

\section{Accomplishments}

Several new classes of soft-amide ligand frameworks have been developed for studying highvalent first-row transition metal chemistry. Primary-sphere geometries ranging from square planar to tetrahedral to trigonal mono/bipyramidal to octahedral are available using modular assembly of pyrrole, indole, carbazole, oxazoline, and peptide amide donors into various chelating structures. Carbazole-bridged iron and copper complexes are active catalysts for aziridination and cyclopropanation of simple olefins under mild conditions. In addition, amidebased iron templates are capable of activating hydrogen peroxide for olefin epoxidation, aryl C$\mathrm{H}$ oxidation, and oxygen evolution reactions.

Parallel efforts have explored the effects of second-sphere hydrogen bonding on smallmolecule reactivity at iron and manganese synthetic active sites. Acidic and basic pendants have been introduced, but no improvements in catalyst stability or reaction turnover have been observed yet. Current studies are aimed at adjusting the orientation between second-sphere pendants and first-sphere metal centers to tune reactivity. 


\section{Time-Resolved X-Ray Absorption Spectroscopy of Metalloporphyrins}

Principal Investigator(s): Stephen R. Leone

\section{Project Description}

This project is focused on the development of ultrafast X-ray transient absorption methods using table-top laser systems, with application to the direct probing of short-lived metal-centered excited states in metalloporphyrin and metal carbonyls. In these experiments, photoexcitation with femtosecond pulses in the ultraviolet to near-infrared results in the formation of electronically excited states in the molecules. Ultrashort x-ray pulses will then probe the subsequent time-evolution of these excited states along the relaxation pathway to the ground state. With this technique, we expect to observe processes occurring on timescales of tens of femtoseconds, thereby providing x-ray probing of these ultrafast processes with unprecedented time resolution.

A table-top amplified Ti:sapphire femtosecond laser system is used to produce high-order harmonics in a capillary waveguide filled with a rare gas. The harmonic soft x-rays are passed through the sample, along with a pulsed optical pump beam. The pump pulse triggers a photoprocess and the high-order harmonics probe the changes in the transient absorption of the metal center. The harmonic output is spectrally dispersed with a grating after the sample to preserve the best possible time resolution. The dispersed light is detected in differential transient absorption by a soft x-ray CCD camera to obtain a spectrum of the metal center absorption as a function of time during the metal-ligand charge transfer or dissociation.

\section{Accomplishments}

With funding from the FY2005 LDRD, we have designed and assembled the high vacuum apparatus required for the experiment. Equipment funds obtained in FY2005 have also allowed us to purchase a new laser system that yields $>2.5 \mathrm{~mJ}$ pulse energy and sub-35-fs pulse duration at $1 \mathrm{kHz}$ repetition rate. High harmonics have been generated in a $150-\mu \mathrm{m}$ internal diameter capillary waveguide filled with argon and detected using both a XUV photodiode and the custom-built soft $\mathrm{x}$-ray spectrometer. At input pulse energies as low as $0.35 \mathrm{~mJ}, \sim 10^{8}$ photons per pulse per harmonic are obtained at $\sim 30 \mathrm{eV}$. Increasing the input pulse energy to $\sim 2 \mathrm{~mJ}$ resulted in extension of the harmonic emission in argon to the aluminum $\mathrm{L}^{\mathrm{II} / \mathrm{III}}$-edge at $\sim 72 \mathrm{eV}$. The photon energy of the harmonics generated so far $(\sim 30-70 \mathrm{eV})$ spans the spectral range for the $\mathrm{M}^{\mathrm{II} / \mathrm{III}}$ edge transitions in first row transition metals, thereby enabling us to investigate metal-centered excited states in metal carbonyls and metalloporphyrins.

A setup for single filamentation of the amplified output from the Ti:sapphire laser system has been implemented to obtain ultrashort pump pulses in the near-infrared by the method of pulse spectral broadening and recompression. Pulse spectra obtained so far using this technique have yielded spectral bandwidths that can support $\sim 7$-fs pulses. To access visible radiation for eventual experiments on metalloporphyrins, a visible noncollinear optical parametric amplifier (NOPA) with $\sim 140 \mathrm{THz}$ spectral bandwidth has also been assembled. 
Spectroscopy and Dynamics of Pure and Doped Helium Nanodroplets Principal Investigator: Daniel M. Neumark

\section{Project Description:}

This project will perform photoionization and photoelectron spectroscopy experiments on atoms and molecules absorbed onto large helium droplets, $\sim 10^{4}$ atoms, in order to understand how these fundamental and well understood gas phase processes are altered in the environment of a helium droplet. A helium droplet source will be constructed and used on Endstation 3 of the Chemical Dynamics Beamline at the Advanced Light Source (ALS). Photoionization mass spectrometry and photoelectron spectroscopy experiments will be performed using the high-throughput vacuum ultraviolet monochromater on this endstation.

\section{Accomplishments:}

During the past year we have carried out photoionization and photoelectron spectroscopy experiments on $\mathrm{He}$ droplets doped with $\mathrm{SF}_{6}$ and rare gas atoms. These experiments are carried out on the Chemical Dynamics Beamline at the Advanced Light Source. Three types of experiments were performed: photoionization mass spectroscopy, in which the photon mass spectrum is measured using a newly implemented time-of-flight mass spectrometer configuration on the beamline, photoion imaging, in which the photoion velocity distribution is determined for ions formed in droplets, and photoelectron imaging, in which the effect of the droplet on photoelectron energy and angular distributions is determined. The most extensive work was performed on droplets doped with $\mathrm{SF}_{6}$. $\mathrm{The} \mathrm{SF}_{6}{ }^{+}$ion formed by photoionization undergoes dissociation with $100 \%$ yield, primarily to $\mathrm{SF}_{5}{ }^{+}+\mathrm{F}$. Photoion imaging reveals that the $\mathrm{SF}_{5}^{+}$product is slowed down considerably when the $\mathrm{SF}_{6}$ is photoionized in a droplet, as compared to photoionization of the bare molecule. The photoelectron images of bare and clustered $\mathrm{SF}_{6}$ are quite similar, showing that this effect is not due to the initial ionization process but instead arises from interaction of the $\mathrm{SF}_{5}{ }^{+}$with the He atoms as it is ejected from the droplet. We find that the $\mathrm{SF}_{5}{ }^{+}$velocity distribution from doped droplets can be simulated by a simple binary collision model. 


\section{Advanced Computational Methods for Photon- Molecule Collision Processes Principal Investigators: Thomas N. Rescigno and C. William McCurdy}

\section{Project Description}

Sophisticated calculations of collisional ionization processes involving two electrons in the continuum have so far been limited to light atomic targets. For analogous processes involving simple molecular targets, no first-principles calculations have appeared to date. The purpose of this project is to develop advanced computational methods for studying certain photon-molecule collision processes that are currently beyond the grasp of first principles methods. We will develop grid-based ab initio approaches, based on the method of exterior complex scaling (ECS), for studying double photoionization (one photon in, two electrons out) of small molecules. These theoretical methods will assist in the interpretation of current experiments at the ALS on double photoionization of $\mathrm{D}_{2}$ and provide a predictive tool for motivating new experiments.

\section{Accomplishments}

For our initial efforts on molecular double photoionization, we capitalized on the work that had gone into developing the atomic ECS/B-spline approach and developed an analogous approach for molecules based on single-center expansions. This effort produced the first completely $a b$ initio calculation of singly differential cross sections for double photionization of $\mathrm{H}_{2}$ and have now been extended to produce triply differential cross sections (TDCS) for oriented target molecules that can be compared directly with the ALS experiments. Although the calculations are far from complete, our initial results, which have been accepted for publication in Science, have already begun to reveal the wealth of complexity contained in the experimental data and have confirmed the speculation that the pattern of electron ejection, which depends sensitively on bond distance, is a tool for measuring changes in molecular electron correlation. Our calculations have revealed that even in the COLTRIMS experiments done at the ALS, the finite range of acceptance angles necessarily used in analyzing the data may dramatically affect the interpretation of the TDCS. A definitive set of completely converged calculations, carried out for different values of the target internuclear distance, will be necessary to completely explain these beautiful experiments.

We have demonstrated, for the simplest molecule, that problems in which two unbound electrons escape the target can be attacked successfully by combining grid methods with single-center expansions. This approach, however, is difficult to extend to more complex molecular targets since the presence of singular nuclear attraction potentials separated by more than a Bohr radius causes slow convergence of the single-center expansion. In the region near the nuclei, decades of experience in quantum chemistry have shown that basis set methods are optimum. We are therefore combining the two approaches in a hybrid method that uses Gaussian basis sets centered about the nuclei and grid-based finite elements in an exterior-scaled discrete variable representation in the asymptotic regions of space. The basic viability of the method, which we described in a Physical Review A publication, was demonstrated on one-electron problems and we are very close to having a two-electron code for studying molecular hydrogen that can be tested against our current single-center codes. The result should be a computationally efficient method that eliminates the problem of slow convergence associated with single-center expansions. But more importantly, the combination of Gaussians with grid-based methods will enable us to use molecular orbitals to describe inner-shell electrons and still treat two active electrons in the continuum.

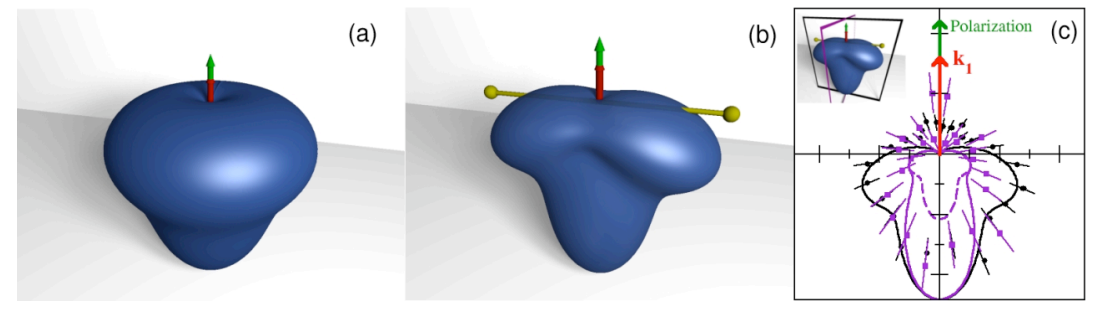

Comparison of triple differential cross sections for double photoionization of helium (a) and $\mathrm{H}_{2}$ (b) for a case where one ejected electron is fixed along the direction of photon polarization and the other is varied. Comparison with ALS experiment shown in (c). 
A New Approach for the Catalytic Conversion of Methane and Other Inert Hydrocarbons Principal Investigator(s): T. Don Tilley

\section{Project Description}

The selective, catalytic functionalization of saturated hydrocarbons represents one of the most important challenges in chemical research. While some progress has been made, there are very few processes which allow conversion of the cheapest and most abundant hydrocarbon, methane. Intense interest in this topic has led to many important advances including the discovery of several mechanisms by which transition metal species react with unactivated $\mathrm{C}-\mathrm{H}$ bonds. While these studies have revealed a number of interesting stoichiometric transformations, there have been significantly fewer reports describing selective conversions of alkanes via homogeneous catalysis. Methane is a particularly attractive substrate for such conversions since it is cheap and readily available, and represents a potentially useful reagent for the incorporation of methyl groups into molecular structures.

For some years, we have been studying $\sigma$-bond metathesis (the concerted exchange of substituents between two $\sigma$-bonds) as a fundamental process for bond activations, in the context of metal-mediated polymerization chemistry. Studies on the interactions of silanes with $\mathrm{d}^{0}$ metal complexes have revealed several pathways for the activation of $\mathrm{Si}-\mathrm{H}$ and $\mathrm{Si}-\mathrm{C}$ bonds via $\sigma$-bond metathesis. This rich reaction chemistry suggested that similar activation steps might be used in catalytic hydrocarbon functionalizations, given appropriately active and selective catalysts. In preliminary tests of this concept, we have observed the first catalytic processes that allow conversion of methane to other products via $\sigma$-bond metathesis with $\mathrm{Cp}_{2}{ }_{2} \mathrm{ScMe}\left(\mathrm{Cp}^{*}=\mathrm{C}_{5} \mathrm{Me}_{5}\right)$ as catalyst. These observations raise the exciting possibility for development of homogeneous catalytic processes that utilize methane as a cheap and convenient methylating reagent. We therefore plan to pursue this possibility in the development of new catalytic conversions for methane and other inert hydrocarbons.

\section{Accomplishments}

Efforts in this program have centered on exploration of systems related to the scandium one described above, to learn as much as possible concerning the influence of ancillary ligands, reaction conditions, and substrate variations. It is clear that a soft, strongly donating ligand (e.g., $\mathrm{Cp}^{*}$ ) supports $\sigma$-bond metathesis reactivity, and variations in the structure of such ligands should reveal factors that influence reactivity in this chemistry. Since electron-rich, anionic $\pi$-systems are generally good candidates, we have developed routes to permethylindenyl $\left(\mathrm{C}_{9} \mathrm{Me}_{7}{ }_{7}, \mathrm{Ind}^{*}\right)$ complexes of yttrium, and these are currently being examined as $\sigma$-bond metathesis catalysts. A related ligand that has potential for promoting $\sigma$-bond metathesis reactivity and selectivity in bond activations (due to its steric bulk) is permethylfluorenyl $\left(\mathrm{C}_{13} \mathrm{Me}_{9}{ }^{-}\right.$, Flu*). Nonamethylfluorene is a new compound which has recently been synthesized in our laboratory. This compound should exhibit a high basicity, and we expect it to be more strongly donating than $\mathrm{C}_{5} \mathrm{Me}_{5}{ }^{-}$. We are therefore investigating the coordination chemistry of the Flu* ligand, with group 3, lanthanide and early transition metals. Investigations of complexes of zirconium and ruthenium indicate that the Flu* ligand produces highly electron rich metal centers.

A parallel effort involves investigation of scandium and yttrium complexes containing ligands derived from the linking of two cyclopentadiene-like groups. Such ansa-type ligands are known to generally increase metal reactivity, and we would like to define the influence of this effect on $\sigma$-bond metathesis chemistry for scandium and related metals. These investigations began with an ansa ligand that is closely related to the $\mathrm{Cp}_{2}{ }_{2}$ ligand set, $\left[\mathrm{Me}_{2} \mathrm{Si}\left(\mathrm{C}_{5} \mathrm{Me}_{4}\right)_{2}\right]^{2-}$. Scandium complexes of this ligand have been prepared, some of which activate methane. Interestingly, $\left[\mathrm{Me}_{2} \mathrm{Si}\left(\mathrm{C}_{5} \mathrm{Me}_{4}\right)_{2}\right] \mathrm{ScMe}$ is not a catalyst for the addition of methane across the double bond of propene (as is $\mathrm{Cp}_{2}{ }_{2} \mathrm{ScMe}$ ), but with its more "open" structure it catalyzes the corresponding reaction involving the more hindered olefin isobutene. 


\title{
Computing Sciences
}

LB05008

\author{
Cryptographic Foundations for New Generation Distributed Systems \\ Principal Investigator: Olivier Chevassut, Abdelilah Essiari
}

\section{Project Description:}

New generation distributed systems (e.g., peer-to-peer and Grid middleware) are networking technologies that enable complex interactions among computational and data resources. If these new systems are to be successful and widely deployed in production computing environments, they need to be enhanced with proper security mechanisms. Current security technologies cannot support the complex message exchange models (e.g., one-way, asynchronous, bilateral, multi-node paths) used by these state-of-the-art middleware. While the previous security notions are still valid, the message exchange models require new cryptographic foundations.

The present project lays down the security foundations to design and develop an essential component of next generation distributed systems. The project first produces provably-secure cryptographic algorithms such as (one-time) password-based authentication and key exchange for two or more parties. Next, it implements a prototype that will support the complex message exchange models needed by next generation distributed systems. Finally, it makes sure that these algorithms retain their "provablesecure" features in practice by specifying implementation guidelines.

Accomplishments:

Our most significant accomplishment has been to design provably-secure cryptographic algorithms for next generation distributed systems. These algorithms adapt the Diffie-Hellman key exchange and encrypt the message flows using a password. We have shown how to securely encrypt using either a password-keyed symmetric cipher or a mask generation function, and how to prove, through very "elegant" and compact proofs, that the algorithms are secure under reasonable mathematical assumptions. These algorithms are authentication methods for two-party or multi-parties, and will play an essential role in securing next generations distributed architectures.

We have developed prototype implementations based on these password-based authentication algorithms. The first prototype provides the establishment of a security context between parties at the application level (i.e. using Web Services primitives) while the second prototype does it at the transport level. The prototypes exchange a master secret key using the password-authentication algorithm, derive session keys from this master secret using a key-derivation technique, and ensures message data integrity using a symmetric cipher.

We are in the process of providing detailed specifications of the implementations-which can be used as evidence of the correctness of the prototypes--and of providing guidelines for the use of our cryptographic algorithms to make sure that they retain their provable-secure characteristics in practice. 


\section{Interactive Visualization Methods for Exploration and Comparison of Multi-billion Base Pair Sequence Data \\ Principal Investigator: Bernd Hamann}

\section{Project Description}

This project supported efforts concerning visualization-based analysis of sequence data and highresolution imaging data. Another project component was concerned with improvements of visualizationbased techniques for protein structure prediction.

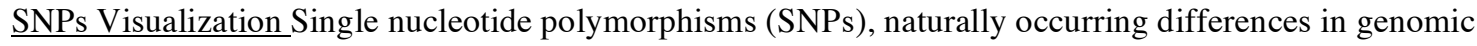
DNA sequences inside a population, have been used extensively to study the evolution of microbial populations. The recent application of random "shotgun" sequencing to environmental samples make possible more extensive SNP analysis of co-occurring and co-evolving microbial populations. An intriguing finding from the Tyson et al. study was the mosaic nature of the genomes of an archaeal population inferred to be the result of extensive homologous recombination of three ancestral strains. This observation was based on a manual analysis of a small subset of the data (ca. 40,000 basepairs) and remains to be verified across the whole genome. Tools to analyze this type of data are in their infancy. We have developed a tool called "SNP-VISTA," a publicly available interactive visualization tool that assists scientists in the analysis of ecogenomics data for studying homologous recombination in microbial populations.

3D Gene Expression Visualization Animal embryos comprise dynamic 3D arrays of cells that express gene products in intricate spatial and temporal patterns that determine the shape and form of the developing animal. Biologists have typically analyzed gene expression and morphology by visual inspection of photomicrographic images. A rigorous understanding of developmental processes, however, requires methods that can quantitatively analyze these phenomenally complex arrays with cellular resolution. As part of the Berkeley Drosophila Transcription Network Project (BDTNP) we have developed a variety of interactive visualization tools to address this issue, and to analyze and find patterns in high-resolution imaging data characterizing gene expression .

Energy Visualization for Protein Structure Prediction Many ab-initio methods for protein structure prediction are based on minimization of energy functions of candidate protein conformations. We have developed a system to visualize the make-up of these energy functions by breaking down their overall values into spatially separated components associated with individual atoms, bonds, atom triples, and dihedrals. Visualizing the constituent terms together with the 3D protein conformation gives additional insight into the behavior of the optimization process.

\section{Accomplishments}

We have developed an interactive visualization tool, "EcoSNP-VISTA" that provides a visual interface for semi-automatic analysis of SNPs data from ecogenomics data. A compact color-coded representation of SNPs data allows a scientist to manually detect recombination points and visually verify automatically calculated recombination points. EcoSNP-VISTA provides insight into homologous recombination in microbial populations and has the potential to guide in the development of computational methods for such analysis.

Further, we have adapted novel volume-rendering methods to multi-channel confocal microscopy data. Every single channel of an image stack is rendered independently by mapping brightness information to color and transparency information. Resulting colors are blended using user-specified weights, supporting seamless blending between channels. We have also combined our volume visualization prototype has been combined with user interaction tools to support quantitative determination of the accuracy of the BDTNP's nuclear segmentation methods. Nuclei are rendered to include information obtained from a nuclear segmentation mask. This work has already yielded significant improvements in segmentation accuracy.

We also have developed a visualization tool for $3 \mathrm{D}$ gene expression data in the form of matrices that describe nuclear positions and their associated gene expression levels. Our current prototype system combines multiple views in physical space and gene expression space.

For protein structure prediction applications, we have created an energy visualization tool to work in combination with the "ProteinShop" protein modeling software. This tool uses volume rendering to visualize the makeup of a protein's internal energy as colored "clouds" of energy surrounding less optimized parts of a protein structure, and allows a user to direct the optimization algorithm towards these regions. 


\section{Peer-to-Peer Resource Discovery Framework \\ Principal Investigator: Wolfgang Hoschek}

\section{Project Description:}

For large distributed multi-disciplinary science collaborations it is a difficult problem to find data, results, and resources. Typically, defining centralized storage and managing the resources as a single domain solves this problem. But this solution scales poorly and does not allow for opportunistic use of resources and data repositories. As a much more scalable alternative, we in this project research, design, develop, evaluate and benchmark a reusable software infrastructure for Peer-to-Peer resource discovery, thereby enabling a range of innovative research directions building on it. The infrastructure addresses a number of scalability problems in a general way. It provides flexible and uniform transport-independent resource discovery mechanisms to reduce both the client and network burden in multi-hop P2P systems.

We in this project develop a flexible, powerful and extensible resource discovery and data integration framework. This framework provides a common resource discovery framework for a wide variety of scientific applications. It is built around an applicationlevel messaging protocol we are designing and building called Peer-to-Peer I/O (P2PIO). The abstractions that P2PIO can support are quite powerful. The work completed on this project includes the research, design, development, evaluation and benchmarking of a plug-in based reusable software infrastructure for Peer-to-Peer resource discovery, thereby enabling a range of innovative research directions building on it.

Accomplishments:

In this project we have developed a new model and software (firefish) for resource discovery in decentralized Grid systems. This model is derived from our work in developing Grid Database Frameworks for use by scientists to support large-scale Grid projects. The multi-purpose $\mathrm{P} 2 \mathrm{P}$ resource discovery infrastructure model we developed supports a wide range of use cases with a small set of extensible building blocks. The protocol is query language independent and XML data model independent. It supports pull and push based data delivery, and can accommodate static as well as dynamic data sources, while being agnostic with respect to transport, network, and topology.

It is essential that the P2PIO is efficient so in this project we also designed and developed a novel general-purpose binary XML encoding for network messages (bnux) that is faithful to XML, self-contained, tunable for performance or size and verification of wellformedness. To verify the performance of the library, we have conducted a detailed quantitative evaluation that contrasts tree and streaming deserialization/serialization as well as compression efficiency of this and other binary schemes with best-of-breed production-quality XML toolkits, each of them carefully applied in optimal configuration.

The Firefish and bnux libraries developed during this project have been documented and released via the web at http://www.dsd.lbl.gov/software.html. 
Optimizing Genomic Data Storage for Wide Accessibility

Principal Investigators: Nancy Meyer, Jonathan Carter, Damian Hazen

\section{Project Description}

The purpose of this project is to distribute, enhance access to and archive the data generated at the DOE Joint Genome Institute's Production Genome Facility making this large collection of genomic data publicly available. The DNA sequence is considered the fundamental building block for the rapidly expanding field of genomics. These sequences are used in parallel by the whole genomics community to help identify genes and regulatory elements, understand evolution and study differences between individuals. The PGF has a constantly increasing DNA sequencing capability and requires a scalable reliable solution for the projected data flows of billions of files and terabytes of data per year. The solution features long term archiving, nearline availability and practical access solutions for millions of tiny files. The JGI produces between two and four million files per month and the facility is expanding. A methodology is needed that would scale with the number of files and the amount of data.

\section{Accomplishments}

We deployed a new software interface to the archival storage system for this project. Htar, written by Michael Gleicher, can be used to organize data into meaningful collections and store these collections as one file in the archive. Htar maximizes data transfer using buffering techniques and builds an "Htar" file in the storage system by transferring the individual files over the network requiring no extra space in the local file system. Once the "Htar" file is created in the storage system it moves as a single file through the archive. Access to the data in the archive over time, as it migrates to dense media, is now scalable: one access brings the whole collection online. This solution can change according to the type and amount of data making it expandable and configurable. Collections can be organized and sized for maximum access. The solution is scalable; millions of files can be accessed as one file, and terabytes of data can be sized appropriately for access: for instance, as a one-terabyte file or many four-gigabyte files, depending on the expected usage of the data.

The first "data" to flow to NERSC was the raw data produced at PGF used to generate sequences. JGI is currently archiving 80 gigabytes of raw data every night and the data management database at JGI is updated with the location of the raw data in the NERSC archive. The raw data at NERSC has been recalled many times to JGI to reproduce sequences that were incorrectly generated. In one case, PGF staff were able to retrieve six months of raw data and reliably reprocess it in forty-five days.

Now that an archival methodology is in place at JGI, it could be used to enhance access to the genomic data for the scientific community by archiving more types of genomic data and enhancing JGI's Web site to directly access archival data from NERSC as well as local JGI data. The archive could also be used to improve the reliable sequencing of data at JGI by using the currently-stored raw data to test new software or systems as they are installed at JGI. 


\section{Parallel Methods for Robust Optimization and Uncertainty Quantification Principal Investigator(s): Juan C. Meza}

\section{Project Description}

Optimization of functions derived from the modeling and simulation of a physical process constitutes an important class of problems in many scientific applications. Often, the computer simulation entails the solution of a system of nonlinear partial differential equations (PDE) in two or three dimensions. Other applications include particle dynamics simulations, problems in chemical kinetics, and PDE constrained optimization problems. The main characteristic of these types of problems is that the function evaluation is computationally expensive and dominates the total cost of the optimization problem. Depending on the nature of the application and the solution method employed, there can also be noise associated with the evaluation of the objective function and the experimental data. In many applications it is also the case that there are uncertainties in both the model parameters and the experimental data. In these cases it is desirable that the optimal solution be robust in the sense that small changes in the model parameters or data do not generate large perturbations in the optimal solution. This research focuses on two major areas: 1) the development of parallel algorithms for robust optimization under uncertainty and 2) the development of methods for simulation-based optimization.

\section{Accomplishments}

Our main accomplishments have been the development and application of optimization methods for several new scientific problem areas. The first application involved the development of a new methodology for protein structure prediction that not only allows the user to create initial configurations based on known protein fragments but also allows them to steer the global optimization solver. The method uses a combination of global optimizations in subspaces of the dihedral angles space and local optimizations in the full dimensional space to find the protein configuration with the lowest energy. A crucial component of the protein prediction methodology is an accurate energy model that discriminates good folds from misfolds. An important accomplishment of this effort has been the upgrade of our energy code to be consistent with AMBER7 with modified parameters and the coupling of that code with another LBNL developed code called ProteinShop. The modified parameters are designed to improve the discriminatory ability of the energy function by enforcing the formation of hydrogen bonds and beta-sheets. We have tested our method during the most recent CASP6 worldwide competition for the assessment of protein structure prediction methods. Furthermore, the coupling of the new energy model with ProteinShop has led to the development of an energy visualization feature, which permits us to highlight regions of high energy to guide the steering process.

Another important accomplishment has been the integration of a separate optimization software package, NOMAD (developed by Mark Abramson), for the optimization of mixed variable problems to a simulation code, SYNOW, that is used to compute the spectra of supernova. Using this combination, we have been able to estimate the parameters of SYNOW so that the computed synthetic spectra match known spectra from supernova. This was the first time that this process has been automated and holds the promise of being able to quickly detect type Ia supernovae from experimental data. The preliminary results are highly encouraging and the application of a new parallel optimization method is currently underway. 


\author{
Enhancing Commodity Scalar Processors with Vector Components \\ for Increased Scientific Productivity \\ Principal Investigators: Leonid Oliker, John Shalf, Katherine Yelick, William T. Kramer
}

\title{
Project Description
}

As we enter the era of billion transistor chips, computer architects face significant challenges in effectively harnessing the large amount of computational potential available in modern CMOS technology. We are increasingly constrained by the comparatively lower rate of improvement for off-chip connections. The increasing gap between processor and memory speeds is also a well-known problem in computer architecture. Furthermore, techniques designed to hide memory latencies, such as out-of-order superscalar instruction processing, speculative execution, multithreading, and stream prefetching engines, may actually increase the memory bandwidth requirements. This so-called "memory wall" is one of the primary reasons many high performance applications run well below the peak arithmetic performance of the underlying machine. In particular, irregularly structured and data-intensive codes exhibit poor temporal locality and receive little benefit from the automatically managed caches of conventional microarchitectures. In addition, a significant fraction of scientific codes are characterized by predictable data-parallelism that could be exploited at compile time with properly structured program semantics; supercalar processors can often exploit this parallelism, but their generality leads to high costs in chip area and power, which in turn limit the degree of parallelism.

The goal of our research is to understand the impact and opportunities for improved scientific productivity by combining vector processing features with commodity processors. In particular, we will identify the minimum set of hardware features required to see the benefits of vectorization without compromising the cost-effectiveness of commodity processors for general purpose processing. We also plan to explore alternative caching strategies, such as software controlled memory architectures, due to their potential of being programmed to emulate the behavior of vector registers.

\section{Accomplishments}

Our initial effort has focused on exploring vector extensions to microprocessor ISAs, establishing a VIVA-2 collaboration with IBM, obtaining and extending the IBM Mambo simulator to simulate VIVA-2 (or vector extensions) on the Power processor, exploring state-of-the-art approaches to software controlled memories, and a preliminary investigation of the CELL architecture that offers a testbed for evaluating the potential advantages of using software controlled memories for scientific applications.

We examined the forthcoming STI Cell processor as a building block for future high-end computing systems. Our work contains several novel contributions. We are the first to present quantitative Cell performance data on scientific kernels and show direct comparisons against leading superscalar, VLIW, and vector architectures. We developed both analytical models and simulators to predict kernel performance. Our work explores the complexity of mapping several important scientific algorithms onto the Cell's unique architecture. Additionally, we propose modest microarchitectural modifications that could significantly increase the efficiency of double-precision calculations.

Our preliminary investigation has also identified several opportunities for potential architectural modifications. The first change required to support vector execution is to include a Vector Length register that allows programs to specify the number of operations each vector instruction performs. Secondly, vector memory instructions need to be added to allow programs that exhibit strided or indexed memory access patterns to be vectorized. We have worked closely with IBM Research, adding extensions to their Mambo PowerPC full-system simulator that will allow us to simulate vector extensions on commodity processors, to address prefetch and strided memory access limitations of modern microprocessors. 


\title{
Advanced Computational Tools for Electric Power Systems
}

\author{
Ali Pinar, Bernard Lesieutre, Chao Yang, Juan Meza, Vanessa Lopez, Vaibhav Donde
}

\section{Project Description:}

As the society's dependence on engineered networks such as the electric power and communication networks expand, the need for secure and reliable operational standards for these systems becomes vital for the economic, energy and national security of our country. The August 14, 2003 blackout in the Northeast depicted the possible catastrophic consequences of a few broken power lines. The need for improved operational and reliability standards for the electric power system is incontestable. Such efforts however, are hindered by the lack of computational tools that can provide better modeling capability and system understanding. Our research will enable improved reliability and operational standards for electric power systems by developing computational tools for better system modeling.

We claim improved reliability and operational standards will come with advanced computational tools that give better modeling capability to engineers. With a team of laboratory experts from Computational Research Division and Environmental Energy Technologies Division, we have been investigating the computational challenges in electric power systems with emphasis on security of the power grid.

\section{Accomplishments:}

The vulnerability of a power network is traditionally studied by what is called " $n-k$ " security analysis. Given a network with $n$ generators and transmission lines, one examines the consequences of any $k$-component failure by removing associated components from the original system. The exhaustive enumeration of all possible scenarios becomes prohibitively expensive when $k$ is larger than 2, and efficient methods that can detect vulnerabilities without exhaustive enumeration need to be designed.

In the first year of the project, we have focused on mathematical modeling of the power system vulnerability analysis. In our formulation, we measure the distance between the current operating point and the feasibility hypersurface, where the distance is measured in units of broken transmission lines. Moreover, we have designed methods to measure the severity of a blackout, since we are mostly interested in significant blackouts that affect a large area, as opposed to a trivial outage that affects a single house.

Measuring the distance from the current operating point to the feasibility hypersurface requires solving a nonlinear optimization problem. We have successfully applied our model to detect vulnerabilities of a typical power network, by using existing software to solve the associated nonlinear optimization problem. Our early results prove the validity of our models, but further algorithmic research is required to solve the nonlinear optimization problem. We are currently working on improved algorithms that can better exploit the characteristics for our nonlinear optimization problem. 


\section{Statistical Feature Modeling for Scientific Data Via Basis Decomposition Principal Investigator: Raquel Romano}

\section{Project Description}

This project explores statistical approaches to feature extraction for multidimensional data, focusing on basis decomposition methods such as independent component analysis (ICA) for computing data-driven representations of scientific data. Many current scientific data analysis methods have been specifically designed for a particular data set and/or experimental setting and hence are not easily adaptable to inevitable changes in data acquisition and experimental goals. Furthermore, in many cases the phenomena of interest are not well understood beforehand, so precise criteria for feature extraction are not explicitly defined. Statistical criteria are used to directly infer a basis representing interesting features in a data set; for example, the application of ICA to imagery yields localized, oriented spatial filter kernels that represent edge features. Thus, without designing a filter bank to explicitly search for known features, ICA derives a basis that may be used to represent salient features in the data. The goal of this project is to develop statistical algorithms for feature extraction, anomaly detection, and exploratory analysis of spatial and spatiotemporal scientific data sets well-suited for statistical feature analysis, namely a) the quantification of protein signals in microscopy imagery, b) the detection of resonances in particle trajectories and grid potentials in accelerator simulations, and c) the detection of tropical cyclones in high-resolution climate simulations. These goals will be pursued with the aim of implementing analysis tools that interface naturally with existing visualization platforms built and maintained by the LBNL Visualization Group, so that application scientists may use analysis results in conjunction with data exploration and visualization.

\section{Accomplishments}

In the first year of this project, we have explored both supervised and unsupervised methods for extracting image features corresponding to the presence of DNA-repair proteins in large sets of multi-channel microscopy images of irradiated cells. In response to the growing need for flexible, computational methods for quantifying subcellular phenomena in imagery, we explored three approaches to learning and image: 1) the Support Vector Machine (SVM) model for allowing users to train a feature detector to accept or reject candidate protein signals, 2) the Gabor function wavelet basis for multi-scale and multi-orientation feature representation, and 3) independent component analysis (ICA) for building a data-driven basis for separating salient features from background noise in large cellular image sets. These methods have been implemented as modules in FMAT (Fluorescence Microscopy Analysis Tool), a GUIbased image analysis tool we built for LBNL life scientists to analyze cell images either interactively or in batch mode. The core FMAT application segments cell nuclei in a single channel to provide cell boundaries for further subcellular analysis, on top of which we have built prototype analysis components for quantifying subcellular protein signals. Our application of ICA to these images captures the salient protein signals without requiring threshold-based image processing routines. We continue to explore this approach to analyzing both image data and higher-dimensional scientific data. 


\author{
Evaluation of Computer Architecture Alternatives \\ Principal Investigators: Paul Hargrove and Katherine Yelick
}

\title{
Project Description
}

The goal of our study is to evaluate the technology building blocks of high performance machines for future acquisitions at LBNL, and across DOE, by examining both established and emerging technologies for processors, memory systems and interconnects from industry, academia and government laboratories. This work provides the DOE with insight into emerging designs and their potential effects on next-generation supercomputing architectures and the applications that run on them. Additionally, it provides a mechanism for evaluating future designs through collaboration with industry, and thus has the potential to influence performanceenhancing system features in those designs.

Our approach uses a combination of microbenchmarks, application kernel benchmarks, and performance models to measure the benefits and limitations of emerging architectural technologies. The application kernel benchmarks help identify the need for new algorithms for a given architecture, and allows us to explore an entire class of problems. The microbenchmarks isolate particular aspects of the hardware that prove to be a bottleneck, and thereby enable studies of variations on a particular hardware implementation. Finally, our study investigates performance models to predict the behavior characteristics of next-generation architectures.

\section{Accomplishments}

We developed and utilized a parameterized benchmark called Stencil Probe, which emulates grid-based codes, to identify memory system bottlenecks on current microprocessors and to measure the effectiveness of cache optimizations. Our study determined that cache tiling techniques designed for 2D grids that were effective in the past are less effective today because of increasing cache size, the trend towards 3D computations, and the importance of prefetching. Cache tiling techniques decrease cache misses, but often increase the rate of non-contiguous memory accesses. We quantified this trade-off by developing a simpler Stanza Triad benchmark, which measures the effectiveness of prefetching on unit and non-unit stride accesses. From these results, we constructed a memory model based on the importance of prefetching to predict performance of cache-blocked stencil codes on architectures with given cache sizes.

To gain insight on the direction of future interconnect design, we evaluated several largescale scientific codes using the IPM library to record runtime communication characteristics, including the types and sizes of communication in each application. Our work demonstrated that most applications communicate with a relatively small number of partners, suggesting that fullyconnected interconnects are over-provisioned for most scientific codes. In addition, we showed that most global communication occurs using small message sizes, rarely utilizing the available bandwidth. Using these insight, we explored the feasibility of a hybrid interconnect that uses passive/circuit switches to dynamically provision active/packet switch blocks at runtime. Our work shows that such an interconnect topology would be suitable for a large class of DOE scientific codes.

Lastly, we have participated in numerous discussions with HPC vendors, informing them of our application requirements, and suggesting design choices that would improve performance of DOE codes on future architectural designs. 


\section{Earth Sciences Division}

LB03008

\section{REAL-TIME PCR WITH REVERSE TRANSCRIPTION FOR QUANTIFICATION OF CHLORINATED SOLVENT DEGRADATION \\ Lisa Alvarez-Cohen and Terry Hazen}

\section{RESEARCH OBJECTIVES}

The purpose of this project was to develop and apply culture-independent molecular approaches for characterizing microbial communities capable of degrading chlorinated solvents in subsurface aquifers. Our approach was to apply quantitative real-time PCR (qPCR) and reverse transcription to quantify the occurrence and expression of dehalogenating species and genes in laboratory enrichments under varying conditions. Whole-genome microarrays were also applied to query the transcriptome of Dehalococcoides strains.

\section{ACCOMPLISHMENTS}

\section{Functional-gene expression analysis using quantitative PCR}

By combining qPCR with reverse transcription, we can accurately monitor the expression level, defined as the ratio of mRNA to DNA, of any expressed gene. We have used this technique to study changes in the expression of three Dehalococcoides functional reductase genes: tce $A, v c r A$ and $b v c A$, within pure cultures of Dehalococcoides as well as in a variety of mixed communities and in field samples. We found that expression of all three genes rose dramatically after cells were exposed to sovents that they are active on, but not after exposure to other solvents. E.g., an expression response was observed for the tceA gene after exposure to TCE or isomers of DCE, but not PCE or VC. Similar results were observed for the $v c r A$ and $b v c$ genes. Further, detecting the presence of specific functional genes with qPCR was demonstrated to be an effective mechanism for identifying and differentiating specific strains of Dehalococcoides within mixed communities. This technique was used to evaluate the optimal enrichment solvent for generating microbial consortia capable of degrading TCE completely to ethane.

\section{Analyzing genomics and transcriptomics using whole-genome microarrays}

An Affymetrix whole-genome microarray for D. ethenogenes 195 was designed in collaboration with Gary Andersen of LBNL and Professor Stephen Zinder of Cornell University. The queried transcriptome of strain 195 under optimal growing conditions produced statistically significant hybridization signal on roughly $95 \%$ of the 1,600 -gene chip. In contrast, the transcriptome of a related strain, BAV1, exhibited $40 \%$ hybridization. The reproducibility between replicates and TCE and PCE exposed cells was excellent, with $\mathrm{R}^{2}$ values above 0.99 . 


\title{
Scientific Basis for Advanced Geologic Storage Technologies
}

\author{
Principal Investigator: Sally M. Benson
}

\section{Project Description}

The purpose of this project is to develop the scientific basis for the next generation of technology for geologic storage of carbon dioxide in deep underground formations. Today, geologic storage technology relies primarily on physical trapping beneath a low permeability caprock to keep $\mathrm{CO}_{2}$ trapped securely underground. Over longer timescales, on the order of hundreds to thousands of years, the $\mathrm{CO}_{2}$ dissolves into the formation fluids and eventually can form carbonate minerals. Once dissolved or mineralized, $\mathrm{CO}_{2}$ is in essence, stored permanently. The purpose of this project is to identify physical, chemical and biogeochemical approaches that can be used to accelerate the formation of permanently stored or immobilized $\mathrm{CO}_{2}$. This project will identify a set of options for accelerating dissolution and mineralization of $\mathrm{CO}_{2}$ and rank them with regard to state of scientific knowledge, potential for rapidly accelerating these reactions, and technical feasibility. Approaches with the highest potential will be selected for systematic analysis, including numerical simulation and laboratory experimentation.

\section{Accomplishments}

This year we have selected two important mechanisms for additional investigation: capillary trapping and dissolution of $\mathrm{CO}_{2}$ into formation brines. Capillary trapping occurs as the storage formation rewets with brine. As the brine invades the pore spaces, the $\mathrm{CO}_{2}$ plume forms disconnected bubbles that can not migrate through the pore throats. Dissolution of $\mathrm{CO}_{2}$ into brine effectively immobilizes $\mathrm{CO}_{2}$ by trapping it in brines that have hundreds of thousands to millions of years residence times in deep basins. The short and long term effectiveness of these mechanisms depends on the small-scale structure of the rocks, which determine the capillary pressure and relative permeability to $\mathrm{CO}_{2}$ and brine. While these concepts are well established for oil, water and methane mixtures, there are no published studies specifically for $\mathrm{CO}_{2}$-brine systems. In addition, long term behavior of capillary trapping, where dissolution is also occurring has not been studied.

We have carried out laboratory experiments of $\mathrm{CO}_{2}$ displacing brine at the pore-scale $(1 \mu \mathrm{m})$ at the microtomography beamline at the ALS and core scale (10's of cm) at our CT scanning facility. These data provide information on the relative permeability and capillary pressure of typical formation rocks and how they relate to pore-structure. Unusually shaped relative permeability curves appear to be caused by the very large influence of small scale heterogeneity on displacement of brine by $\mathrm{CO}_{2}$. These observations probably explain why displacement of brines by $\mathrm{CO}_{2}$ is relatively inefficient. On the other hand, low displacement efficiency is helpful for accelerating dissolution into brines. Implications for capillary trapping are not yet certain. 


\section{Autonomous Sensors for Ocean Dissolved Organic Matter \\ Principal Investigators James Bishop, Paul Luke}

\section{Project Description}

Dissolved organic carbon (DOC) in seawater constitutes a large, dynamic, yet poorly quantified reservoir of carbon in the oceans. Changes in the concentration of DOC reflect transformation of carbon between inorganic and organic, and between dissolved and particulate species. DOC changes may thus be a critical yet independent measure of changes in the biological pump in the ocean or of changing carbon sequestration in the ocean. DOC presently must be measured in shore based laboratories on ship collected water samples.

Here, we propose development of a novel autonomous ocean-profiling sensor for real time detection of dissolved organic carbon in seawater. Such a sensor when combined with other LBNL pioneered sensors will allow -- for the first time -- fully autonomous quantification of all products of marine photosynthesis on the very fast time scales of marine photosynthesis. Our effort supports current national initiatives for carbon prediction, management, and assessment.

Marine biofouling begins with adsorption of an organic film on a fresh surface. The process initiates on timescales of seconds. Our sensor concept is to present to the environment a pre-cleaned organic-free surface purposefully engineered for non-specific adsorption of dissolved organic matter from seawater. The developing organic film will be monitored for seconds, the surface will be cleaned and the measurement process repeated. This multi-year LDRD project aims to explore mass and electrochemical approaches to the measurement of DOC and to prove that we can achieve a repeatable and robust sensor suitable for use in the ocean.

\section{Accomplishments}

In FY04 the project used a thermal and pressure stabilized platinized Quartz Crystal Microbalance (QCM) to conduct adsorption/desorption experiments in flowing solutions of sucrose $\left(\mathrm{C}_{12} \mathrm{H}_{22} \mathrm{O}_{11}\right)$ in buffered $0.1 \mathrm{M} \mathrm{NaCl}$ at seawater $\mathrm{pH}$ (near 8). The detection limit (6000 $\mu \mathrm{M} \mathrm{C}$ ) was at least 3 orders of magnitude above that required to measure DOC at oceanic levels (40-300 $\mu \mathrm{M} \mathrm{C}$ ) at the required $0.5 \mu \mathrm{M}$ C precision. The QCM sensor was anything but stable.

This year (FY05) we constructed a new apparatus to measure changes of surface (doublelayer) capacitance of an electrode array due to organic adsorption reactions. The new apparatus allowed improved investigation of electrochemical cleaning. Our batch addition experiments using artificial seawater at $\mathrm{pH} 13$ (adjusted using $\mathrm{NaOH}$ ) demonstrated detection limits of 6 and $0.5 \mu \mathrm{M}$ C for sucrose and natural DOC (supplied by Dr. D Repetta, at Woods Hole Oceanographic Institution), respectively. We further determined that the current apparatus is not obviously sensitive to fluctuations of temperature and pressure in the laboratory and thus is much more suitable for adaptation for in-situ operation in seawater.

Slow drift (over hours) in the baseline presently makes it difficult to determine the absolute DOC concentration without a suitable reference or ability to add or remove DOC. We are working systematically to improve the stability of the apparatus. Drift effects can be eliminated as an effective surface cleaning method is achieved. A high priority of our work is DOC detection (and cleaning) at neutral $\mathrm{pH}$.

In summary, work during FY05 demonstrated that the capacitance measurement approach can achieve adequate sensitivity for detection of oceanic DOC at natural concentration levels. There is significant work remaining to do during FY06 to meet project goals. 
Development of Biosensors for Endocrine Disrupting Compounds in Agricultural Watersheds Sharon Borglin, Chris Campbell, Will Stringfellow, Bailey Green, Eleanor Wozei, \& Allen Grayson

\section{Project Description}

While water recycling (treatment and reuse of wastewater) is an important strategy for reducing water scarcity, concerns about residual contaminants in the recycled water remains a limiting barrier to wide spread implementation. For example, Endocrine Disrupting Compounds (EDCs) in recycled water may occur at concentrations that can mimic hormones involved many biological processes including immune function, reproduction, growth, and control of other hormones. EDCs are hormonally active at small concentrations (parts per billion or trillion). The list of EDCs found in water is steadily growing and includes many common agricultural, industrial and household chemicals and their degradation products. Significant sources of EDCs include both synthetic chemicals like pesticides and those produced naturally by plants and animals.

There are two major obstacles limiting the successful management and removal of EDCs in the environment: (1) EDCs are a diverse group of compounds present and active in very low concentrations and (2) EDC sources are numerous and poorly characterized. In response, we developed analytical capabilities measure specific EDCs in urban and agricultural sources and to develop biosensors to detect overall EDC activity. The goals of this project were to develop novel analytical techniques for the measurement of target EDC compounds, to develop biosensors for measurement of overall EDC activity that could be adapted to field use for real time monitoring, and to develop a prototype system for field deployment.

\section{Accomplishments}

Background research produced an extensive literature review of available biosensors for monitoring the fate and transport of EDCs in water which was submitted for publication. Extensive bioassay development was completed on time-lapsed EDC activity response in live estrogen-sensitive yeast cells using fluorescence. This fluorescence assay was used in conjunction with Fourier-Transform Infrared (FTIR) spectromicroscopy, allowing the collection of real-time data on response of live cells to estrogenic compounds to determine maximum estrogenic response of the assay which is crucial for accurate and consistent determination of overall estrogenicity. These bioassays were used to monitor estrogenic activity in water samples and the response was studied using microscopy and spectroscopy to evaluate cell structural changes and viability. Traditional analytical techniques (GC, GC/MS and HPLC) and ELISA kits were used for calibration and verification of bioassays and for analysis of specific estrogenic chemicals in water, wastewater, wastewater solids, and sediment. In addition, the prototype field biosensor system was developed for deployment of fluorescent assays and was successfully tested with model organisms and compounds in the laboratory.

These analytical techniques were applied to samples collected from three waste water treatment systems. Analysis of select EDC compounds, 17-b- estradiol and testosterone, generally showed $40-60 \%$ removal during treatment, and removal was dependent both on the type of treatment process and the time of year. The fluorescent and yeast based assays, which measure total estrogenicity, showed a similar decrease in activity during treatment, but also showed increasing estrogencity during some stages of the processes, demonstrating that there are degradation products causing an estrogenic response and that analysis of a select group of compounds may be insufficient to determine if treatment is successful prior to releasing effluent. 


\title{
Micro-characterization and chemical micro-dynamics of atmospheric mineral dust
}

\author{
PI: Donald J. DePaolo
}

\section{Project Description}

The objective is develop isotopic analysis and modeling techniques to better characterize the sources and transport of atmospheric mineral dust and the chemical and physical transformations that affect its properties. Mineral dust is an important component of the atmospheric aerosol budget. It is now recognized that dust travels long distances in the atmosphere, even to the point that a substantial fraction of the dust present over the western U.S. comes from Asia. Dust therefore constitutes a means of intercontinental material exchange and has important health and security implications. Dust also affects the atmospheric radiation balance and therefore is a critical component of the global warming issue. Our objective is to evaluate the extent to which isotopic techniques can be used to fingerprint dust sources and evaluate how these techniques can be best applied. A critical aspect of this is sampling techniques. Another aspect is microchemical analysis and imaging. A third component of the study is the development of Lattice-Boltzmann codes for modeling the micro- physics and chemistry of dust grain surfaces. This project combines lab capabilities in isotopic geochemistry, synchrotronbased analysis and imaging, and computational physics and chemistry.

\section{Accomplishments}

In the first year of the project we have been able to begin tests of the characterization methods. We have analyzed a time series of Teflon PM2.5 filters from Heifei, China, taken in Spring 2002 during a dust storm that eventually carried dust to the western U.S. So far we have successfully made isotopic measurements of $\mathrm{Sr}$ and determined that in most cases there is sufficient $\mathrm{Sr}, \mathrm{Nd}$ and $\mathrm{Pb}$ on the filters to allow for time-series analysis of the isotope ratios, combined with Xray analysis of chemical constituents and imaging. We have made progress on new mass spectrometric techniques that should allow isotopic measurement of $\mathrm{Nd}, \mathrm{Pb}$, and possibly $\mathrm{U}$. We continue to develop sample analysis protocols, including filter blanks and serial leaching and dissolution. NCEM facilities are being used to evaluate effects of sample leaching as well as to characterize the chemical and morphological characteristics of the particulates. A new method of sulfate isotopic analysis is being developed that will allow us to measure $\mathrm{S}$ and $\mathrm{O}$ isotopes in particulates. We have established a collaborative working relationship with researchers at U.C. Davis and the California Air Resources Board to further develop sampling protocols.

As a first step toward simulating dust-surface reactions we have adapted an existing 2D Lattice Boltzmann code for the simulation of microscale kinetics of crystal growth from a fluid medium. This code has been modified to handle multiple reacting species and we have applied it to the problem of mass-dependent isotopic fractionation associated with water vapor deposition on ice crystals in clouds. The code allows us to relate for the first time vapor phase saturation, crystal growth rate, crystal morphology (fractal dimension), and isotopic fractionation due to equilibrium partitioning and vapor phase transport. 


\title{
The Behavior and Impact of Nanoparticles in the Environment
}

\author{
Principal Investigator: Benjamin Gilbert
}

\section{Project Description}

Natural nanoparticles are virtually ubiquitous products of inorganic precipitation and weathering, as well as both sources and products of microbial metabolism. They participate in numerous biogeochemical processes but their impact depends factors such as redox behavior and transport properties that are generally not well characterized. Research in the following areas is particularly relevant for understanding the fate of nanoparticles in the environment, and for beneficial applications of nanoscience in the application and monitoring of remediation technologies: 1. The transport of natural and anthropogenic oxide nanoparticles; 2 . the stimulation of bioremediation with ferric iron nanoparticle amendments; and 3. the determination of basic materials properties of nanoparticles, particularly for calibrating methods of field-scale monitoring of nanoparticle precipitation.

\section{Accomplishments}

The transport of iron oxide nanoparticles in aqueous systems is crucially affected by their tendency to aggregate. Consequently, we are studying the colloid properties of $6 \mathrm{~nm}$ diameter iron oxyhydroxide nanoparticles as a model for numerous natural and man-made oxide nanoparticles. Using light and x-ray scattering, we showed that repulsive Coulombic interparticle interactions stabilize nanoparticle dispersions without aggregation, as expected from classical theories of colloid stability. However, we additionally observed the formation of stable, finite size clusters of nanoparticles even under conditions in which macroscopic aggregation does not occur. This unexpected result shows that nanoparticle transport models must be developed that describe the hydrodynamic behavior of such submicron nanoporous clusters. This research was the basis of a recent successful funding application to the DOE BES program (in collaboration with Guopeng Lu, LBNL).

The amendment of water soluble ferric iron nanoparticles into contaminated sub-surface zones may provide a novel method for stimulating the bioremediation of heavy metal and organic contaminants. As described above, uncoated iron oxide nanoparticles remain dispersed in solution only in a narrow range of $\mathrm{pH}$ not commonly found in nature. Therefore, we have developed a method to bind organic ligands to the surface of $6-50 \mathrm{~nm}$ diameter goethite nanoparticles in a post-synthesis step. The ligands are chosen both to solubilize the nanoparticles in circumneutral conditions, as well act as the organic substrate for FeRB metabolism. Thus, it is hoped that a single economical amendment will stimulate bioremediation activity without either iron or carbon limitations on microbial growth. Laboratory experiments are planned to test the growth of Geobacter using coated goethite nanoparticles as the sole energy source.

Finally, we have completed an extensive experimental investigation on the mechanical properties of synthetic and biogenic $\mathrm{ZnS}$ nanoparticles. We studied synthetic $\mathrm{ZnS}$ nanoparticles of different sizes, crystal phase, and crystallinity, plus one sample of biogenic $\mathrm{ZnS}$ nanoparticles extracted from a column of sulfate reducing bacteria. The $6 \mathrm{~nm}$ diameter nanoparticles exhibited the same properties as bulk $\mathrm{ZnS}$. However, for a particle diameter of $\sim 3 \mathrm{~nm}$ (the typical size of biologically produced environmental nanoparticles) all nanoparticles studied were considerably more compressible than bulk $\mathrm{ZnS}$. These studies will provide quantitative input for modeling the acoustic wave detection of nanoparticle precipitation. 


\title{
The California Water and Energy System: An Approach for Addressing Future Crises
}

\author{
Principal Investigators: Norman L. Miller, Nigel T.W. Quinn, Larry L. Dale, Helen He \\ Collaborators: CA Depart. Water Resources, U.S. Bureau of Reclamation, and National Weather Service
}

\section{Project Description}

The overall purpose is to understand how natural processes and human intervention interact to influence water supply and water quality, and the sensitivity of the system to potential disruptions. We are developing tools that provide reliable quantitative evaluation of risks under different future climate scenarios, and integrating physical, water quality and allocation modeling to quantify water supply reliability under changing conditions. Our goals are to: (1) Quantitatively understand the climate drivers impacting mountain front recharge, snowmelt runoff, and net infiltration in the Sierra Nevada and Central Valley. (2) Investigate the sensitivity of a series of simulated droughts and the response of water table response and to pumping withdrawals, and if a new equilibrium state or unsustainable conditions are the outcome. (3) Adapt existing geophysical logging and monitoring techniques as a superior method for characterizing the depth distribution of groundwater contaminants. (4) Develop a regional resource management model to demonstrate economic tradeoffs between agricultural and environmental groundwater pumping, incorporating long-term aquifer degradation. (5) Test electricity data to better characterize groundwater pumping in the Central Valley, and develop a model to better forecast electricity and groundwater use.

Two spatial scales are considered to understand detailed physical processes and policy implications. These scales are the State level (California), and the Local level (Transect: Merced River Basin west to the adjacent Grasslands Ecological Area). At present, there is no research group that brings together these core components.

\section{Accomplishments}

Our FY05 accomplishments include coupling, testing, and application studies of the NCAR Community Land Surface Model version 3 (CLM3) with the LBNL groundwater code, TOUGH2. We have further advanced our 100-year baseline climate and drought-perturbed climate for testing the groundwater recovery rates at the Merced Basin-Grasslands Ecological Area, and have begun a more detailed examination of drought response for the entire Central Valley using the CDWR Central Valley Groundwater Surface-water Model version 2 (CVGSM 2). This latter effort is viewed as high impact and is expected to result in significant findings on drought response that will be part of the State Water Plan. Progress on the Merced and Westside groundwater surface-water model continues, as does the subsurface characterization in collaboration with Montgomery Watson-Harza, who performed geophysical logging at Grassland Ecological Area and deployed a Cone Penetrometer Transducer to work in parallel with the LBNL field testing.

Additionally, we have collected baseline water supply and use data for agricultural and urban districts in the Central Valley and other regions of California, formulated an electricity groundwater-pumping model, calibrated the model using regional water-source data, and obtained preliminary estimates of historical groundwater pumping for selected regions in the Central Valley. A conceptual model of joint reservoir and aquifer management has been designed to integrate this information and describe the tradeoff between regional demands for electricity and water. The conceptual model suggests variables for evaluating gains from joint reservoir aquifer management, agricultural water conservation and conjunctive use programs. More detailed modeling will allow the authors to quantify potential gains from joint reservoir-aquifer storage management. 


\section{Next-Generation Codes for Modeling Subsurface Processes}

PIs: George Moridis, Stefan Finsterle, and Eric Sonnenthal

\section{Project Description}

The objective of this work is the development of a new generation of codes to extend, couple, streamline, and unify the processes and capabilities currently implemented in the TOUGH family of multiphase flow. The increased complexity of environmental and energy resources problems indicates a need to refine hydrodynamic property descriptions and to incorporate new, relevant processes that affect the fate of fluids and contaminants. Moreover, the code should be designed for easy incorporation of geophysical modeling capabilities to enable forward and inverse modeling studies designed for characterizing heterogeneity and for reducing model structure errors.

In order to achieve these scientific goals and to preserve world-wide acceptance of TOUGH in geothermal, environmental, hydrocarbon resource recovery, radionuclide transport, and $\mathrm{CO}_{2}$ sequestration applications, the next generation of codes for modeling coupled subsurface processes must be based on a solid, transparent, and uniform computing platform with a flexible data structure, modular architecture, and coherent implementation of features based on established coding conventions and well-defined interfaces. The objective of re-engineering TOUGH is to ensure a framework within which the new capabilities can be developed and maintained in a safer, more efficient, traceable, and transparent manner.

\section{Accomplishments}

In FY2005, the functional requirements - as identified in FY2004—were implemented into the new-generation code (called temporarily TOUGH-Fx). The code architecture has been adapted to take advantage of the object-oriented concepts of data encapsulation, inheritance, and polymorphism provided by FORTRAN95. The new structure has significantly increased the code's transparency and traceability. Furthermore, code maintenance and the addition of complex modules with a large number of possible phase combinations (such as in TOUGH$\mathrm{Fx} /$ Hydrate) has been made much more efficient. Significant progress has been made in the coding of TOUGH-Fx, specifically (1) memory for large arrays is now dynamically allocated; (2) new gridding capabilities were added, allowing the easy description of irregular domains and heterogeneous spatial distributions of geological media properties; (3) enhanced capabilities were added for the description of heterogeneous spatial distribution of initial conditions; (4) thermodynamic properties of water have been expanded from sub-freezing to super-critical conditions, and related phase changes have been implemented; (5) equations-of-state describing the behavior of an expanded list of fluids of interest to subsurface science haven been developed (e.g., real gas mixtures of alkanes, $\mathrm{CO}_{2}, \mathrm{H}_{2} \mathrm{~S}, \mathrm{H}_{2}$, etc.); (6) an equation-of-state module for gas hydrates (TOUGH-Fx/Hydrate) has been finalized; (7) forward calculation of geophysical data has been implemented and coupled to the time-dependent hydrological system state for subsequent joint inversion of hydrological and geophysical data; and (8) work on a new user interface with flexible, high-level input language has begun. In addition to the core module of TOUGH-Fx, five equation-of-state modules are currently available in the new TOUGH-Fx environment, namely: TOUGH-Fx/Hydrate, /Air $-\mathrm{H}_{2} \mathrm{O}, / \mathrm{Gas}-\mathrm{H}_{2} \mathrm{O}$, /VOC, and /Richards. Training courses for TOUGH-Fx/Hydrate were held. The reengineering and development work of TOUGH-Fx is being documented in developers and users manuals. 


\section{Advancing the Next Generation of Rock-Fluid Imaging and Stimulation Technologies \\ Principal Investigators: Garrison Sposito, Kurt Nihei, Steve Pride, Ernest Majer}

\section{Project Description}

Increasing production in oil recovery operations and enhancing the removal of contaminants from groundwater aquifers are major goals in petroleum and environmental engineering, respectively. Seismic wave stimulation is emerging as an important technology for improving efficiency in achieving these goals because it is both economical and ecologically clean, and it overcomes some of the limitations of existing enhanced oil recovery methods. A central issue in developing seismic wave stimulation as a mature, reliable field technique, however, is determining the fundamental physical mechanisms by which seismic waves mobilize pore fluids in subsurface reservoirs.

In this project, we are developing a systematic, comprehensive continuum-mixtures approach based on partial differential equations in order to quantify the mechanisms responsible for the stimulation effect and to describe it numerically. The major goal is to provide a solid theoretical basis in continuum physics to advance the next generation of subsurface imaging methods and fluid stimulation technologies used for hydrocarbon recovery and groundwater remediation.

\section{Accomplishments}

During the first year of the LDRD project, we formulated a boundary value problem for investigating the dynamic response of a water-saturated porous medium to fluid pressure pulsing at seismic frequencies. In order to determine exact analytical solutions of the boundary value problem, a choice of normal coordinates that makes the problem completely separable into partial differential equations representing independent modes of wave motion is highly desirable. In the second year of the project, we developed an analytical theory for low-frequency behavior of dilatational waves propagating through saturated and partially-saturated porous media. At sufficiently low frequencies, the governing partial differential equations decouple into a propagating wave equation and a dissipative wave equation (telegraph equation).

We extended our decoupling procedure to a homogeneous elastic porous medium containing two immiscible fluids. The theory is based on the Berryman-Thigpen-Chin model, in which changes in capillary pressure between the two fluids are neglected. We show that lowfrequency dilatational wave behavior in partially-saturated porous media must be formulated in terms of a single hypothetical fluid having average or effective properties of the two fluids. The stipulation of "low frequency" underlying the derivation of our equations requires that the excitation frequency of wave motions be much smaller than a critical frequency equal to the inverse of an intrinsic time scale that depends on the kinematic viscosity and effective permeability of each fluid. Numerical calculations indicate that the critical frequency in both unconsolidated and consolidated materials containing water and a nonaqueous phase liquid ranges from $\mathrm{kHz}$ to $\mathrm{MHz}$. Thus many engineering problems involving the dynamic response of a partially-saturated porous medium to low excitation frequencies (e.g. seismic wave stimulation) should be accurately modeled by our equations after suitable initial and boundary conditions are imposed. 


\section{Biogeochemical Reaction Rates and Pathways in Porous Media \\ Principal Investigator: Carl Steefel}

\section{Project Description}

The purpose of this project is to dramatically increase our understanding of the scale dependence of biogeochemical and geochemical reaction kinetics in natural porous media. A second goal is to quantify the rates of precipitation and nucleation of important secondary mineral phases in soils and aquifers, a critical deficiency in current hydrogeochemical models. The present lack of understanding limits our ability to develop effective bioremediation schemes for contamination cleanup, to develop predictive models for $\mathrm{CO}_{2}$ sequestration in deep aquifers, and even to determine the fundamental controls on the rates of chemical weathering, an important long-term regulator of atmospheric $\mathrm{CO}_{2}$ levels.

The research approach is to collect baseline reaction rate data using conventional well-mixed flowthrough reactors and less conventional microfluidic reactive flow devices and then use these rates to interpret averaged or effective rates in porous media. As model systems, we are considering (1) the dissolution of calcite (a ubiquitous subsurface phase), (2) the abiotic and microbially-mediated reductive dissolution of Fe-hydroxides (important phases in bioremediation and natural attenuation of contaminants), (3) the dissolution of olivine (a model system with relevance to the problem of $\mathrm{CO}_{2}$ sequestration), and (4) clay formation at circumneutral $\mathrm{pH}$.

A variety of transport regimes ranging from pure diffusion to advection-dominant will be considered in the porous media flow transport experiments. X-ray synchrotron microtomography of the pore structure of the porous media, along with other spectroscopic and microscopic tools for mineral characterization (Scanning Transmission X-Ray Microscopy or STXM, and micro$\mathrm{X}$-ray diffraction or $\mu$-XRD), will be used to develop a basis for understanding how the bulk "averaged" rates in the porous media experiments relate to rates determined in the absence of "porous media effects" (i.e., using conventional well-stirred flowthrough reactors).

\section{Accomplishments}

A major accomplishment of this project is to have carried out the world's first microfluidic reactive flow experiments involving geologically-relevant materials. The first experiments involved the mineral calcite, within which a $20 \mu \mathrm{m}$ wide and $40 \mu \mathrm{m}$ high channel was etched using a femto-second laser. Even given the short residence time in the channel (30 seconds), the extent of reaction was enough to raise the $\mathrm{pH}$ of the injection fluid from 5 to 7.5 over the 2 centimeter length of the pore. Despite the substantial reaction in the channel, the rates of calcite dissolution were approximately two orders of magnitude slower than those determined in wellstirred flowthrough reactors, indicating the importance of pore-scale concentration gradients on the bulk rates of reaction. A microfluidic reactive flow experiment involving the reductive dissolution of Fe-hydroxide is planned for January and will involve in situ imaging of reactive phases using STXM at the Advanced Light Source.

A second significant accomplishment is the first measurement of clay (kaolinite) precipitation rates at ambient temperature and pressure $\left(25^{\circ} \mathrm{C}\right.$ and $\left.1 \mathrm{bar}\right)$. Initial experiments indicate that the kaolinite dissolution and precipitation reactions are reversible, although there is some suggestion from the data that the "steady-state" rates are time-dependent. The experiments also allow the quantification of the dependence of the rates on $\Delta \mathrm{G}$, the Gibbs free energy of reaction. Future dissolution-precipitation experiments will be conducted at $\mathrm{pH}$ values of 6 and 8 . 


\section{Engineering Division}

LB05010

Novel Imaging Detectors for Materials and Biology Research

Principal Investigator(s): P. Denes, H. Padmore

\section{Project Description}

The purpose of this project is lay the groundwork for a new program in imaging detectors. LBNL's long experience in specialized detectors for particle physics and astronomy provide the technical foundation, and this project includes specific R\&D to realize high-impact, new types of detectors. We are focusing on improving the ubiquitous detector in synchrotron radiation research and electron microscopy, based on a scintillating phosphor fiber-coupled to a CCD. In order to improve sensitivity and live time, as well as enabling new kinds of dynamic experiments, we are developing a highspeed CCD which can be read out at the rate of $5 \mathrm{~ms}$ per megapixel while maintaining a dynamic range in excess of 14 bits. In addition, we are developing an innovative electron microscopy detector, which will not only be fast and sensitive, but provide excellent spatial resolution - particularly at the higher energies which are of interest for thick biological specimens.

\section{Accomplishments}

In the past year we have concentrated on the design of these two detectors. For the fast CCD, we have performed simulation studies and optimizations, and have prepared a software framework which allows the automated layout of CCDs. Thick, fully depleted CCDs, which were developed by LBNL, have several interesting properties, and the work of this project has added new performance capabilities. In addition, we have made the first direct $\mathrm{x}$-ray measurements with a thick CCD.

For the electron microscope detectors, we have made measurements and taken first images on prototype structures, and have designed and fabricated two medium-scale detector prototypes, one optimized for very high sensitivity and the other high speed insitu materials studies. As part of the design process, extensive electron trajectory simulations have been performed, and initial results confirm the excellent point spread function which can be obtained with this new detector. 


\title{
Environmental Energy Technologies Division
}

LB05012

\author{
Constraining Ammonia Emission Inventories for Control of Air Quality \\ Principal Investigator: Marc L. Fischer
}

\begin{abstract}
Project Description:
Quantifying ammonia $\left(\mathrm{NH}_{3}\right)$ fluxes between the land surface and atmosphere is required for effective control of aerosol concentrations affecting human health, regional climate, and visibility. Ammonia $\left(\mathrm{NH}_{3}\right)$ is emitted in large but uncertain amounts from animal agriculture, and in lesser amounts from imperfect use of nitrogen fertilizers in crop agriculture, from catalytic converters used on automobiles, and from other energy related industrial processes. In areas where $\mathrm{NH}_{3}$ is limiting aerosol concentrations, controls being contemplated on combustion related emissions of $\mathrm{NO}_{\mathrm{x}}$ and perhaps $\mathrm{SO}_{\mathrm{x}}$ will not be effective in controlling aerosol. Hence, measurement of $\mathrm{NH}_{3}$ flux is essential for development of scientifically sound aerosol control strategies, and for improving nitrogen use in agricultural practices.
\end{abstract}

Improving $\mathrm{NH}_{3}$ emission inventories requires sensitive flux measurements (fluxes 1-10 umol m $\mathrm{m} \mathrm{hr}^{-1}$ ) over a wide range of meteorological conditions. The most direct and robust method for making flux measurements is eddy covariance (EC). EC measurements of $\mathrm{NH}_{3}$ flux have not been possible because previous instruments were not sufficiently sensitive at the frequencies required to capture turbulent transport in the surface layer $(>1$ $\mathrm{Hz}$ ). In this project we will develop a new instrument to accomplish sensitive flux and concentration measurements by combining a fast response $\mathrm{NH}_{3}$ sampling system with a compact quantum cascade laser (QCL) spectrometer and supporting micrometeorological instruments. Using the instrument we will measure measure $\mathrm{NH}_{3}$ emissions from different agriculture settings, and to examine the role of $\mathrm{NH}_{3}$ concentrations in aerosol formation.

Accomplishments:

In FY 2005, we completed development of the field instrument including incorporation of the QCL spectrometer and the fast-response $\mathrm{NH}_{3}$ sampling and calibration system into a field-packaged system. In fall, 2005, we successfully completed initial field testing. Results from these tests demonstrate that the instrument achieves the necessary fast response and sensitivity to allow eddy covariance flux measurements of $\mathrm{NH}_{3}$. We are now planning field campaigns: First, we plan to participate in the joint NSF, NASA, and DOE Megacity Study in Mexico City in March, 2006. We are also currently planning a measurement campaign to investigate aerosol formation in the Sierra Nevada with the California Air Resources Board for later in 2006. 


\section{Highly Efficient PLED Through Polymer Morphology Control}

PIs. Nitash Balsara, Jean Frechet, Steve Johnson, John Kerr, Gao Liu \& Rachel Segalman

\section{Project Description}

A major barrier to the development of high efficiency PLEDs (Polymer Light Emitting Diodes) for solid-state lighting applications is the efficient conversion of the both the singlet and triplet exciton energy to visible radiation. It has been demonstrated that phosphor dopants mixed into small molecule OLED devices are able to efficiently interact with the excitons to produce visible radiation. As a corollary to the small molecule OLED work, the goal of this institutional LDRD is to use polymer design, synthesis and morphological control to achieve high internal quantum efficiencies in a polymer-phosphor PLED.

We propose to incorporate a phosphor into a block copolymer system and use selfassembly to fabricate high efficiency, easy to manufacture PLEDs. In this triblock copolymer system, the dye dopants will be the middle block, where electron and hole transport blocks are attached at opposite ends of the molecule. The triblock copolymers will be designed to microphase separate into regular, nanometer-sized domains. Using morphological controls such as annealing and blending, we will template the self-organization of the block copolymer into an optimal structure that will force the charge recombination to occur within the phosphor portion of the molecule.

\section{Accomplishments}

A block copolymer synthesis procedure was developed in the past year to synthesize a symmetric diblock copolymer composed of a hole transport (HT) block and an electron transport (ET) block. The individual ET and HT polymers are chemically immiscible. The copolymer has been characterized as having a molecular weight of $30 \mathrm{~kg} / \mathrm{mol}$ for the ET block and $30 \mathrm{~kg} / \mathrm{mol}$ for the HT block with a polydispersity of 1.37 .

Two chemically immiscible materials will always want to segregate. Combining these two immiscible materials into a single molecule allows the development of a much more controlled microstructure in a single layer. Using the morphological control technique of vaporphase annealing, the above synthesized block copolymer self-assembled into a lamella structure. The domain spacing was determined to be $25 \mathrm{~nm}$. This self-assembled structure consisted of an ET block in one phase and a HT block in an adjacent phase. The self-assembled microstructure was independently confirmed by Small Angle X-Ray Scattering (a), Atomic Force Microscopy (b), and Transmission Electron Microscopy (c). Work is currently focused on synthesis of a triblock copolymer that incorporates a phosphor block, processing that provides morphological control, and PLED device fabrication and testing.

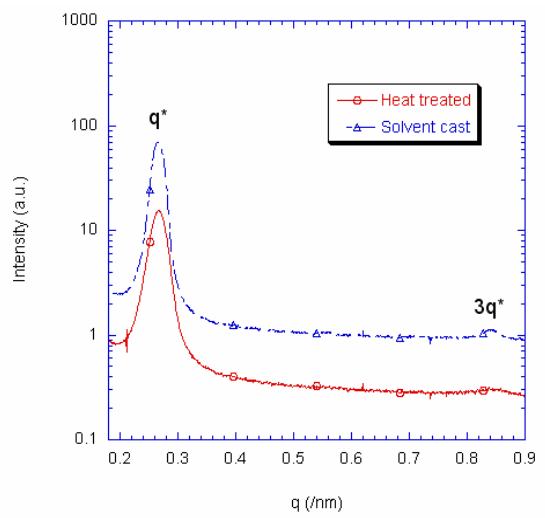

a

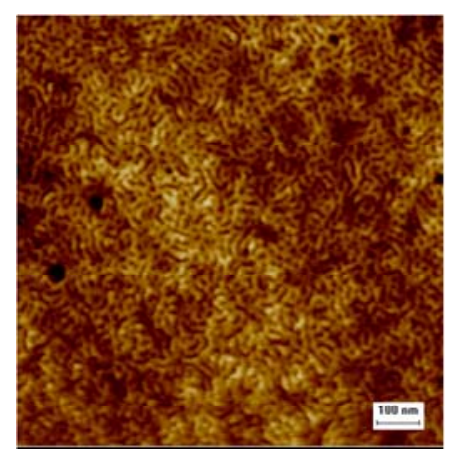

b

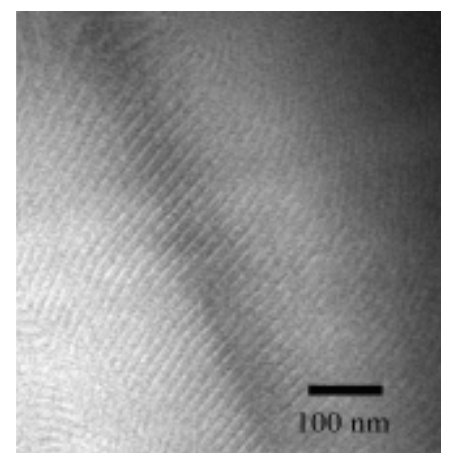

c 
Novel High-Temperature Membranes and Electrocatalyst Structures for Fuel Cells Principal Investigator(s): Nitash Balsara, Kyungoul Baek, Robert Kostecki

\section{Project Description}

Polymer-electrolyte (PE) fuel cells are in a dramatic period of rapid development, but there remain serious problems that need to be solved before they can achieve significant market penetration. These problems include significant efficiency losses due to the poor oxygen-electrode catalyst, prohibitive cost of current materials, and the need to find a suitable high-temperature membrane structure. The purpose of this project is to find new materials that address the above problems, and understand better the underlying fundamental processes. The two main goals of the project are: 1) develop new high-temperature proton-conducting membranes, and 2) study electrocatalysis and develop new catalyst structures to lower efficiency losses.

\section{Accomplishments}

The first goal was to resolve problems with current state-of-the-art PE membranes (PEMs) that are based on a specialized material. These membranes only operate at temperatures below 80 ${ }^{\circ} \mathrm{C}$. Above this temperature, water cannot be maintained within the membrane and proton conductivity is lost. We have overcome this limitation by constructing nanostructured PEMs using block copolymers. The nanoscale channels are designed to keep the PEM hydrated at temperatures above the normal boiling point of water by a combination of capillary condensation and chemical affinity. We are not aware of any other efforts in this field that have considered the use of capillary condensation as a mechanism for obtaining high proton conductivity. We have completed the synthesis of the initial set of block copolymers and determined their nanoscale morphology both in dry and hydrated states. Preliminary small-angle X-ray scattering (SAXS) experiments show that the nanostructure of our hydrated membranes does not change when the membranes are heated to temperatures as high as $120^{\circ} \mathrm{C}$. These results are being summarized in a publication.

To accomplish the second goal, our primary task was to develop a new diagnostic technique(s) to provide valuable information for designing nano-structured catalyst electrodes for the proton exchange polymer (PEM) fuel cells. We intended to correlate catalyst morphology with electroreactivity. To detect and characterize electrochemical processes at the nanometer scale resolution the dimensions of a nano-probe must be equal or smaller than surface morphology features at the electrode/electrolyte interface. We used commercial ultra-sharp silicon AFM tips with a custom made Pt coating. Another coating process was developed to insulate the nano-probes with thin non-conductive resin films, except for the tip's very end i.e, less than 20 nm diameter. This new probe was tested in situ on HOPG surface modified with randomly distributed and isolated Pt clusters of size ranging from a 30 to $500 \mathrm{~nm}$ in $0.1 \mathrm{M} \mathrm{HCl}$ aqueous solution saturated with $\mathrm{H}_{2}$. Potential distortions observed at the Pt nano-probe during a "pilot mode" scan 1-3 nm above HOPG/Pt surface upon intermittent polarization $0 \mathrm{~V}$ vs. NHE corresponded exactly to the location and morphology of the Pt particles. We also developed a new technique of microwaveassisted synthesis of Pt-graphite coatings for composite electrodes for fuel cells. This invention provides a novel method for simultaneous formation of electronically conductive graphitic carbon films decorated with uniformly distributed ultra-fine Pt particles on any type of substrate.

The above work will form the bases of our contribution to future multi-investigator DOE proposals in the area of fuel cells. 


\section{Nanostructured Cathodes for Efficient Organic LED}

Principal Investigators: Samuel S. Mao and Richard E. Russo

\section{Project Description}

The purpose of this project is to develop structured cathodes for achieving high efficiency organic light-emitting diode (OLED) devices. Since the remarkable discovery of electroluminescence from organic molecules and conjugated polymers, there has been an increasing interest in the development of OLEDs for lighting and display applications. However, a major limitation for OLED technology is inefficient injection of electrons from cathode to the organic electroluminescent layer.

A basic OLED structure consists of a thin layer of organic electroluminescent medium sandwiched between a cathode and an anode. With electrons injected from the cathode into the organic material's lowest unoccupied molecular orbital (LUMO) and holes from the anode into the highest occupied molecular orbital (HOMO), the device emits light upon the application of a voltage greater than a threshold bias. In principle, the cathode work function should be small enough to match the LUMO of the organic material, so that it is efficient to inject a steady supply of electron for light generation. While alkali metals have the lowest work function of all elemental metals, their highly reactive nature has been a major barrier for the realization of long lifetime, high efficiency OLED devices. It is therefore the objective of this research to look for alternative stable electrode materials to improve the efficiency of OLED devices.

\section{Accomplishments}

To overcome the barrier of inefficient electron injection in OLEDs, we developed a concept of utilizing stable nanoscale materials as the OLED cathode layer. In the past year, we have been focusing on developing OLED cathode layers using aligned carbon nanotubes.

Figure 1 shows aligned carbon nanotubes grown based on a nanostructured aluminum oxide template. Aluminum is easily turned into a template by anodization, through which an $\mathrm{Al}$ foil or film is transformed into self-organized nanoporous structure (nanoscale holes). Carbon nanotubes can grow inside each nanoscale hole, and by etching and removing the template layer, aligned nanotubes result. The size of the nanoscale holes of the template can be controlled by changing the time and the voltage applied during the anodization process. Measurements of current-voltage curves of fabricated OLED devices using a standard aluminum cathode and an aligned carbon nanotube cathode indicate, when aligned nanotubes are used as the cathode, the device appears to have an almost linear I-V relation instead of typical I-V curve with a high threshold, suggesting much-reduced resistivity for electron injection into OLED devices.

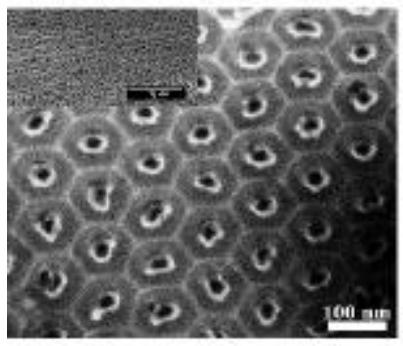

Figure 1. SEM image of an aligned carbon nanotube-based OLED cathode. The inset (top-left) in is an SEM image of an aluminum oxide nanostructured template. 


\section{Evaluating Aerosol Effects on Regional and Global Energy and Water Budgets Principal Investigator: Surabi Menon}

\section{Project Description}

A large component of the uncertainty in current climate projections/predictions are related to aerosol climate effects. Both aerosols (e.g. sulfates, nitrates, organic and black carbon) and greenhouse gases (GHGs) play a strong role in modulating the energy and water cycles. While climate effects from GHGs are relatively well quantified, aerosol climate effects through the direct, semi-direct, thermodynamical and indirect processes cannot be quantified without a large uncertainty due to complexities in evaluating these various processes. The work proposed through the LDRD project was focused on evaluating the role of absorbing aerosols on the energy and water cycles and advancing current understanding of aerosol-cloud interactions in relation to other climate forcings via a series of climate simulations.

We used the NASA Goddard Institute for Space Studies (GISS) coupled aerosol-climate model to assess the two issues listed above. Several climate simulations were carried out to understand: (1) effect of vertical distributions of absorbing particles on the energy and water budgets; (2) treatment of precipitation processes in the model and its response to changes in aerosol concentrations; (3) effects of aerosols on both convective and stratiform clouds; (4) relative role of aerosols versus other climate forcing processes in affecting climate trends for the last 50 years; (4) changes to future climate (2030) due to aerosol-cloud interactions.

\section{Accomplishments}

The effects of aerosols on climate during the Chinese summer monsoon were studied, based on simulations with the GISS climate model. We found greater changes in simulated temperature, radiation and precipitation for aerosols confined below $\sim 550 \mathrm{hPa}$, which suggest that climate effects of aerosols (especially absorbing aerosols) are stronger when they are located at higher atmospheric levels. In addition we also analyzed regional precipitation changes over China and India due to differing amounts of black carbon from fossil/bio-fuel sources. In both regions, precipitation changes appear to correspond to the amount of atmospheric heating, with stronger radiative fluxes (heating) in the atmospheric column corresponding to larger changes (positive) in precipitation though these may also depend on surface/meteorological conditions.

Regional changes in climate diagnostics were evaluated for aerosol effects on stratiform and convective clouds. Including aerosol-convective cloud effects lowers the indirect effect, and the level of precipitation initiation is shifter to higher atmospheric levels. This lower value of the indirect effect was due to decreased values of liquid water path due to aerosol suppression of precipitation in warm deep convective clouds that increases anvil formation. Transient climate simulations were also performed for 1960-2002 for two cases: (a) with all climate forcings (GHGs, solar irradiance, stratospheric water vapor, tropospheric and stratospheric ozone, volcanic aerosols, changes in land use, and snow/ice albedo and tropospheric aerosols including aerosol-cloud interactions); and (b) similar to (a) but for natural aerosols only. Overall, only with anthropogenic aerosols do we find a general reduction in land surface absorbed solar radiation for 1960-2002: a decreasing trend for 1960-1990 followed by an increasing trend for 1991-2002, consistent with observations. The implications of these results suggest that aerosols may no longer mask GHG effects over locations where aerosol emissions have reduced without a reduction in GHG levels. Future climate simulations for 2030 suggest that tropical regions may be subject to adverse air pollution and acid rain effects. 


\section{Analysis of High-Temperature Polymer-Electrolyte Fuel-Cell Phenomena Principal Investigator: John Newman}

\section{Project Description}

The purpose of this project is examine polymer-electrolyte fuel-cell (PEFC) behavior and possible operation at $120^{\circ} \mathrm{C}$. This temperature is above the maximum currently being used today $\left(\sim 80^{\circ} \mathrm{C}\right)$, and is thought to be key in the realization of the technology for transportation applications. While there is much research ongoing to find suitable individual materials and components that operate at $120^{\circ} \mathrm{C}$, there is a lack of understanding of how a PEFC operates at this higher temperature. For example, due to the much higher vapor pressure of water, the dilution effect of oxygen by the product water is expected to be more significant. Furthermore, though there is a gain in reaction kinetics, the open-circuit potential decreases with temperature, and hence there is a tradeoff between the useable energy that can be achieved by increasing the temperature. In addition, there are other, possibly unknown, tradeoffs such as the changes due to material properties like the conductivity of the membrane as a function of water content. Finally, the value of $120^{\circ} \mathrm{C}$ is chosen based on what is standard for current automobiles and not on optimization of a given PEFC system. It is the purpose of this proposal to examine these tradeoffs and optimize the system. Specifically, the main goals of the project are to understand, examine, and optimize the operation of a high-temperature PEFC through simulation.

In order to accomplish the goals, a numerical simulation will be developed. This involves a pseudo-two-dimensional macrohomogeneous model based on equations that describe the governing phenomena. This model will be validated against experimental results of cell polarization using experimentally measured parameters. Simulations will initially provide fundamental understanding and insight into the problems associated with high-temperature PEFC operation. Later, simulations will be used to optimize and examine the tradeoffs and effects of the various material properties such as conductivity, hydrophobicity, etc. These design criteria will provide realistic targets for experimental research and allow for high-temperature PEFCs to be developed.

\section{Accomplishments}

The most significant accomplishment is the development of mathematical code to describe PEFC operation at $120^{\circ} \mathrm{C}$. The 1-D and pseudo 2-D code has been written, tested, and debugged. The code contains the underlying physical phenomena including two-phase flow of liquid (water will form inside very hydrophilic pores) and vapor, detailed reaction-layer kinetics with microstructural information, and a robust description of water and proton movement in the membrane. The 2-D framework allows for examining different flow arrangements such as counterflow and coflow of the reactant gases.

The model has undergone initial validation by fitting experimental polarization-curve data. This is done with a minimum number of fitting parameters, with the majority of the values coming from literature sources. With the second year of obtained LDRD funding, we plan to finish validation of the model and quantitatively examine the tradeoffs of high-temperature operation, as well as determine the limiting phenomena and optimize material properties. 


\section{Long-Term Global Energy Demand and Carbon Emissions Scenarios}

Jayant Sathaye, Mark D. Levine, Lynn Price, Stephane de la Rue du Can, Michael

McNeil, Shyam Menon, Virginie Letschert, Phil Price, Jonathan Sinton, and Nan Zhou

\section{Project Description:}

The Intergovernmental Panel on Climate Change (IPCC) has developed a set of global scenarios that report on the anthropogenic greenhouse gas emissions from all sources up to the year 2100 by ten geographic regions. The scenarios were developed using a set of models that lacked a detailed representation of demand-side drivers and technologies. These scenarios provide the overall conceptual framework in which emissions of greenhouse gases and their impacts are analyzed.

The purpose of this project is to develop scenarios of energy demand by end-use for ten global regions consistent with one or more IPCC scenarios. Surprisingly, the global energy demand scenarios developed by IPCC, other intergovernmental organizations (e.g., UN, International Energy Agency (IEA)), international oil companies, and national governments are lacking in end-use detail. Most of the scenarios provide no more detail than total energy use by buildings, industry, and transport (by region, energy type, and year). Little if any of the underlying drivers (energy service demand, technology type, usage, saturation, efficiency) are included in the scenarios. As a result, it is virtually impossible to understand or interpret the scenarios in terms of current or future energy demand. The LDRD project will for the first time provide such information to the community that analyzes climate change.

\section{Accomplishments:}

We have disaggregated two scenarios by sector (buildings, industry, transportation, and agriculture) for 10 world regions. This information was provided to the IPCC WGIII chapter authors for use in the First Order Draft of Assessment Report 4. Additional work to further disaggregate the sectors into residential and commercial buildings as well as major industrial sectors has begun.

We have designed a Global Energy Demand model and begun collection of publicly-available data as well as data provided by collaborating research entities worldwide. We disaggregate each end-use sector at three different levels of detail, starting at the macro level and working to the third level that contains end-use technologies. Using this approach allows us to use level 3 - end-use technology -- data for "marker" countries in a region to understand the possible energy consumption trends in the remaining countries in that region.

We have completed Level 1 by integrating historical energy consumption, GDP, and population data from the IEA with the IPCC scenarios for all countries. We are in the process of designing and gathering data for Level 2, which is the level that will be used to understand driving forces for the sectors by region. We have developed the structure for Level 3 for the buildings sector and are expanding it to the other sectors. We have populated the model with data for China and have begun to collect data for the U.S., Japan, and India. We are working with institutions in other countries to assist us with this data-collection effort, including the Asia Pacific Energy Research Centre (APERC) based in Japan, which will work with LBNL in procuring demand-side data and information from their 22 member countries. 


\section{Genomics Division}

LB04026

\section{Making the Most of Sequencing: Improved Assembly and Comparative Annotation \\ Principal Investigator: Eugene Myers}

\section{Project Description}

Our goal is to efficiently sequence and annotate genomes using a comparative approach. We are building a whole genome assembler that will deliver $99-99.5 \%$ of a genome as opposed to the 97\% typically returned by today's software. A highly sensitive, yet efficient whole genome aligner is being built that will find the most-likely evolutionary relationships between genome segments and will further correctly align sequences at the base-pair level. Finally, a symmetric comparative gene-finder that simultaneously finds the best annotation of all orthologs in the input genomes is underway. A similar RNA-gene finder and cis-regulatory element finder are also potential objectives. Each component feeds the next: more efficient assembly permits more sequences, each more complete as input to the comparative step, and better whole genome alignments are critical to more robust comparative gene finding.

A better assembler requires better repeat resolution and we will pursue a number of algorithmic strategies that attempt to exploit micro-heterogenieties between repeats and the integrity of paired reads. But before separating repeats, one must first be aware of them and their structure. We develop a "string graph" concept where repeats are collapsed but clearly visible. Our whole genome comparator is based on (1) novel methods for rapidly finding local alignments, (2) a maximum-likelihood based approach to genome rearrangements, and (3) sophisticated gap-scoring schemes to ensure that the base-level details of an alignment are correct. Finally, the best possible use of comparative data is to build the best possible 1dimensional gene finders (i.e. HMMs) and then constrain them to predict the same orthologous protein in all $\mathrm{k}$ species simultaneously.

\section{Accomplishments}

This year work on the assembler continued with Michael Brudno and Stuart Davidson supported on the LDRD. A novel algorithm for finding local overlaps very efficiently (50 times faster than Phil Green's swat for example) has permitted us to build a "scruber" that effectively removes all vector sequence and chimeric constructrs prior to assembly proper. This has a critical effect on our string graph approach to assembly. We further developed a complex network flow approach to assigning repeat labels to the string graph in order to find feasible

tours. Preliminary progress was made on using mate pairs to further elucidate the correct tour through the graph. Algorithms to convert the labeld string graph back to a reconstructed sequence and consensus multi-alignment were developed. In effect we reached the state of an end to end assembler and are now analyzing its performance on medium scale genomes in order to understand remaining weaknesses in the approach.

With Bob Edgar we developed methods to identify repetitive segments of a genome as a prelude to whole genome alignment. The method is highly accurate and we have discovered 100s of new elements in Arabadopsis and the twelve flies that have been recently sequenced. With Ariel Schwartz we realized that existing concepts of alignment accuracy were flawed and favored over-alignment of the sequence. We developed a new measure and an algorithm for finding alignments that optimize accuracy as part of our work on a comparative gene finder. Ariel also continues to make progress on our overall approach for this work and intends to complete it as his $\mathrm{Ph} . \mathrm{D}$. thesis. 
Transgenic Mouse Production and Identification of New Genes in Transgenic Mice Principal Investigator: Eddy Rubin

Project Description:

Transgenic animals have been genetically modified to contain extra copies of normal or mutated genes and/or DNA sequences. Knockout animals produced by gene targeting have deletions in specific chromosomal regions. These animals are invaluable research tools for gene discovery, study of gene regulation, and creation of models of human diseases in mice. Considerable significance now appears to be in the role of the noncoding sequences in the genome, the functions of which are only beginning to be addressed. By coupling our expertise in comparative genomics and high throughput mouse transgenesis, we have succeeded in using these mouse models to verify the enhancer activity of a set of highly conserved noncoding elements. Putative enhancers are first identified using stringent computational and comparative genomic filters and then tested for activity using an in vivo transgenic mouse reporter assay.

For this project, a new approach utilizing the Lentivirus will be used as a gene delivery vehicle to generate transgenic mice for the analysis of gene enhancer elements. This injected virus also will carry the LacZ Reporter gene for confirmation of gene presence. The lentiviral vector we use was developed in the laboratories of Inder Verma, Didier Trono, and Fred Gage (Salk Institute) and Luigi Naldini (Cell Genesys). It is one of the newest generation vector based on humnan immunodeficiency virus (HIV-1.

Accomplishments:

Strict selection of human elements, which are maximally conserved with orthologous segments in rodents, has identified 481 ultra-conserved elements longer than 200 bp sequences with 100\% sequence identify (Bejerano et al, Science 304(5675): 1321$5)$. During the past year and this project, we have tested 34 human-rodent ultra-conserved sequences for enhancer activity using mouse transient transgenesis assay and found that 19 elements (56\%) strongly modulate tissue-specific expression of B-galactosidase reporter construct at embryonic day 11.5. This has led to the identification of enhancers for forebrain, midbrain, hindbrain, eye, dorsal root ganglion, limbs, and neural creast. The results of this study are available in a public database that we have established to visualize the expression patterns and the localization of genome-wide human-fugu and human-rodent conserved elements (url: http://enhancer.lbl.gov/). Taken together, these studies supp0ort the utility of comparative genomics and transient transgenesis to identify and characterize gene regulatory elements scattered throughout the human genome and provide insights into regulation of gene expression. 


\title{
Life Sciences Division
}

LB04010

\author{
Molecular Electron Microscope Tomograph \\ Principle Investigator: Manfred Auer
}

\section{Project Description}

The purpose of this project is to employ electron microscopy tomography in combination with novel genetic tagging and biochemical labeling methods to study the three-dimensional localization and molecular three-dimensional structure of macromolecular machines in their native cellular environment. The goal is to identify the constituting protein components and to determine the architectural building principle of cellular machines. Our focus lies on macromolecular machines that transduce mechanical signals across biological membranes, e.g. in inner ear hair cells underlying our sense of hearing and balance as well as focal adhesion complexes in the context of breast cancer metastasis.

Inspired by the DOE-GTL initiative we have begun to apply these powerful imaging and labeling techniques to bacterial colonies of Myxococcus xanthus with an initial focus on the unexplored interbacterial extracellular space, as well as to Desulfovibrio vulgaris biofilms with the aim to localize at high resolution up to 400 tagged proteins throughout representative portions of the biofilm under normal as well as various stress conditions.

Since biofilms are the preferred life style of bacteria with a vastly altered protein expression pattern compared to bacterial broth culture conditions, we believe that our efforts to study macromolecular machines in biofilms is highly significant to the mission of the Department of Energy and its GTL initiative. Only when the precise localization pattern of the proteins within the cells under various conditions is known, will we be able to mathematically predict bacterial behavior under stress conditions that are usually found at DOE bioremediation sites.

\section{Accomplishments}

We have examined the structure of the mechanoelectric transduction and adaptation machinery of the inner ear hair cells, which from an engineering point of view is unsurpassed in its elegant and efficient design. We were able to visualize the complexity of this molecular machine and found an auxiliary link that has been overlooked to date. Based on our tomographic data, we propose fibronection as the candidate protein for the long-sought-after tip link. We have accumulated a body of evidence that supports our hypothesis and are currently trying to prove this hypothesis beyond doubt using quantum dot labeling of biotinylated fibronectin fragments, which we have shown can interfere with the recovery of tip links in zebrafish after low-calcium treatment.

With respect novel tagged-based labeling approaches, we have succeeded in carrying out a pilot study in a cell culture system and are currently extending this novel labeling approach to transgenic zebrafish as well as bacterial biofilms.

Our bacterial biofilm study has yielded remarkable results, and has led us to the conclusion that bacteria may use extracellular vesicle trafficking for cell-to-cell protein transfer. We are currently biochemically analyzing the content of purified vesicles and found $\sim 15$ protein, lipids as well as nucleic acids. We anticipate that this result will cause a paradigm shift and alter the way we think about bacteria and microbial communities.

The LDRD funds have been instrumental to successfully compete for the GTL-grant on Desulfovibrio. 


\section{Response of Chromatin Structure in Mammary Epithelial Cells to Microenvironmental Cues \\ Principal Investigators: Felicia Betancourt and Mina Bissell}

\section{Project Description}

Normal mammary epithelial cells respond to extracellular messengers such as lactogenic hormones by expressing beta-casein and other milk proteins. However, for reasons that are not well-understood, malignant cells from this tissue have lost the ability to respond to these cues. We ultimately seek to understand how protein-DNA interactions at the promoters of genes encoding milk proteins change when signals for milk production are presented to both normal and malignant mammary epithelial cells. Our immediate goal is to examine the structure of chromatin (broadly speaking, any and all proteins assembled on target DNA) reconstituted on the beta-casein promoter from mouse mammary epithelial cells grown under conditions that do or do not lead to milk production. The detection and analysis of protein-DNA complexes will be accomplished by 1) capture of proteins binding to biotinylated beta-casein promoter sequences immobilized on streptavidin beads; and 2) visualization of complexes by cryoelectron microscopy.

\section{Accomplishments}

During the period of this LDRD, we were able to

- show that by choosing the right ionic strength, transcription factors known to bind at the beta-casein promoter could easily be extracted from the nuclei of EpH4 cells, a non-transformed mouse mammary epithelial cell line;

- show that at least one of these transcription factors binds tightly and stably at the promoter; and

- show that naked DNA the same size as our promoter fragments can be identified in cryogenically prepared specimens in the electron microscope (see figure). This last point is particularly good news, since complexes of protein-bound DNA should be even easier to distinguish than naked DNA. Our immediate goal is to identify protein-DNA complexes in the microscope, and then use image processing software to get information about the $3 \mathrm{D}$ structure of the complex or complexes.

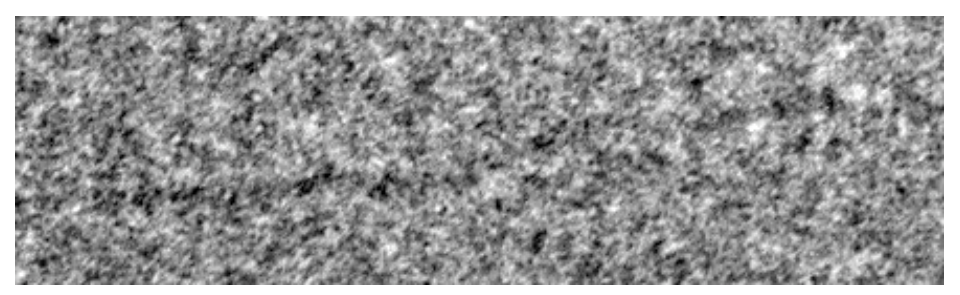

Figure: a molecule of DNA 331bp long imbedded in vitreous ice and imaged at a magnification of 50,000x. 


\title{
A High Throughput Strategy to Identify Protein Complexes
}

\author{
P.I.s Mark D. Biggin, Jian Jin, Ursula Schulze-Gahmen
}

\section{Project Description}

A major focus of current DOE research directions is to identify and characterize the dynamic changes in protein complexes on a genome wide scale. We propose a novel strategy to purify and identify stable protein complexes with much higher throughput than current methods.

The most common strategy to isolate protein complexes from crude cell extracts uses protein specific affinity tags. Each tagged complex must be purified one-at-a-time from a distinct genetically engineered strain. Then, polypeptides in the complex are identified by mass spectrometry. Our novel method, termed the "tag-less" strategy, uses a multidimensional, highly parallel chromatography platform to divide a crude bacterial extract into $10,000-20,000$ fractions, then use LC MALDI mass spectrometry to identify the polypeptide constituents in each fraction from a tryptic digest of the whole fraction. This strategy should permit rapid identification of most stable water soluble complexes in an organism from one preparation of cells. During the course of this pilot project, we have generated and analyzing several hundred fractions, including some that contain known protein complexes as positive controls. To validate our tag-less strategy, we are comparing the known and putative complexes purified in this way to the same complexes isolated using the standard tagged strategy for affinity purification.

\section{Accomplishments}

To pilot the tagless strategy, we first identified by western blot and purified bacterial RNA polymerase. From a $6 \mathrm{~L}$ culture we obtained $1 \mathrm{n}$ mol of pure protein. We showed that we can efficiently detect all polypeptides in this complex using only $1 / 10,000$ of this preparation of protein using both LC ESI Qtof and LC MALDI tof/tof mass spectrometry methods. RNA polymerase is a relatively abundant protein, present at around 600 molecules per cell. But the fact that we can easily detect this implies that with improved sample concentration we should be able to detect the lowest abundance complexes present at one molecule per cell.

To test if the blind screening approach at the heart of the tagless strategy is feasible, we have first performed SDS PAGE on a series of fractions that in effect search a small part of our proposed multi dimensional parallel chromatography scheme. Every other fraction from the mono $\mathrm{Q}$ fractions used to purify RNA polymerase were each subjected to fractionation by hydrophobic interaction chromatography (HIC). Manual inspection of these fractions reveal five sets of co-migrating polypeptides that were then identified as know protein complexes.

We have also established the affinity tag method to isolate two E. coli complexes: acyl carrier protein (ACP) and RNA polymerase. We correctly identified the known subunits of RNA polymerase and detect a set of polypeptides associated with ACP.

An important technical hurdle we must overcome is to show that MALDI mass spectrometry can provide sufficiently quantitative data that the relative enrichment of polypeptides can be judged between adjacent fractions. We have demonstrated this is feasible by adapting iTRAQ technology to our method.

Finally, in the last few months of the LDRD project, we have initiated studies on Desulfovibrio vulgaris that will complement a large Genomics: GTL project, whose funding commenced directly after the LDRD funding ended. We have established an efficient protocol to extract soluble proteins and have identified several complexes using the tagless strategy. 


\author{
Modeling Human Disease Genes In Drosophila Melanogaster \\ Principal Investigator: Susan Celniker
}

\title{
Project Description
}

Many of the genes that are deregulated by genomic aberrations in human cancers are conserved between Drosophila and humans. Our goal is to develop Drosophila-based analyses of human epithelial cancer gene functions by (a) designing strategies to efficiently generate strains in which candidate genes are inactivated or ectopically over-expressed (b) identifying functionally important genes in embryos using automated techniques to assess growth, proliferation, apoptosis and pattern formation in gain-of-function and loss-of-function mutants; and (c) locating candidate genes within specific pathways (epistatis analysis) for which morphological readouts exist using semiautomated approaches to assess adult fly reporter phenotypes. Specifically we plan to apply high throughput technologies and the power of Drosophila genetics to dissect cancer gene function and identify new components of cancer pathways.

\section{Accomplishments}

We focused on genes in regions that undergo high levels of amplication in patients with short survival ovarian cancer and with breast cancer near the ErbB2 amplicon. We have identified cognates for eleven genes. RNAi and UAS transgene constructs to interrogate loss and gain of gene function, respectively are underway. We found that Breast carcinoma amplified sequence 4 (BCAS-4) is similar to the human gene cappuccino, a member of the BLOC-1 complex required for normal vesicle trafficking to lysosome. The process of endocytosis has recently been linked to the control of cell proliferation and defects in endocystosis to a role in neoplasia.

To investigate the role of endocytosis in cancers, we have developed eleven RNAi constructs driven with a UAS promoter to generate conditional knockdown of gene functions. We targeted the Drosophila cognates of genes in the endocytosis pathway including the Rab proteins, RB4 and Syntactin 13, the Rab effectors, rabaptin and rabenosyn, co-factors Sly1 and Vps45 and multivesicular body (MVB) escort II protein, Vps25 and escort III proteins, Vps2, Vps20, Vps 24 and Vps32. For eight of these genes we have generated between 10 and 46 different RNAi insertion lines. Two more have been injected and we are awaiting the transformation results. To generate lethal excisions or complete loss-of-function alleles we have identified P-element insertions near five of the genes and have generated excisions that are lethal over deficiencies. Phenotypic characterization of these mutants is being performed in collaboration with David Bilder's laboratory (UCB), experts in studying neoplastic tumors in Drosophila. Our most significant results to date show that ectopic expression of RNAi constructs for Vps25, Vps32 and Sly1 result in larval lethality. Conditional knockdown of gene function of Vps25 in the imaginal wing disc results in a crumpled wing phenotype.

RNAi is extremely powerful but unfortunately limited by position effects on expression. To minimize position effects we have developed a new Drosophila RNAi vector for transgenics that contains the site specific recombination element, attB, allowing targeted integration. We are screening over 40 attP lines to find the best genomic environment for our studies.

Finally, in related work, in collaboration with Jian Jin's LBNL engineering group, we are building two automated systems. One for imaging single adult flies using a CCD camera and pattern recognition software. This system will sort flies based on eye color and size, wing shape and sex. The other for microinjection of embryos. We expect to have working systems by June of 2006 . 


\section{Unmasking the Human Genome Alternative Splicing Program Principal Investigator: John Conboy}

\section{Project Description}

Alternative pre-mRNA splicing is a major mechanism for regulation of gene expression in the human genome, allowing a relatively small number of genes to encode a highly complex proteome. However, previous microarray technologies for quantitating mRNA output from each gene fail to distinguish qualitative differences in mRNA, generated by alternative splicing, which may completely alter gene function. Relatively little is known about how the alternative splicing program is coordinated during normal differentiation and development, or how aberrations in the process contribute to human disease including cancer. The major goals of this project are (1) to use novel exon microarrays and bioinformatics tools to illuminate genome-wide, tissue-specific patterns of alternative splicing, and (2) to identify evolutionarily conserved regulatory sequences that interact with the splicing machinery to orchestrate the human genome alternative splicing program.

\section{Accomplishments}

During the first phase of our LDRD project, we finished a pilot study of a small group of brain-enriched exons that had been identified via low throughput pre-array technology. As our major novel finding, we identified computationally a unique 6-nucleotide regulatory motif that we hypothesize represents part of a fundamental "switch" mechanism for activation of tissuespecific alternative exon splicing, and is therefore critical for functional specialization of differentiated cell types. This element is specifically associated with tissue-specific alternative exons, but not control exons, and is evolutionarily conserved in mammalian, avian, and piscine genomes. These studies were performed in collaboration with Inna Dubchak of the Laboratory's Genomics Division, and were recently published in Nucleic Acids Research.

The next phase of the project focused on the first high throughput, genome-wide data generated by the new Affymetrix exon microarray. As proof of principle that the array performs as expected, the data revealed a novel set of previously unappreciated muscle-enriched exons. Many of these map to genes encoding cytoskeletal proteins, suggesting the intriguing hypothesis that a functionally coherent group of genes is coordinately regulated by a carefully orchestrated program of alternative pre-mRNA splicing. Biochemical and computational studies now focus on a flanking intron sequence motif that we hypothesize to be a critical part of the machinery for alternative splicing switches during development. A manuscript describing these studies in collaboration with Inna Dubchak, Debopriya Das, and Affymetrix scientists John Blume, Tyson Clark, and Tony Schweitzer, is in preparation.

Finally, in collaboration with Life Sciences Division Director Joe Gray, and the Affymetrix team, we are in the early phase of analyzing aberrant alternative splicing patterns in a large series of human breast cancer cell lines. The long range goal of this analysis is to determine the incidence and biological importance of aberrant pre-mRNA splicing in tumor biology.

In conclusion, the results of this LDRD support the hypothesis that highly coordinated programs of alternative pre-mRNA splicing can be elucidated through the use of exon microarrays. This strategy should provide fundamentally new insights into biological systems important to several existing LSD programs, and facilitate new mechanistic studies of the splicing regulatory networks that control tissue-specific splicing. These new insights may ultimately suggest therapeutic approaches to aberrant splicing in human cancer. 
Improved Phase Contrast for Cryo-EM of Biological Machines and Subcellular Structure Principal Investigators: R. M. Glaeser, Jian Jin, Roar Kilaas, Kenneth Downing, Eva Nogales, Manfred Auer

Project Description

The goal of this project is to use a microfabricated electrostatic device to selectively apply a 90 degree phase shift to the central (unscattered) beam in the back focal plane of the objective lens of an electron microscope. This phase-shift device will provide "ideal" phase contrast for in-focus electron microscopy of biological specimens. This concept would have been unthinkable without the development of micrometer-scale threedimensional fabrication techniques, and without the even more recent, commercial development of electron-optical aberration correctors. Significant engineering challenges must still be addressed, however, to prevent electrostatic charging of the phase-plate device and to keep it centered on the unscattered electron beam.

The long-term opportunity, if this project is successful, will be to achieve many of the ambitions articulated in the DOE statement of goals for its proposed GTL Facility within the areas of macromolecular and cellular imaging. The short-term need is to acquire sufficient preliminary results on which to base strong proposals for long-term grant funding in the areas of single-particle cryo-EM and EM tomography.

Accomplishments

A novel, 2-electrode Einzel lens was investigated as a possible phase-contrast device. This design was chosen because of the high likelihood that it could be fabricated much more easily than the more conventional, 3-electrode Einzel lens. Electrostatic calculations were used to show that a 5 micrometer long central tube, surrounded by a grounded "guard" electrode, would produce only a negligible fringing field in the region of the scattered electrons, while providing the desired phase shift for the unscattered electrons. Heat-transfer calculations were then used to show that the heat-load on the specimen will be negligible when the central electrode is heated to $200 \mathrm{C}$, which is high enough to prevent beam-induced contamination and subsequent beam-induced charging of the device. A patent covering the use of this design to provide phase contrast in electron microscopy has been submitted.

Preliminary microfabrication tests were successful in making a prototype with smallest feature sizes of 2 micrometers. The quality of fabrication achieved with the prototype was so good that we are confident that the feature sizes can easily be reduced to 1 micrometer, which is our initial design goal.

A detailed draft-design was developed for maintaining the position of the device with the needed, sub-micrometer accuracy, and commercially available components were identified that could be used when this design is implemented. All necessary theoretical investigation and preliminary design work has thus been completed.

A second year of LDRD funding has been awarded for this project. These funds will be used to build a working prototype with which we can demonstrate the validity of the design. 


\section{Functional Interpretation of Cancer Genomes}

Principal Investigator: Joe W. Gray

\section{Project Description}

The purpose of this project is to facilitate study of how genes and the proteins they encode interact in human normal and diseased cells. Generating this body of knowledge and using it to improve disease management is one of the next major challenges in biology and medicine. This is daunting because of the complexity of the interacting systems - ultimately, thousands of genes and gene products may be involved in important cellular processes such as growth, death, motility, angiogenesis and proteolysis. Advanced analytical and computational technologies developed around existing strengths in engineering, computer science, structural biology and biology at Berkeley Labs are being developed to address these problems. Our initial focus has been on cancer. Specifically, we have developed a cancer analysis "system" comprised of dozens of cancer cell lines that mimic important aspects of the genetics and biology of primary tumors and that capture the person-to-person variability found in primary tumors.

\section{Accomplishments}

We have expanded our information base on the genomic, epigenomic, gene expression and proteomic characteristics of a collection of breast and ovarian cancer cell lines that mirror many of the molecular features found in primary tumors. The high concordance suggests that the cell lines do reflect much of the biology found in primary tumors and so can be used to study these aspects of cancer biology and how they vary between individual tumors.

We have completed the design and construction of a semi-automated system for high throughput analysis of molecular (DNA, RNA and protein) and cellular responses (motility, cell death, proliferation rate, pathway activity) to therapy.

In this system, stock cell lines grown and maintained in a central cell culture facility are transferred from flasks and seeded into smaller volume multi-well cell culture plates, transported into and out of a multi-plate incubator as needed, fed and inspected and treated with agents that modulate expression of selected genes or biological pathways in order to study the resulting molecular and biological responses. Cell properties are analyzed using an automated microscope. Computational approaches have been developed to manage and analyze the resulting data.

This LDRD research supported an application in response to the NCI Integrative Cancer Biology Program which resulted in the award of $\$ 13,000,000$ over a 5 year period to develop and validate a computational model of cancer-related signaling networks. In addition it has led to the establishment of public-private sector research projects with GlaxoSmith Kline and Lilly and is supporting aspects of a Program Project in Ovarian Cancer now being developed at LBNL and the Bay Area Breast Cancer SPORE at UCSF. 
Computational Analysis of Structural Impacts of Exocyclic DNA Adducts on Specificity and Efficiency of Human Repair Enzymes

Principal Investigators: Bo Hang and Anton B. Guliaev

Project Description

Exocyclic adducts represent a unique group of base derivatives formed by the reaction of DNA with exogenous carcinogens or by endogenous metabolic processes. Several DNA repair enzymes in the base excision repair pathway have been found to repair these adducts. Studies by us and others have shown that their repair specificity and efficiency toward these adducts significantly vary with differences in exocyclic ring structures such as size, number, and substitution.

The purpose of this proposal is to use advanced computational methods, namely molecular dynamics simulations (MD) and quantum mechanical calculations, to systematically study the adduct-induced DNA conformational changes as well as interactions between an exocyclic adduct and the active site in DNA/enzyme complexes. By analyzing both substrate and nonsubstrate adducts, we wish to identify specific structural features important for recognition and catalytic activity of two human enzymes, alkylpurine-DNA glycosylase (APNG) and the major apurinic/apyrimidinic endonuclease (APE1). Such analyses and developed theories will also be verified, when necessary, by site-directed mutagenesis. This should greatly enhance our understanding of how selective repair takes place. Studies using adducts with unknown repairability may help to test whether this type of approaches is able to predict repair specificity based on structure and chemistry.

\section{Accomplishments}

We have applied MD to study structural aspects of recognition and excision by hAPNG toward a glycidaldehyde-derived adduct 7-(hydroxymethyl)-1, $\mathrm{N}^{6}$-ethenoadenine (7-hm-EA). Data produced allow us to identify specific conformational features from the adduct-containing DNA duplex as well as from the adduct-containing DNA complexed with APNG, as compared with the model substrate $\varepsilon A$. The hydroxyl group on the exocyclic ring of 7-hm- $\varepsilon A$ was shown to be involved in hydrogen bond interactions with the opposite base in DNA and the side chains of the APNG active site residues Asn 169 and Cys 178. Mutational studies showed that the replacement of Asn 169 by Ala or Ser significantly increases the excision efficiency of the APNG mutants toward 7-hm- $\varepsilon$ A, confirming the role of Asn 169 in substrate selectivity.

To gain structural insight into the mechanism of recognition and processing by hAPE1 of the benzene-derived adduct, $\mathrm{pBQ}-\mathrm{C}$, we previously performed $\mathrm{MD}$ on $\mathrm{pBQ}-\mathrm{C} / \mathrm{APE} 1$ complex. The position of $\mathrm{pBQ}-\mathrm{C}$ at the enzyme active site is stabilized by two unique hydrogen bonds formed between the adduct and the side chain of Asp 189 or the main chain amine of Ala 175. To verify this proposed role of Asp 189, two mutant proteins were generated under this LDRD: D189L and D189E. Biochemical experiments with these mutants indicate that D189 is an essential residue for the pBQ-C activity, agreeing with our molecular modeling finding.

These two parallel studies demonstrate that by using computational studies, in conjunction with biochemical data, we are able to identify important structural features and specific DNAprotein interactions, at the molecular level, that are essential for understanding the mechanism of actions of human repair enzymes, such as APNG and APE1. 


\section{Neuroimaging with Advanced Molecular Probes \\ Principal Investigator: William Jagust}

\section{Project Description}

The focus of this project is to develop methods for the non-invasive quantitation of the dopamine system and methods for quantitating the development of brain amyloid. Initially, our plans were to apply several ligands for the study of dopamine. We have revised our project to instead focus upon relationships between dopamine measurements and brain activation using functional MRI (fMRI) techniques. We have thus studied a group of older individuals in an effort at investigating relationships between declining dopamine function (measured with the tracer fluorometatyrosine, or FMT), brain physiology, and aging in order to probe mechanisms of compensation in the aging brain.

With regard to the amyloid studies, we have successfully deployed the tracer "Pittsburgh compound B" or PIB in order to investigate binding properties and quantitative methods for detecting amyloid in the brains of living humans. Amyloid is the protein deposited in the plaque in Alzheimer's disease. The goal of this project was to investigate the detection of amyloid in normal older people and those with dementia.

\section{Accomplishments}

In the past year we have completed studies on 15 additional subjects, all of whom are over the age of 50 and who have had PET studies using the tracer FMT in combination with fMRI measures of brain activation. We have perfected techniques for acquiring and quantitating these data. We have decided to use a graphical method for defining net tracer uptake (ie a Patlak plot), which obviates the need for arterial sampling. Because dopamine is particularly associated with working memory function, we tested participants using a listening span test that measures working memory, and also used a working memory paradigm during the fMRI study. Several key findings have emerged. First, we have found that individuals with lower FMT uptake in the caudate nucleus, indicative of lower dopamine production, have poorer working memory ability. Furthermore, we find that individuals with poorer working memory show less brain activation during the working memory task. Interestingly, for a given amount of FMT uptake (or dopamine synthesis) individuals who perform better on working memory tasks show greater brain activation. This suggests that individuals with greater dopaminergic resources recruit more neural resources to perform the cognitive task better than those with less dopamine.

As of the end of the 2005 calendar year we will have studied a total of 23 subjects with the amyloid imaging agent PIB. We are currently still in the process of analyzing data from this study. We have completed analysis of 11 subjects' data. We have found that, using a quantitative method to define tracer retention (the Logan plot to caculate distribution volume ratios or DVR), the technique shows $100 \%$ sensitivity and $71 \%$ specificity for the diagnosis of Alzheimer's disease compared to Frontotemporal lobar degeneration (FTLD), a non-amyloid dementia. We believe that the cases of FTLD which showed tracer uptake were, in fact, misdiagnosed. Thus, we suspect that this technique is going to be a highly effective method for determining the cause of dementia during life when the differentiation hinges on separating amyloid depositing from non-amyloid depositing dementias. 


\section{Imaging Signaling Networks In Normal And Malignant Tissue \\ Principal investigators: G. Steven Martin and Mina Bissell}

\section{Project description}

A typical mammalian cell contains several thousand signaling proteins that are organized into a complex signaling network. These signaling proteins function at a wide variety of intracellular locations and many translocate to other locations upon activation or inactivation. The goal of this project is to develop and use fluorescence imaging methods to follow the activation, translocation and membrane interactions of key signaling proteins in normal and malignant cells.

This project represents a three-way collaboration between the groups of Martin and Bissell (LBNL) and the group of Prof. Yoav Henis at the University of Tel Aviv. We have been focusing on the non-receptor tyrosine kinase Src, because this molecule is a central mediator of growth factor and integrin signaling. Src interacts with the internal plasma membrane leaflet through two N-terminal motifs - a myristoylation domain and a cluster of basic amino acid residues. Mutation of the myristoylation site blocks cell transformation by activated Src, indicating that membrane association plays a critical role in Src signaling. These interactions are likely to be significantly modulated by interactions with other proteins via the SH2 and SH3 domains of Src, as well as by its kinase activity. The goal of our research in the past year has been to use fluorescence recovery after photobleaching (FRAP) to investigate the role of the various domains of Src in defining its membrane association and the relationship between this association and Src activity.

\section{Accomplishments}

We have made use of a novel biophysical approach based on a laser beam-size test to distinguish between fluorescence recovery by lateral diffusion and fluorescence recovery by exchange with the cytosol. During the past year, we have generated a series of GFP-tagged c-Src mutants for these studies. Our experimental analysis indicates that we can measure exchange of Src between membrane-bound and cytosolic states as well as the lateral diffusion of wild-type and mutant forms of Src. The results substantiate our hypothesis that both Src kinase activity and its $\mathrm{SH} 2$ and $\mathrm{SH} 3$ domains modulate its membrane interactions.

The results of the FRAP experiments indicate that wild-type Src-GFP, which exists primarily in a closed inactive conformation, has a significant degree of association with the plasma membrane, but does undergo exchange with the cytosol. Its diffusion coefficient is close to that of a fluorescent lipid probe, indicating that it is not significantly associated with membrane proteins. Src can be rendered constitutively active by introduction of the Y527F mutation. Constitutively active Src527F-GFP shows a considerably reduced fluorescence recovery rate as compared to Src(wt)-GFP, as well as a significant decrease in exchange with the cytosol. Inactivation of Src catalytic activity by introduction of an inactivating mutation to the openconformation Src (Src527F/K295M-GFP) abolished the reduction in the FRAP rate due to the Y527F mutation. A similar effect was observed with a double mutant in which the binding capacity of the SH2 domain is impaired (Src527F/R175A-GFP). These results suggest that activated $\mathrm{Src}$ is associated via its $\mathrm{SH} 2$ domain with membrane proteins that it has phosphorylated. This hypothesis has been confirmed both by in vitro affinity precipitation methods and by in vivo competition experiments. Further studies are planned using bimolecular fluorescence complementation to examine the association of Src with specific substrates. 


\section{Materials Sciences Division}

LB05004

Ultrafast Measurements of Charge-ordering/disordering in Manganites: Development of a Femtosecond Electron Diffraction Probe

Principal Investigators: Andrea Cavalleri, Robert W. Schoenlein, and Nigel D. Browning

\section{Project Description:}

The goal of this project is the design, development, and construction of an ultrafast electron-diffraction apparatus to study geometric structural rearrangements of ordered electron lattices. The proposed first scientific application will be the study of charge ordering/disordering dynamics during the metal-insulator transition in a perovskite manganite. These sub-picosecond optical-pump electron-probe experiments will take advantage of the high electron scattering cross section to reconstruct the geometric dynamics of the valence-charge lattices. In the future, a broad class of phenomena can be addressed through this experimental approach, including the physics of stripe formation in Cuprates and other types of electronic symmetry breaking.

Pump: To date, most studies of ultrafast phase transitions have relied on the impulsive excitation of electron-hole pairs across a bandgap. This is an ultrafast analog to the process of chemical doping that explores the potential energy surface of the excited state, which is generally more difficult to model theoretically than is the electronic ground state. For this and other reasons, we are pursuing novel excitation strategies for impulsive initiation of phase transitions in the electronic ground state, such as the coherent excitation of Mn-O optic phonons with midIR pulses.

Probe: The probe for these experiments will be a femtosecond pulse of $10 \mathrm{keV}$ electrons, which will be derived from the electron gun. These ultrashort electron pulses diffract from ordered valence charges. Rearrangements in the charge-ordered patterns will be most clearly visible at wavevectors that are not commensurate with the atomic lattice.

\section{Accomplishments:}

Progress to date using FY'05 LDRD funding include: (1) design of a prototype electron gun, (2) initial testing of some critical components, and (3) preliminary optical experiments based on vibrational excitation of a phase transition in the perovskite $\operatorname{Pr}_{(1-x)} \mathrm{Ca}_{\mathrm{x}} \mathrm{MnO}_{3}$.

During FY'05, an ultrafast electron diffraction (UED) gun has been designed. This design has been refined based on initial testing using an existing x-ray streak camera as a prototype. A gold-coated sapphire disk is used as the photocathode, and the generated electron beam traverses an accelerating anode and is spatially filtered by a small pinhole. Two deflection plates are used to control the electron trajectory and also enable measurement of the electron pulse width by streaking. A magnetic lens focuses the electron beam onto the detector consisting of a micro-channel plate (MCP) and phosphor screen. The evolution of the electron pulse along the designed drift region has been simulated using the computer codes Impact $T$ and Parmelar. The spatial distribution of the magnetic field has been checked experimentally since it is the most critical design aspect of the electron gun. The good agreement between theory and experiment gives us the necessary accuracy in predicting the time of flight effects from the gun. The simulations suggest that the pulse duration of the current design is nearly 1 ps at a charge of a few thousand electrons per pulse. We have determined that the main contribution to the pulse duration is the broad energy distribution of photo-electrons, and geometric broadening due to the incident angel on the photocathode. Simulations of the new design indicate pulse broadening of only $400 \mathrm{fs}$ at a distance of $40 \mathrm{~cm}$ from the photocathode. 


\title{
Technique Development for Aberration Corrected Microscopy at NCEM
}

\author{
Principal Investigator: Ulrich Dahmen
}

\section{Project Description}

The field of electron microscopy is undergoing a revolution based on major recent advances in electron optics, detectors, stage design and computing power. These advances make it possible to overcome the fundamental limitations currently imposed by lens aberrations. The ability to correct for these aberrations is about to transform the field of electron microscopy, and the TEAM construction project, led by NCEM on behalf of five DOE microscopy efforts (LBNL, ANL, BNL, ORNL and FS-MRL at UIUC) will initiate a new generation of electron optical instrumentation. However, in the process of surpassing traditional limits of instrumental resolution and sensitivity, new barriers often rise to the surface. It has been found that such limitations are frequently posed by the sample itself, the stability of the microscope site, the sample stage and the ability to quantify experimental data for accurate comparison with atomistic simulations.

The current project will address some basic scientific and technological issues to help lay the scientific foundation and prepare for the challenges posed by this new technology. The objective is to develop techniques for imaging, spectroscopy, samples, stages, data analysis and operating parameters under aberration corrected electron microscopy. To take advantage of the greatly increased sensitivity of aberration corrected beams it will be necessary to develop new sample preparation methods using innovative techniques of ion beam thinning with novel ion sources and geometries. It is equally important to begin evaluation of prototype aberration correctors and monochromators in the context of scientific research on the atomic structure of materials. Evaluations of these novel electron optical components will need to proceed in close collaboration with their designers. This is particularly important in these first stages of their development, where the integration of both components into the standard electron optical column is by no means straightforward, and is typically optimised for only a single application. A full appraisal of the limitations and benefits of each configuration for applications to materials research on defects and nanophases will be a crucial factor in this research.

\section{Accomplishments}

One of the two VG STEM columns acquired for the project was successfully retro-fitted at NCEM with an aberration corrector. Initial tests of the updated system have highlighted the importance of the environment where such high-end machines are installed, as electrical and acoustic noise that did not affect the equipment previously installed on the site have hampered the performance of the new aberration-corrected microscope. However, thanks to improved environmental stability, recent imaging results on test gold nanoparticles have shown promise and are now almost on par with what is expected of this state-of-the-art system. Although the emphasis has so far been on the high-resolution imaging mode of the system, a high energy resolution mode for chemical analysis and a potential third hybrid mode were also identified: it was found that the optical alignment of the system could be re-optimised by an experienced user to switch between those configurations with relative ease. The versatility of the VG instrument thus seems to exceed initial expectations.

In parallel to these first steps in the aberration-free world, the portability to other systems of stage designs initially carried out for the TEAM microscope was assessed. Plans and preparatory considerations were made to replace the VG STEM stage with a more reliable and accurate TEAM-inspired stage. The second VG column was earmarked as an ideal tool for such experiments, with a view to implementing the designs in the NCEM aberration-corrected VG, making it a truly unique instrument. 
Ordering and shape control of epitaxial Ge nanostructures on metal-patterned $\mathrm{Si}$ Principal Investigator(s): Oscar D. Dubón, Jr, J. Alexander Liddle, and Daryl C. Chrzan

\section{Project Description}

The organization of nanoscale elements into higher order systems remains a fundamental challenge in nanoscience and nanotechnology. The realization of macroscopic assemblies of nanostructures through direct manipulation, achieved by highly-reproducible patterning processes such as lithography, is not only impractical but also in general unfeasible. Thus, considerable effort has been placed on the integration of spontaneous self-assembly processes that minimize the need to directly manipulate each nanoscale element but that are characterized by high defect densities. We have discovered a semiconductor growth process based on rudimentary patterned metallization that represents a natural convergence between directed pattering techniques and self-assembly processes for the realization of ordered nanostructure arrays. Specifically, we have used the superior materials deposition control afforded by molecular beam epitaxy in combination with the island site selectivity of patterned substrates to grow two-dimensional arrays of epitaxial Ge islands on Si. Nanolithographically produced stencil masks to form metal patterns on Si yield a precise template for the growth of the Ge islands while theory and modeling provide important insights into the growth process.

\section{Accomplishments}

Hundreds of thousands of Ge islands were assembled into a square lattice on a Si (001) surface that has been patterned with sub-micron Au dots. Kinetic Monte Carlo (KMC) simulations were performed to study the dynamics of nucleation, growth and coarsening on the patterned surface. These simulations elucidate a growth process in which a barrier to surface diffusing Ge atoms forms around each Au dot (Fig. 1). At a sufficiently small Au-dot spacing, islands grow into an ordered array in response to interacting diffusion barriers. Interestingly, by varying the metal species (e.g., using $\mathrm{Sn}$ rather than $\mathrm{Au}$ ) and the crytallographic orientation of the Si surface, we have realized a variety of unique island shapes including Ge nanorods (Figure 2), which may be suited for studies of low-dimensional electronic transport.

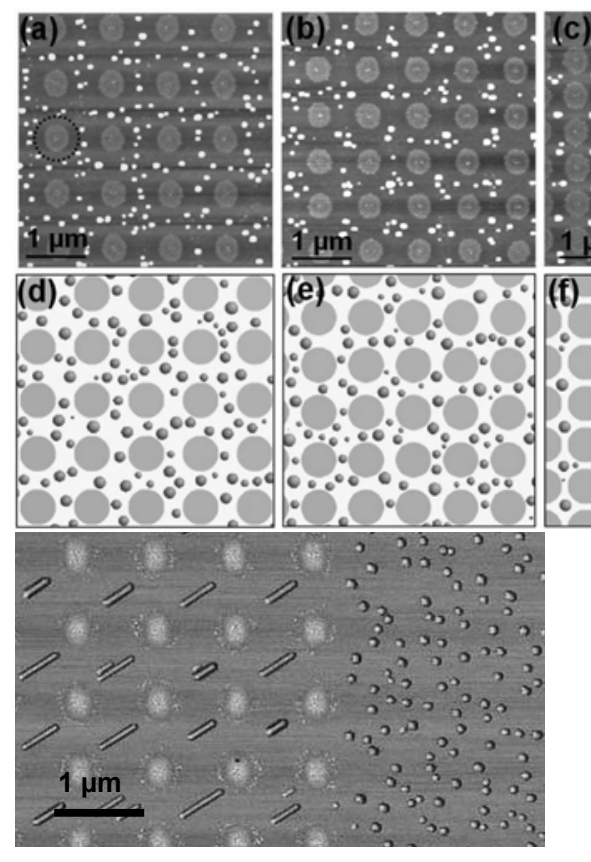

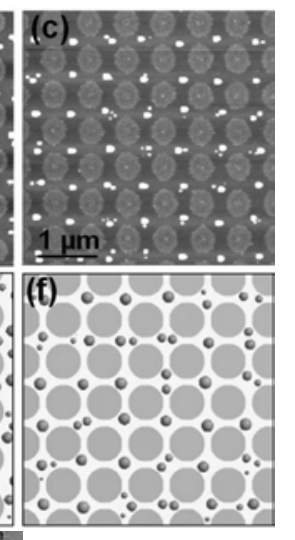

\section{.} diffisioproduce the experimental observations. Here the diffusion barriers are represented by the large, periodic gray circles. Simulations were performed on samples with proportions similar to those employed experimentally but at a smaller scale to facilitate computation.

Figure 1. Effect of Au-dot spacing on Ge island ordering observed by atomic force microscopy, $\operatorname{AFM}(\mathrm{a}, \mathrm{b}$, and c). In (a) the circular diffusion barriers (one of them indicated by the dashed circle) surrounding the Au-dots do not interact with each other, and Ge islands (small white features) grow around each Au dot. As the Au-dot spacing is reduced [(a) to (c)], Ge-island ordering occurs. The $\mathrm{z}$-scale in the AFM images is truncated at $20 \mathrm{~nm}$ to enhance viewing of the island positions relative to the much shorter Au-dots. Growth and nucleation of 3-D islands determined by KMC simulations result in the corresponding island distributions shown in (d), (e), and (1) and reproduce the experimental observations. at a smaller scale to facilitate computation.

Figure 2. AFM phase image of the boundary between a Au-patterned and a $\mathrm{Au}$-free region after $\mathrm{Ge}$ deposition on $\mathrm{Si}(110)$. The Au pattern induces the growth of ordered Ge rod-shaped islands. 


\section{Light-activated ion channels and transporters Principal Investigators: Ehud Isacoff, Dirk Trauner, Richard Kramer}

\section{Project Description}

Ion channels and transporters are proteins in the lipid membranes of all cells whose activity is normally controlled by voltage, chemicals, temperature, or mechanical force. Our LDRD proposes to create nanoscale phototransistors by modifying these ion transport proteins with novel chemical moieties that act as photo-activated "gates."

Our specific aims are: 1) to extend our photo-switched "linker-blockers" to $\mathrm{K}^{+}$channels with altered ion selectivity, and thus altered electrical effect, 2) to expand to photo-switched "linker-ligands" (linker-agonists) of receptor-channels, and 3) to create "nano-tweezers," nanometer scale force actuators that pry channels open or drive transporters to pump.

\section{Accomplishments}

1) Light-activated ion channels: Photo-isomerizable linker-blocker. The first strategy targets photo-isomerizable linker-blockers to introduced cysteine residues that are genetically engineered into suitable positions on the extracellular surface of a channel to provide for reversible light-induced block and unblock of the permeation pathway - an approach that is tantamount to the introduction of an orthogonal light-controlled gate. Our first set of photoisomerizable linker-blockers for $\mathrm{K}+$ channels have been made successfully. We recently (in December, 2004) published a description of the first of these, MAL-AZO-TEA, and the $\mathrm{K}^{+}$ channel that it controls in Nature Neuroscience (Banghart et al., 2004). We have dubbed the

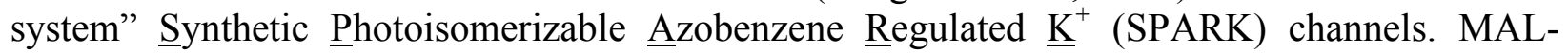
AZO-TEA, has a maleimide (MAL) group for covalent attachment to a cysteine reside, an AZO group for photo-isomerization, and a TEA group for blocking the $\mathrm{K}^{+}$channel pore. Illumination with $380 \mathrm{~nm}$ light converts the molecule from trans to cis, shortening the AZO moiety by about $7.2 \AA$ thus pulling the TEA out of reach of the pore and turning on the conductance, while illumination with $500 \mathrm{~nm}$ light photoisomerizes MAL-AZO-QA to trans, extending the linker, allowing the TEA to reach the pore and re-block it, turning the conductance off. The process can be reversed many times, rapidly and efficiently.

2) A light-activated non-selective cation channel. The ion channel protein component of SPARK channels shows high selectivity for $\mathrm{K}^{+}$over other cations, such as $\mathrm{Na}^{+}$. Therefore, opening these channels with light allows the selective flux of $\mathrm{K}^{+}$and generates hyperpolarization of membranes. To enable more diverse and accurate control of ion fluxes and membrane potential, it would be useful to engineer light-activated ion channels that have different ion selectivities. We have preliminary results demonstrating our construction of a non-selective cation channel that depolarizes membranes in response to the same wavelength of light. Our strategy entails introducing point mutations into the pore-linking region of $\mathrm{K}^{+}$-selective channels, which reduces the permeability ratio of $\mathrm{K}^{+} / \mathrm{Na}^{+}$from $\sim 1000$ to $\sim 1.4$. Expression of these channels in cultured cell lines results in the appearance of a light-activated conductance that can depolarize cells, and in hippocampal neurons, the depolarization is sufficiently large to trigger action potentials. 
Nanoscale Lithography to Guide Self-Assembly for the Creation of Functional, Hierarchical Nanostructures

Principal Investigator(s): J.Alexander Liddle and Nitash P. Balsara

\section{Project Description}

Length scales that are accessible with conventional lithographic techniques are limited to approximately $25 \mathrm{~nm}$ - much larger than many potentially useful structures such as nanotubes, nanocrystals or functional molecules. A method of integration is required to allow the imposition of a structural hierarchy and interconnection between the various elements in order to realize the full potential of many nanoscale objects. Guided self-assembly provides a means of addressing this issue. The goal of this research is to develop a "toolkit" based on lithographically guided self-assembly suitable for bridging the gap between current lithographic techniques and the nanoscale.

We have chosen to use diblock copolymers, systems that already exhibit well ordered microstructures, as the starting point for our research. Not only do these materials allow a very large degree of latitude in terms of the chemical functionality, which can be added before or after assembly, but they also provide an interesting window into the soft-hard matter interface.

The research addresses simultaneously the areas of lithography and materials - a deliberately synergistic approach - in order to make the most rapid progress. We have concentrated on two model systems. First, we have used a poly(styrene-b-isoprene) diblock copolymer - a relatively well understood system - that enables us to explore the fundamental mechanics of the assembly process. Second, we have synthesized poly(styrene- $b$-isoprene- $b$-ethylene oxide) triblock copolymers that will enable us to investigate the potential for sub-lithographic pattening.

\section{Accomplishments}

We have successfully used high-resolution electron-beam lithography to generate chemically patterned surfaces to control the assembly of a lamellar poly(styrene-b-isoprene) diblock. We have used the flexibility afforded to us by this direct-write patterning process to generate, for the first time, polymer microstructures that are far from the equilibrium morpohology. This has enabled us to examine the trade-off between surface and bending energies in controlling the polymer structure, and gives us a tool to probe the elastic behavior of these materials. We have managed to create structures in which the minimum radius of curvature is equal to one quarter of the domain spacing of the diblock (a radius of curvature of $12 \mathrm{~nm}$ was achieved in a diblock with a $48 \mathrm{~nm}$ domain spacing).

Currently the assembly process has a yield of $60 \%-70 \%$. We are endeavoring to understand what controls this and to develop more robust methods with a view to having processes that can eventually be used in a manufacturing environment.

We have also begun to investigate the ability of the self-assembly process to repair defects in the original lithography. Even though characteristics of the chemically patterned surface such as line edge roughness are very poor, the diblock material generates very smooth, uniform structures following the assembly process. This is potentially an extremely powerful method to achieve a degree of perfection in structures at the nanoscale that cannot be realized in any other way. 


\section{Science and Technology of Quantum Materials}

Principal Investigators: J. W. Orenstein; R. Ramesh; D-H. Lee; J.E. Moore; Y. Suzuki;

Collaborators: R. Birgeneau (UCB); N. Browning (LLNL); D.C. Chrzan; A. Lanzara; A. Vishwanath Project Description:

"Quantum materials,"systems in which the quantum-mechanical correlations of electrons play a dominant role, constitute a rich and diverse palette of physical phenomena. In such materials interacting charge, spin, orbital, and lattice degrees of freedom allow for a broader range of phase space, exemplified in the perovskite manganites, cuprates, ruthenates and cobaltates. At LBNL, we have brought together an interdisciplinary team of researchers, including theorists (Lee, Vishwanath, Moore) thin-film synthesis (Ramesh and Suzuki), and characterization (Browning, Birgeneau, Hellman, Lanzara, and Orenstein). Our LDRD program is probing novel quantum phases and their phase transitions in carefully prepared single crystals and comparing/contrasting these observations to those in epitaxial thin film heterostructures. This LDRD forms the backdrop for a comprehensive proposal to DOE that has already been submitted for review.

Accomplishments:

We have probed the magnetism and transport in epitaxial $\mathrm{La}_{1.2} \mathrm{Sr}_{1.8} \mathrm{Mn}_{2} \mathrm{O}_{7}$ thin films synthesized in a wide range of deposition conditions. With the (110) orientation, the $\mathrm{La}_{1-\mathrm{x}} \mathrm{Sr}_{\mathrm{x}} \mathrm{MnO}_{3}$ bilayers lie perpendicular to the substrate plane with the $c$-axis in the plane of the film, allowing us to measure the magnetic and electronic anisotropy of the material in a single sample. The thicker films possess bulk-like properties with a ferromagnetic transition that coincides with a resistivity maximum at a reduced $T_{c} \sim 90 \mathrm{~K}$ (vs. $120 \mathrm{~K}$ in bulk) and an anisotropy ratio, $\rho_{c} / \rho_{a b} \sim 20$-200 over the range of 5-380 K.

Orenstein and Ramesh are collaborating on understanding spin dynamics in a model correlated system, SrRuO3. In epitaxial thin films, the optical measurements show the existence of a clear ferromagnetic resonance at a relatively high frequency of $\sim 250 \mathrm{GHz}$. Further studies are underway to explore this phenomenon fully and its implications for spin torque experiments. The collaboration between Ramesh, Suzuki and Orenstein is focused on exploring new classes of correlated materials with the potential for multiferroic behavior, such as vanadium-based perovskites. We have grown single phase, epitaxial thin films of lead vanadate $\left(\mathrm{PbVO}_{3}\right)$ using pulsed laser deposition. Structural analysis of the $\mathrm{PbVO}_{3}$ thin films using transmission electron microscopy (NCEM), XRD and RBS reveal that the films are single tetragonal phase, with $a=3.79 \AA$ and $c=5.02 \AA(\mathrm{c} / \mathrm{a}=1.32)$. Electron energy loss spectroscopy(NCEM) and x-ray absorption spectroscopy(ALS) were used to show the stabilization of the $\mathrm{V}^{4+}$ state, thereby proving the creation of a new $d^{l}$ system for intensive physical study. Studies of the magnetism phase transitions and transport in $\mathrm{PbVO}_{3}$ are underway.

The theoretical work of Moore and Vishwanath addresses electrical and thermal transport at a two-dimensional quantum phase transition into a superconducting phase. The relevance of this work to the new two-dimensional electron systems to be created as part of this LDRD is threefold. First, it required development of new numerical and analytical methods in order to access thermal and electric transport properties of a quantum critical electronic system. Second, the particular transitions studied here may be realized in doped Mott insulator heterostructures (see work above). Finally, this project highlighted the importance of thermoelectric and thermal transport as a tool to studying correlated systems and laid out the formalism for calculating these in other situations. Work is currently focused on modeling the correlated two-dimensional electron system using adaptations of standard correlated electron models to oxide nanostructures. Lee has initiated work with postdoc Ribeiro on the bilayer Hubbard model where one layer is n-type doped and the other is p-type doped. The intention is to model the self-doping of two Mott insulators at a interface. The results show that when the inter-layer electronhole Coulomb attraction is sufficiently strong, an interface exciton is formed. When that happens, the bilayer behaves as a Mott insulator together, however each member of the bilayer can still be superconducting or Fermi liquid individually. The detail phase diagram is currently being studied. 
Computational and theoretical studies of bulk and nano solid systems Principal Investigator(s): Ivo Souza and Steven G. Louie

\section{Project Description}

The purpose of this project is to develop efficient techniques for sampling the ab-initio Fermi surface in electronic structure calculations of metals. This will reduce dramatically the computational cost of calculations of several key properties of metals, such as transport coefficients and electronphonon coupling. Presently such calculations require access to supercomputers. With the proposed method they will become feasible on a laptop computer. This is expected to broaden dramatically the range of problems that can be treated by first-principles methods.

The problem that motivated this work is the so-called anomalous Hall effect in ferromagnets, whereby a transverse Hall voltage develops when a current is passing through a magnetic conductor, even in the absence of a crossed magnetic field, which is required in the usual (classical) Hall effect. Recently, a first-principles calculation of the anomalous Hall conductivity (AHC) of iron as a Brillouin-zone integral of the Berry curvature was carried out and found to be in reasonable agreement with experimental results. However, the authors observed extraordinarily strong and rapid variations of the Berry curvature wth wavevector $k$ in the vicinity of avoided crossings and near-degeneracies in reciprocal space. A conventional first-principles calculation thus requires an extremely dense $k$-point mesh and is quite time-consuming. Our new approach for computing the AHC is based on Wannier interpolation. First, a conventional electronic-structure calculation is performed for iron, with spin-orbit included, on a relatively coarse $k$-point mesh. Second, maximally-localized Wannier functions are constructed by a post-processing step, thus transforming the full ab-initio problem into an effective tight-binding form. Finally, the needed quantities such as Berry potentials and curvaturesare interpolated onto a fine $k$-point mesh and used to compute the AHC.

Accomplishments

Preliminary results indicate that our approach gives good agreement with conventional, less efficient first-principles calculations of the AHC of iron. We have also used this technique to evaluate other tranport properties as Fermi surface integrals, namely the low-field classical Hall conductivity, again finding very good agreement with previous, rather more expensive calculations. based on our calculations, we are currently developing new insights into the mechanisms of the AHE, by breaking it down into separate contributions from different parts of the Fermi surface. Of particular interest are the regions where spin-orbit coupling lifts the degeneracy between spin-up and spin-down Fermi surface sheets.

A parallel development is the application of the Wannier interpolation technique to the evaluation of electron-phonon coupling, a key quantity in the first-principles modelling of superconductivity, for example. 


\section{COMPLEX OXIDE NANOSTRUCTURES: \\ Understanding Surface, Interface and Finite Size Effects through Nanofabrication \\ Yuri Suzuki (PI), Alex Liddle, (co-PI), Andreas Scholl (co-PI) and Hendrik Ohldag}

\section{PROJECT DESCRIPTION}

In this program, our major objective has been to develop nanofabrication techniques for complex oxide materials in order to (i) develop a fundamental understanding of the important length scales and the role of surfaces and interfaces in the magnetism of highly spin polarized materials and more broadly to (ii) enable the studies of other classes of complex oxides at nanometer length scales. Through direct electron beam patterning, we have probed the relationship among structure, surfaces and magnetism in geometrically confined islands of doped perovskite manganites.

One of the biggest challenges in studying the behavior of complex oxide materials at the nanometer length scales has been fabrication of stoichiometric nanoscale samples. Complex oxides offer a wide range of functional properties. We can tune these functionalities by cation distribution, strain as well as geometrical confinement. In particular, understanding the role of surfaces and interfaces is key to the ultimate success of these functional oxides. Among the complex oxides, ferromagnetic oxides in which the carriers are highly spin polarized have received much recent attention. In these materials, the spin of the electron is used to augment its charge to provide enhanced functionality. This added functionality due to the spin degree of freedom may potentially facilitate decreased electrical power consumption, nonvolatility, increased integration densities and increased processing speeds in a wide range of electronic applications.

\section{ACCOMPLISHMENTS}

We have successfully developed a novel technique to define nanometer scale islands of complex oxide thin film materials. The technique involves electron beam patterning followed by Ar+ ion beam implantation to selectively eliminate the magnetism in the sample. Since this technique does not involve any etching, the resulting samples have a flat profile which is crucial for magnetically imaging the islands by photoemission electron microscopy (PEEM).

The samples consist of $60 \mathrm{~nm}$ thick epitaxial thin films deposited on (001)- and (110)oriented $\mathrm{SrTiO}_{3}$ substrates by pulsed laser deposition. The choice of substrate orientation results in thin films with two- and four-fold in-plane anisotropy, respectively. A $500 \mathrm{~nm}$ thick resist pattern is used to define regions of varying shape and size that are/are not exposed to an $\mathrm{Ar}^{+}$ion implantation at $100 \mathrm{keV}$ with a dose of $1.0 \times 10^{14}$ ions $/ \mathrm{cm}^{2}$. By disrupting the ordering of the lattice, the implanted ions cause the degradation of the magnetic and transport properties of the
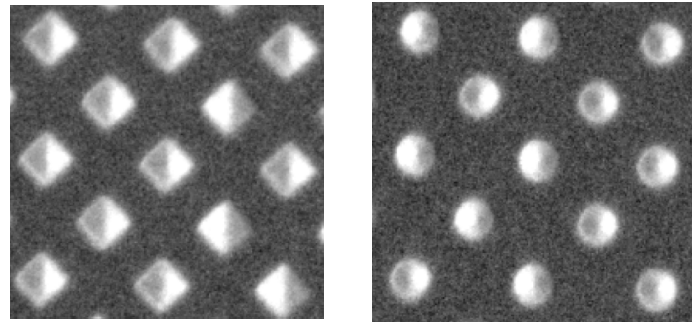

Figure 1. Magnetic contrast images of diamonds $500 \mathrm{~nm}$ on a side and $500 \mathrm{~nm}$ in diameter indicate multidomain states that suggest that shape anisotropy effects dominate magnetocrystalline anisotropy effects. doped mangnaite $\mathrm{La}_{0.7} \mathrm{Sr}_{0.3} \mathrm{MnO}_{3}$ (LSMO) film. PEEM and magnetic force microscopy (MFM) images reveal that the domain structure in the islands depends predominantly on the shape of the islands, with the magnetocrystalline and magnetoelastic energies contributing secondary effects.

Moreover, we have successfully synthesized the bilayer manganite $\mathrm{La}_{1.2} \mathrm{Sr}_{1.8} \mathrm{Mn}_{2} \mathrm{O}_{7}$ with the ferromagnetic layers perpendicular to the substrate. This geometry enables us to probe the magnetic and transport anisotropy in these materials. 
Experimental Signatures of Fractionalized Phases and Phase Transitions in Strongly Correlated Electronic Systems

P.I. : ASHVIN VISHWANATH

\section{Project Description}

The main focus of this project was to understand some of the conditions under which strongly interacting many-particle quantum systems are described in terms of fractionalized excitations (excitations that carry fractional quantum numbers) and the resulting experimental signatures. Recently, there has been an improved understanding of the universal features of such states (for example they are necessarily accompanied by an emergent gauge theory in its deconfined phase), both as stable phases and as quantum critical points. However the microscopic conditions under which these states may arise, and also their characteristic experimental fingerprints are less well understood and this was sought to be remedied by the present study. Ultimately, it is hoped that with a better understanding of these issues will help answer the question of which strongly correlated materials controlled by this interesting new physics.

Accomplishments

The question of microscopic conditions under which the phenomenon of fractionalization can occur was addressed by considering quantum lattice models which may be studied in a rigorous way theoretically and could also be realized in cold atom experimental setups or frustrated insulating magnets. In particular we were interested in realizing a microscopic model that displays deconfined criticality - a continuous quantum phase transition at which fractionalized degrees of freedom emerge right at the critical point, but are bordered by conventional confined phases on either side. A promising candidate was provided by hard core bosons on the triangular lattice where superfluid and solid phases were expected, and the direct continuous transition between them was a candidate deconfined quantum critical point. However, much to our surprise, the model with nearest neighbor repulsion at half filling is a superfluid even with large repulsion, a consequence of the frustrated nature of the triangular lattice Ising model. Thus, instead of deconfined criticality, we found a persistent supersolid phase. While obtaining a supersolid phase was not the initial motivation, it was still a very interesting outcome since this was the first observation of a robust supersolid phase in a microscopic model of bosons. Moreover, this gave us insight into the conditions when deconfined criticality is pre-empted in this manner. The results were presented in Physical Review Letters. In a separate study the experimental signatures of fractionalized excitations in dilute system of cold atoms was studied. We considered the Luttinger liquid state of a one dimensional interacting Fermi gas, which also displays fractionalization, and asked how such a system may be probed. Of particular interest are the values of the Luttinger parameters. We found that noise correlations could provide this information, and these results have been written up in the form of a preprint. In a related project, Arun Paramekanti, the postdoc supported by the LDRD grant, studied the properties of the undoped cuprate antiferromagnet with ring exchange interactions, using variables "Schwinger Bosons" that are appropriate if the system is in proximity to a deconfined quantum critical point or phase. 


\title{
Nuclear Science Division
}

LB04017

\author{
Research and Development for Double Beta Decay Experiments \\ Principal Investigator: Kevin Lesko
}

Project Description

We are faced with several fundamental questions about neutrinos: their absolute mass, the ordering of the neutrino masses, and the prospect of determining if the neutrino is its own antiparticle. Neutrinoless double beta decay experiments can deliver evidence leading to the determination of the absolute neutrino mass; they can determine the hierarchy of the neutrino masses, and, most importantly, they provide the only technique to determine if the neutrino is Dirac or Majorana in nature. Recently the DOE and the NSF convened a committee (NUSAG) to review and assess the scientific case for neutrinoless double beta decay and several other neutrino experiments; both Majorana and CUORE were approved for the next stage of review.

The purpose of this project is to develop key technologies, data analysis tools, and comprehensive simulation packages essential for the success of next generation neutrinoless double beta decay (0vDBD) experiments. Among the several candidates for the next generation of experiments are Majorana, using Ge-diodes, and CUORE, using $\mathrm{TeO}_{2}$ cryogenic crystals. Radioactive backgrounds are the major obstacle for both these experiments. The Ge experiment draws on nearly four decades of experience operating Ge-diodes. The Te cryogenic technique has made great progress in developing and operating arrays of $\mathrm{TeO}_{2}$ crystals. Controlling, understanding and identifying backgrounds in both techniques remain the greatest challenges for this field. A variety of topics has been undertaken to achieve this goal of reducing the sensitivity of the experiments to backgrounds through the physical reduction of the radioactive backgrounds, by investigating detector designs, identifying background signals, and by developing simulation and analysis packages.

Progress and Accomplishments

For the Majorana collaboration we are developing and maintaining the comprehensive Monte Carlo simulation package. Our efforts have focused on using modern simulation tools from the high energy and nuclear physics communities to assemble an experimental simulation package that can be shared with the entire collaboration. Using modern languages and architecture we released the verified package in 2005. This package handles the detector geometries, media properties, shielding characteristics, and physics interactions. We continue to work with GRETINA, to develop segmentation schemes and data analysis to reduce the sensitivity to backgrounds. Berkeley coordinates the Low Background Counting efforts. Berkeley is responsible for the design and fabrication of the germanium detector. In this role we have begun estimating the costs and risks associated with different detector designs and researching vendors and suppliers for the detector fabrication. We are investigating front-end electronics designs. All these efforts will be used in the proposal for the Majorana project to be submitted in early 2006.

For the CUORE project we are developing data analysis techniques and building a US analysis program for Cuoricino, refining the production procedure for thermistors, and investigating $\mathrm{TeO}_{2}$ crystal production with minimal contamination. The US data analysis program will be in routine operation in mid-2006. Thermistor production saw significant progress in understanding the neutron dose needed to create the thermistors. We are pursuing vendors for $\mathrm{TeO}_{2}$ crystals and investigating crystal bonding techniques. The research into backgrounds has yielded some advances in understanding the origin of the background signals observed in the Cuoricino prototype; this holds promise for controlling these in the proposed CUORE experiment. 
Establishing New Capabilities in Nuclear Astrophysics and Radiation Biology using Neutrons at the 88-Inch Cyclotron

Principal Investigator: Margaret A. McMahan

Project Description

This project is to apply new and recent neutron, radioactive beam and radioactive target capabilities at the 88-Inch Cyclotron to studies in s-process nuclear astrophysics and radiation biology with neutrons. A new beam line at the 88-Inch Cyclotron will produce quasimonoenergetic neutron beams in the range of $10-30 \mathrm{MeV}$. This project is to extend the new neutron capability to lower energies $(<200 \mathrm{keV})$. It will also demonstrate the feasibility to measure new neutron capture cross sections on radioactive targets by measuring the ${ }^{85} \mathrm{~K}(\mathrm{n}$, gamma) cross section, an important branch point nucleus in s-process models. It will also characterize the neutron beam and design collimators and a position-sensitive transmission ion chamber for radiation biology. These research directions are well aligned with programs of national importance such as RIA and new space exploration of the Moon and Mars. These basic science measurements are also important inputs for some of the stockpile stewardship codes in use at the NNSA laboratories.

There are many other uses for a neutron beam - either quasi-monoenergetic or with a broad energy (white) spectrum - in both basic science and applications; most of these require either further beam development or instrumentation which was beyond the scope of the original funding. Hence the present project, for which two milestones were set for Year 1, one for each of the two new programs:

1) For s-process nuclear astrophysics. Designing a lithium target and characterizing the flux, energy and angular distributions for low energy neutron production using the ${ }^{7} \mathrm{Li}(\mathrm{p}, \mathrm{n})$ reaction. This is to demonstrate the feasibility of the proposed cross- section measurement of neutron capture on radioactive targets.

2) For radiation biology. Design and build a position-sensitive transmission ion chamber useful for characterizing all neutron beams and for providing dosimetry for radiation biology and soft error rate testing.

\section{Accomplishments}

Our first proposed milestone has been put on hold, because the existing (LLNL) radioactive ${ }^{85} \mathrm{Kr}$ target with which we proposed to do this measurement was determined to be too contaminated to be useful. Therefore we have spent our effort looking at the feasibility of producing highly pure $\mathrm{Kr}$ targets using the 88-Inch Cyclotron for neutron capture experiments. While a measurement of this reaction is very important to understand s-process nucleosynthesis, the experiment is extremely difficult because the final product to be measured is stable. In order for the experiment to work, this gas target must be produced and transferred to the neutron beamline with virtually no air leaks. We are still examining ways to accomplish this in Year 2 of the project.

A prototype position- sensitive transmission ion chamber has been designed using a $\mathrm{ZnS}(\mathrm{Ag})$ scintillating layer - which is sensitive to fast neutrons but not to gamma rays - which will be backed by a photocathode layer and then gaseous electron multiplier layers. The $\mathrm{ZnS}$ is being tested now with a standard photomultiplier tube, the prototype transmission counter is under construction and the electronics are being designed. 


\section{Improved Spectroscopy of Weakly Bound States in Nuclei Principal Investigator: Larry Phair}

\section{Project Description}

Future nuclear structure measurements will take place at the drip lines of very neutron-rich and proton-rich nuclei. These nuclei will be identified by detecting the last particle-stable isotope and the spin and parity of its ground state and low excited states, if any. By studying states at high excitation energy now (i.e., the unpopulated drip-line states), we can explore in a novel way the single particle, pairing, and vibrational properties of states more commonly studied near the Fermi surface in ordinary nuclei.

Our goal is to demonstrate the feasibility of studying the future drip-line single particle and collective states by populating these states which lie about 6-7 MeV above the Fermi surface in ordinary nuclei. If this technique works, a program could be implemented to study drip-line states via direct reactions and gamma-ray measurements using the high efficiency GRETINA array.

\section{Accomplishments}

Within the first six months of the year we installed and commissioned an array of gamma-ray and charged-particle detectors on a dedicated beam line at the 88-Inch Cyclotron. Presently, the array comprises 6 "clover-type" germanium gamma-ray detectors and a silicon charged-particle detector (called STARS), complete with about 150 channels of electronics and a new data acquisition system. This equipment is now fully operational and the first experiments have recently been performed.

Direct reactions have been measured using the light ion beams from the 88-Inch Cyclotron. The reaction ${ }^{208} \mathrm{~Pb}(d, p){ }^{209} \mathrm{~Pb}$ was performed at two beam energies to populate single particle states in ${ }^{209} \mathrm{~Pb}$ both near the surface and at higher excitation energies as well. States near the surface were identified cleanly with both the charged particles and the coincident gamma rays. We will use the gamma rays detected with high resolution in the Ge crystals to characterize the neutron states at high excitation. The detected charged particle will be used to establish the excitation energy of the nucleus, while the coincident cascade of gamma rays will be used to establish the structure of weakly bound states, e.g., the single particle strength function of populated states. High efficiency coincident gamma rays have been employed for the first time ever in studying direct reactions with these measurements.

In the second experiment called for in the LDRD, a beam of ${ }^{17} \mathrm{O}$ at $380 \mathrm{MeV}$ was used to excite a ${ }^{208} \mathrm{~Pb}$ target. The heavy ions were used to inelastically excite collective states in ${ }^{208} \mathrm{~Pb}$. The scattered ${ }^{17} \mathrm{O}$ was detected in the STARS array and the gamma rays de-exciting high-lying states in ${ }^{208} \mathrm{~Pb}$ were measured in the Clover Ge detectors. Previous experiments used low-resolution gamma-ray detectors $\left(\mathrm{NaI}\right.$ and $\left.\mathrm{BaF}_{2}\right)$ and while resonances, in particular the pygmy dipole resonance, were excited, no detailed structure was seen in their gamma rays. We have used the high-resolution Ge detectors to attempt to resolve individual states up to and beyond the pygmy region and to establish the decay pathways of these states.

Data analysis is near completion for both measurements. 


\title{
Physical Biosciences Division
}

LB05006

\section{Organization of Cellular Components on Signaling and Function: Theory and Computation as Essential Complements to Genetic and Biochemical Experiments}

\author{
A.K. Chakraborty, A. Arkin, P. Collela, P. Geissler, T. Head-Gordon
}

\section{Project Description}

Cell signaling involves a multitude of interacting components, whose organization into specific spatial patterns is a central regulatory motif in cell signaling. The primary aim of this project is to develop theoretical models and algorithms that will enable discoveries clarifying the principles that govern the way in which spatial patterning of components influences cellular response to external stimuli. This project will bring together advances in experimental cell biology and the computational study of complex systems to impact scientific discoveries in the twenty first century.

Accomplishments

*Methods for multiscale modeling of cell signalling (Arkin/Collela). We developed a new algorithm for efficient simulation of diffusion on complex geometric surfaces derived from three-dimensional image data of cells, which is second-order accurate in space and time. The method will be important for simulating how cell polarization or protein localization affects controllability and temporal response of signal transduction pathways.

* Monte Carlo and Langevin algorithms for spatially heterogeneous systems (Chakraborty). We have developed simulation methods over large (millimeter) lengthscales and long (hours) timescales to describe how T cells hunt for antigen presenting cells (APCs). Recent experiments using in vivo two-photon microscopy has shown that the trajectories of migrating $\mathrm{T}$ cells are characterized by a linear dependence of the squared and averaged displacement with time, leading to much debate about the role of chemokine gradients. Based on time correlation functions derived from our simulations, we find that higher order correlation functions can predict when chemokine gradients due to activated APCs are important, which has recently been confirmed by data from three experimental laboratories.

*Cell scale model of cytoskeletal dynamics (Geissler). We developed a computational model for cross-linked networks of semiflexible polymers; when applied to cytoskeletal mechanics, we found that equilibrium elastic properties of randomly cross-linked networks based on either the response of a single chain or treating the network's connections as Hookean springs, are insufficient to capture the qualitative dependence of elastic moduli on cross-link density. When cross-links are sparse, the spatial distribution of strain within the network can be highly nonuniform and can fluctuate significantly over time. Living systems might well exploit this behavior, since it generates a unique sensitivity to patterns of external stress.

* Molecular models of protein complexes (Head-Gordon). We developed a new general analytical solution for computing the screened electrostatic interaction between arbitrary numbers of proteins of arbitrarily complex charge distributions, assuming they are well described by spherical low dielectric cavities in a higher dielectric salty medium. By exploiting multipole expansion theory for the screened Coulomb potential, we describe direct charge-charge interactions and all higher-order cavity polarization correctly at all separation distances. Our solution is efficient to compute and can provide on-the-fly updates in charge distributions due to protein conformational changes. We currently are reformulating these electrostatic expressions to smoothly increase spatial resolution back to greater molecular detail of the dielectric boundaries. 
The Statistical Dynamics of Protein Evolution

Principle Investigators(s): Gavin E. Crooks

\section{Project Description}

The goal of this project is to create the next-generation algorithms for the automatic reconstruction of protein evolution from protein sequences. This requires a mathematically elegant, computational efficient and biological realistic model of protein sequence evolution over long time scales; an efficient Monte-Carlo algorithm for sampling from the posterior distribution of protein histories; and reliable benchmarks to validate the authenticity of the reconstructed histories. Three particular deficiently with existing algorithms will be addressed; the simulations inferences of sequence alignment and protein evolution using a consistent algorithm; the higher level evolution of proteins through domain reorganization; and the computational challenges that must be faced when considering the vast and rapidly growing size of the protein sequence database.

\section{Accomplishments}

We have investigated in detail a recently proposed non-Markovian model of amino acid substitution. This model persistent amino acid correlations due to persistent, heterogeneous background distributions is conceptually simple,and provides a practical and explicit description of protein sequence evolution over very long time scales. We have obtained additional evidence for this model by careful analysis of extant protein sequences and by studying computer simulations of model proteins under mutation and selection. We have also removed the major practical difficulty, namely that directly applying this model of evolution to multiple sequence alignment would be computationally expensive. This was achieved through a carefully crafted approximation. Unexpectedly, the result is similar to heuristics that have previously been employed in multiple sequence alignment. Therefore, we can now conceptually unify several existing, apparently divergent approach to the problem of multiple sequence alignment (despite superficial differences, they are approximations of one another), while also placing existing heuristics on a firm foundation. 


\title{
High-throughput Production of Proteins and Protein Complexes
}

\author{
Thomas Earnest, Physical Biosciences Division
}

\section{Project Description}

This proposal initiated a program in technology development for the production of proteins and protein complexes in the quantities necessary to pursue biological studies on these samples. This will include the expression of single polypeptide chains along with the coexpression of protein-protein complexes when biologically relevant interacting partners are identified, as well as isolating samples expressed in natural abundances using affinity-tags on members of protein complexes. The automation of this process will be pursued in order to provide a high-throughput platform for systematic biological studies. Integral membrane proteins will be attempted once the technology for soluble proteins is in place and made adaptable to the added difficulty of selecting expression systems and purification methods optimal for automating the production of this challenging set of proteins. Lastly we will explore the use of artificial intelligence methods to analyze and optimally control these experiments and the integration of this information into large-scale biological research efforts. Initial target samples will include proteins and protein complexes from prokaryotic (Caulobacter crescentus) and eukaryotic (Saccharomyces cerevisiae, Drosophila melanogaster) sources. To attempt a genomic-scale production of proteins and protein complexes, methods and technologies need to be developed that allow for the determination of which constructs and sets of constructs will produce large amounts of protein in the soluble fraction for purification, or can be refolded. Searching for the correct constructs or sets of constructs would benefit greatly from the development of parallelized screening methodologies and their implementation into an automated and intelligently controlled instrumentation environment.

\section{Accomplishments}

The identification of conditions for overexpression in these systems is optimized by parallel insertion into numerous expression vectors in $E$. coli, yeast, and baculovirus-infected insect cell lines. A procedure has been developed and automated for parallelizing attempts to achieve higher levels of expression by making several constructs with differing lengths and end residues on the $\mathrm{N}$ - and $\mathrm{C}$-terminals, utilizing green fluorescent protein as a fusion at the $\mathrm{C}$-terminal of the protein of interest as a marker for expression and solubility. We have implemented these methods on existing laboratory robots as part of this project, such as the TECAN Genesis liquid-handling robot and HIGRO multichamber incubator/shaker. In future effort we will extend this to more optimal instrumentation that can be well integrated into a production pipeline, while maintaining the flexibility required for the range of samples to be produced. Other approaches, such as the modulation of expression through RNA secondary structure, will also be attempted and implemented in a high-throughput condition search robot. Our initial prokaryotic targets are from Caulobacter crescentus, a microbial system that has promise as a bioremediation agent. Saccharomyces and Drosophila will be the eukaryotic organisms that we will initially study. Drosophila has been well characterized in particular in regard to developmental pathways. These organisms have sequenced genomes and are well studied by a variety of genetic and biochemical methods, thus providing a basis for biological and cellular understanding of the roles these proteins and complexes mediate. Recently the DOE Genomes-to-Life program has funded the effort to implement high-throughput production of multi-protein complexes through a Stanfordbased collaboration (Harley McAdams, PI). 


\section{Synthetic Cytoskeleton: Protrusive Structures for Reconstituted Cell Motility Principal Investigator: Daniel A. Fletcher}

Project Description

The purpose of this project is to develop an experimental system for studying how the actin cytoskeleton contributes to crawling motility. Crawling motility is a fundamental cellular process essential for wound healing, immune response, and embryonic development. Synthetic crawling motility could have important applications for directed drug delivery in the body, pathogen detection, and environmental sensing, but a fundamental understanding of the cytoskeletal dynamics required for directed movement is lacking. This project aims to functionally reconstitute the first step of crawling motility - protrusion - using only purified cytoskeletal proteins in lipid vesicles.

Protrusive cytoskeletal structures will be reconstituted from a simplified system of purified proteins consisting of actin and associated nucleating, branching, and cross-linking proteins. In the first year, we will characterize protrusion force and velocity of the simplified system in vitro using an atomic force microscopy (AFM) technique that we recently developed. We will then investigate spatial organization and growth of protrusive structures in lipid vesicles by initiating actin polymerization through photolysis of caged molecules. In the second year, force distribution and growth rate of the synthetic cytoskeleton will be quantified and related to the concentration and spatial organization of molecular components. This work will lay a foundation for future efforts to rebuild the entire process of crawling motility and put synthetic directed movements to use for health and technology applications.

\section{Accomplishments}

Our major accomplishments in this first year have been (1) direct measurement of force and velocity of a growing actin network in vitro and (2) development and demonstration of a caged actin system. Direct measurement of actin dynamics was accomplished using a modified AFM that enables differential measurement of actin network growth with nanometer-scale accuracy over several hours. By quantifying both force (proportional to absolute displacement) and velocity (rate of change of displacement), we were able to observe two unexpected features of the actin force-velocity relationship. First, actin growth rate is insensitive to opposing force over nearly half of its force range, indicating that small perturbations in the force opposing actinbased protrusion will not alter the rate of protrusion. Second, the velocity of actin network growth at any point in time depends not only on the instantaneous load but also on the loading history, indicating that there is no single force-velocity curve which defines actin dynamics.

The second major accomplishment this year has been development of a caged actin system that enables controlled release of actin monomers. We have demonstrated that caged actin can be photo-released locally to control elongation of actin filaments in vitro. This process was adapted to a conventional epi-fluorescence microscope and allows visualization of individual actin filaments. Future work will extend this work to encapsulation of proteins in lipid vesicles. 


\title{
LDRD: Rapid Characterization of microbial RNAs using Artificial Nanopores
}

\author{
Jan T. Liphardt
}

RNA molecules have numerous structural and functional roles in microbial molecular machines, including intron excision in T. Thermophila, tRNA maturation, and the catalysis of the peptide bond in the ribosome. RNA is also an active component of viral machines such as the rotary motor of the $\phi 29$ bacteriophage. To understand how RNA catalyses reactions and regulates biological processes, it is essential to understand the mechanical properties of large RNA assemblies, and to learn how RNA structure (e.g. helices, loops, $\mathrm{Mg}^{2+}$ binding sites) relates to RNA function (e.g. conversion of chemical potential stored in ATP to mechanical forces, catalysis of RNA cleavage and ligation).

We are developing a fundamentally new approach for determining RNA structure, dynamics, and responses to mechanical force that promises high throughput ( 1 genome per day) coarse-grained characterization of a microbe's repertoire of RNA structures. Briefly, the objective of the proposed research is to characterize the properties of biopolymers such as DNA and RNA in confined geometries using a new approach, the mechanical translocation of polymers through nanopores. If such an experimental geometry could be realized, numerous biological and polymer physical experiments would become possible.

We have now been able to obtain preliminary results showing that this approach is realizable, and that biopolymers can indeed be
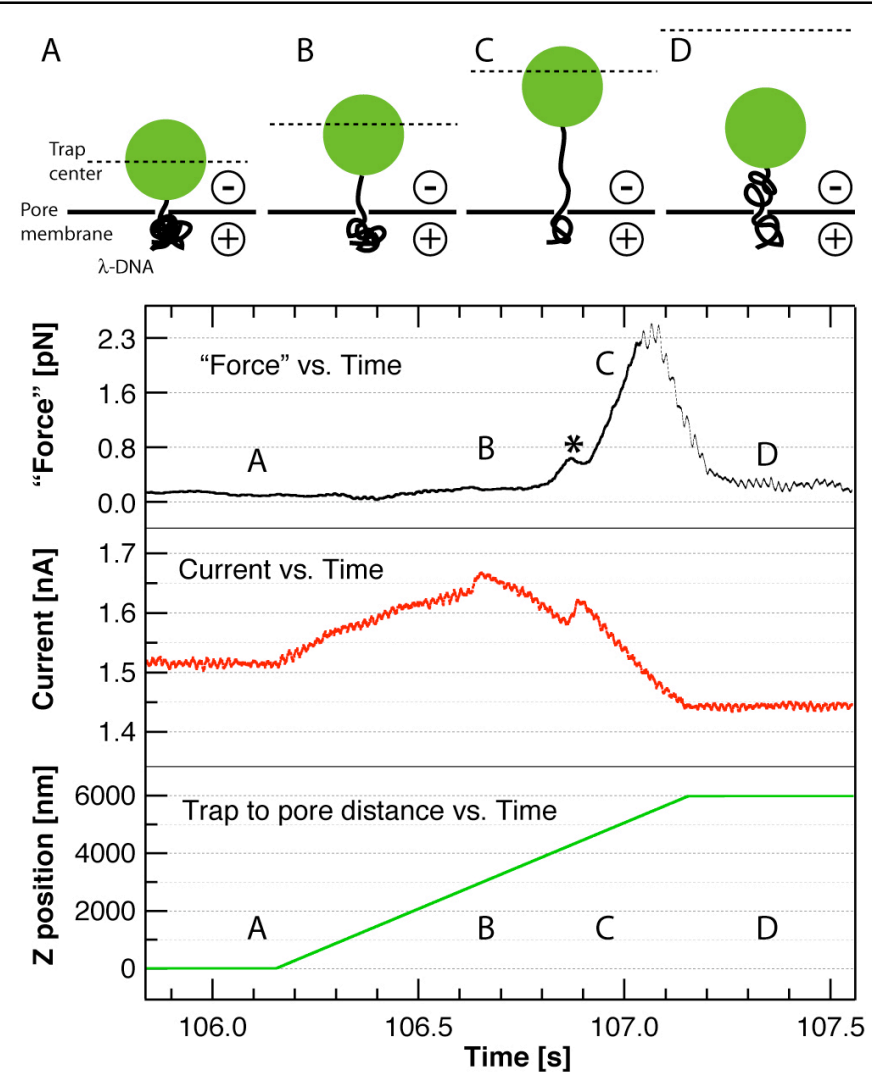

Figure 1. Stretching of a single DNA molecule by mechanical pulling through a nanopore. DNA is a highly nonlinear spring: the black curve shows the characteristic initially slow increase of force with extension (region B), and then the increasingly faster increase of the force with extension (region $\mathrm{C}$ ). A "rip" is also observed (star), indicating a molecular stick-slip event.

mechanically be pulled through artificial nanopores. Specifically, we have been able to record numerous DNA propagation events through the nanopores, and have been able to implement two of the proposed experiments (the mechano-electrical tug-of-war and DNA relaxation experiments, respectively).

For example, as shown in Fig. 1, we were able to connect a long piece of DNA to a polystyrene bead at one end and then electrophoretically thread the free end of the DNA through a nanopore (applied external potential $=100 \mathrm{mV}$ ). Thus, we created the initial configuration shown in Fig. 1, case A. Then, the optical trap was moved away from the pore membrane at a rate of 6 microns per second (Fig. 1, case B). The force on the bead (the black curve) increased as the DNA was stretched out, and the current through the pore also increased (red curve). Towards the end of the experiment, the force was still increasing, whereas the current had begun to drop, perhaps by bunching of the polymer at the other side of the membrane, near the pore (Fig. 1, case C). Finally, the force increased beyond the maximum optical trapping force, the bead popped out of the trap and relaxed back towards the pore (Fig. 1, case D).

We have now begun to quantify the electrical force vs. the optical force on the DNA, how different retraction rates affect the polymer propagation rate, the influence of different ionic strengths on the force, and the fluctuations of the current and the force. To date, all these measurements $(\mathrm{N}>100)$ are fully consistent with the successful mechanical stretching of single DNA molecules through nanopores.

In conclusion, we have obtained results that indicate that 1) the proposed experiments can be performed, 2) that the numerous bead stickiness, membrane wetting, and flow-chamber problems have been overcome, and 3) that the resolution of the force, current, and position data-streams are sufficiently high to perform the proposed experiments. 
Development of techniques for the study of large macromolecular complexes using X-ray crystallography

\section{Principle Investigators: Robert Tjian}

\section{Project Description}

As our understanding of biological mechanisms becomes more sophisticated, it has become evident that such structural studies are necessary to understand dynamically regulated processes such as DNA replication, transcription, RNA processing, and translation - all of which require the coordinated action of very large multi-subunit enzymes and protein complexes. The purpose of this research is to implement novel strategies for crystallographic analysis of large macromolecular assemblies.

To date, it has been extremely difficult to over-express and assemble large multisubunit complexes in quantities sufficient for crystallographic analysis, consequently this work will largely depend on endogenous complexes isolated directly from cells. First, we will be initiating the large scale immunopurification of human transcription complexes from HeLa cells. Second, we will be biochemically characterizing the complexes to obtain information on the heterogeneity and subunit organization of these large macromolecular assemblies. Purification protocols will be modified based on our biochemical studies to obtain samples that are both structurally and functionally homogenous. Finally, we will be using a combination of robotic and microfluidic devices to crystallographically screen nanoliter amounts of our endogenously purified protein complexes. Once potential crystallization conditions are obtained we will scale up the crystallization conditions and assess the quality of the crystals using the ALS synchrotron.

\section{Accomplishments}

Over the past year we have been able to increase the yield of the immunopurified TFIID complex six fold compared to previous amounts. We have screened various salts and detergents to obtain conditions where we could concentrate TFIID to over $1 \mathrm{mg} / \mathrm{ml}$. Over 1000 different conditions were screened using a nanoliter dispensing robot to obtain solubility profiles of TFIID in various salts and $\mathrm{pH}$ conditions. We will use these solubility profiles to define conditions to further increase the concentration of our TFIID above $1 \mathrm{mg} / \mathrm{ml}$.

Using antibodies against TAF4b, a tissue specific subunit of TFIID, we have been able to purify a TFIID related complex(TFIID-4b) from human Daudi cells(W.L. Liu, K. Geles, and R. Tjian unpublished). We have initiated mass spectrometry experiments to identify the unique components of this tissue specific TFIID related complex. We hope to compare the subunit composition of the TFIID and TFIID-4b complexes to help ascertain any differences related to cell-specific regulation of TFIID function.

Biochemical assays have indicated several unique structural transitions within TFIID. Using chemical crosslinking assays, we have been able to measure conformational changes within TFIID in the presence of TFIIA, activators(VP16, Sp1), and TFIIB In addition, we have preliminary chemical crosslinking data indicating that small molecules, such as ATP and Acetyl-CoA induce structural changes within TFIID. 


\section{Physics Division}

Advanced Monolithic Si Pixel Sensors

for the International Linear Collider

Principal Investigator(s): Marco Battaglia

Project Description: The R\&D program addresses the development of Si pixel sensors of new generation for the detection of ionising particles at the next generation lepton collider, the International Linear Collider (ILC). Physics requirements at the ILC push the vertex tracker specifications to new levels. Monolithic pixel detectors, integrating readout and data processing capabilities into the same Si substrate used for the particle detection offer unprecedented performances in terms of position resolution and readout speed with a minimal amount of material. The recent decision of adopting the superconducting technology for the ILC accelerating cavities allows to better define the detector requirements and further stresses the importance of high pixellisation and fast readout capabilities to minimise the effects of accelerator-induced backgrounds in the physics event reconstruction.

Accomplishments: The first year of the project has been devoted in part to the setup of a facility for detailed characterisation of Si pixel detectors. This allows today to study charge production and collection in pixel detectors and signal cluster shapes by using both radioactive sources and highly focused, low intensity, laser beams. A first detector chip has been designed. It consists of three arrays of pixels of different sizes (from $40 \times 40 \mu \mathrm{m}^{2}$ down to $10 \times 10 \mu \mathrm{m}^{2}$ ). The chip has analog output and most of the data processing is performed off chip on a dedicated readout board. The design has been submitted to the foundry in April and chips have been obtained in July. The chip is realised in a low-noise CMOS process with thick epitaxial layer, which is expected to deliver large signal-to-noise ratio. The chip has been first tested using a $200 \mathrm{keV} e^{-}$beam at NCEM and found to be fully functional. Operating the section with the $10 \times 10 \mu \mathrm{m}^{2}$ pixels gives images of the electron beam with resolution comparable to that of a photographic film. This is extremely encouraging since it proves that charge diffusion is small and charged particle can be localized with accuracies of order of $1 \mu \mathrm{m}$ and excellent two-track separation. Detailed characterisation in the lab is ongoing and a beam test using high energy electrons at the ALS is planned. The characterisation of this chip will allow to proceed to the next step in the detector design, which will consist in implementing in-chip functionalities.

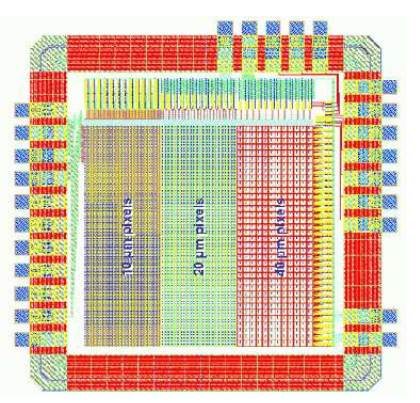




\author{
Designing a Novel Reactor Neutrino Oscillation Experiment \\ for Measuring the Unknown Neutrino Mixing Angle $\theta_{13}$ \\ Principal Investigators: S.J. Freedman, K.M. Heeger, R.W. Kadel, K.-B. Luk
}

\title{
Project Description
}

The purpose of this project is to develop a design concept and research detector technology for a next-generation reactor neutrino oscillation experiment. Using multiple detectors placed at different distances from a nuclear power plant a reactor antineutrino experiment can search for oscillation effects in the $\nu_{\mathrm{e}} \rightarrow v_{\mu, \tau}$ channel and make a precision measurement of the unknown neutrino mixing angle $\theta_{13}$. The discovery of subdominant oscillation and non-zero $\theta_{13}$ would have a profound impact in neutrino physics. The size of $\theta_{13}$ is critical for $\mathrm{CP}$ violation searches in the lepton sector and will define research in neutrino oscillation physics for the next decade and beyond. Neutrino oscillation and CP violation in the lepton sector may lead to leptogenesis and ultimately may explain the observed baryon asymmetry in the Universe.

Our primary goals are to select a suitable experimental site, perform design and engineering studies for future neutrino detectors, prototype detector components, and assess possible backgrounds to this precision neutrino measurement through simulation and environmental measurements. Mechanical and civil engineering studies are needed to demonstrate the technical feasibility of the experiment. An international scientific collaboration is formed to conduct this challenging experiment.

\section{Accomplishments}

In the past year, groups in China, Hong Kong, Russia, and the US developed conceptual designs for an underground neutrino experiment at the Daya Bay nuclear power plant in China. This site is located on the Chinese coast near Hong Kong with nearby mountains and provides the possibility for mounting an underground reactor $\theta_{13}$ neutrino experiment with horizontal access tunnels. At LBNL we performed mechanical engineering work on the detector design as well as conceptual engineering studies on the layout for the underground laboratory. Geologists from LBNL Earth Sciences were involved in the geological site investigation on-site in China working together with colleagues from China and an external tunneling consultant from Fermi National Accelerator Laboratory. Scientific staff at LBNL performed detector simulations in support of engineering studies to characterize the physics potential and the ultimate sensitivity of the experiment. A project summary document was prepared by the US collaboration of the Daya Bay experiment and submitted to the Neutrino Science Assessment Group (NuSAG) for evaluation. Over the past three years scientists from Berkeley have played a lead role in establishing the case for a future reactor neutrino project in the international scientific community. In early 2004 Berkeley Lab formed a R\&D collaboration with the Institute of High Energy Physics (IHEP) in Beijing for the development of this experiment. In November 2005 the cooperation between LBNL and IHEP was renewed at the annual US-China meeting. 


\section{New Directions for Theoretical Physics at the TeV-Scale Principal Investigator: Hitoshi Murayama}

\section{Project Description \\ Particle physicists have known since the time of Fermi (1933) that the TeV scale is an important energy scale in physics. Together with the energy scale of gravity, derived from Newton's constant, it is now viewed as one of the two fundamental energy scales of nature. Much of the development in particle physics has revolved around how we understand these two energy scales. At this time the theoretical understanding of the $\mathrm{TeV}$ scale is extremely poor. The only phenomenologically viable model at hand is the toy model called the Minimal Standard Model, which is unsatisfactory because of its many theoretical shortcomings. Many speculations have been discussed; including such ideas as supersymmetry, large extra dimensions, warped extra dimension(s) and new strong interactions. While all of these ideas have strong theoretical appeal, and some have interesting connections to string theory, arguably the theory of everything, it is fair to say that none of them are free from problems, and their consequences have not been worked out in complete detail. Our goal then is to come up with a consistent theoretical framework that will be confronted with the anticipated beautiful data at the $\mathrm{TeV}$ scale from the Tevatron and the $\mathrm{LHC}$}

\section{Accomplishments}

Our goal can only be approached in a multi-faceted fashion, which brings together somewhat disparate yet connected subfields. To accomplish this, specific problems were addressed by different postdocs and students:

Eric Gimon (with P. Horava) has shown in a five dimensional model universe that string theory can remove the singularities of classical black holes. In subsequent work they have argued that this result could apply in nature and that evidence suggesting black holes violating the Kerr bound on angular momentum, either from Active Galactic Nuclei or solar mass black holes, would constitute indirect evidence in support of string theory.

I. Mitra (with Ratabole and Sharatchandra) has shown that by choosing a nonlocal gauge it is possible to remove infrared divergences from massless Quantum Electrodynamics in dimension $2<\mathrm{d}<4$, thus exhibiting a nontrivial conformal invariant field theory for those space-time dimensions.

V. Hubeny and M. Rangamani (with A. Maloney) have found that two-charge supersymmetric black holes are cured of pathological singularities by string theory corrections and that the resulting gravitational force on an inertial observer is no longer purely attractive. 


\title{
Cross-Divisional
}

LB05001

\author{
Studies of the Health Effects of Nanostructured Materials \\ P.I.s: A.P. Alivisatos, C. Bertozzi, F. Chen, J. Gray, T. McKone \\ Participants: M. Apte, X. Chen, M. Lunden, R. Maddalena, A. Zettl
}

\section{Project Description}

This project was designed to explore the possibility that LBNL could develop a signifcant multidisciplinary initiative to explore the possible adverse health effects of nanoscale materials. Three areas of research were to be pursued to begin assessing such effects. The first involved the study of the sources, environmental fate, and exposure by developing and validating sensors and predictive tools to characterize the behavior of nano-materials in both controlled and ambient environments. The second involved the development of an understanding of the health effects of nanomaterials through studies of the effects of these materials on gene expression, oxidative damage, and signal transduction pathways. The third is the targeted synthesis of specific nanostructured materials for advancing the studies in the first two areas.

\section{Accomplishments}

Addressing the first area, the McKone group and others completed a prospective assessment on the nature of human exposure to nanomaterials. Based on a repository of knowledge accumulated on hundreds of environmental substances, they identified intrinsic chemical properties that are indicative of hazard such as persistence, bioaccumulation and long-range transport. The approach uses experience with "conventional" organic and inorganic pollutants to assess plausible environmental fate and exposure outcomes of several archetypal classes of nano-materials. Efforts were also initiated to adapt and employ particle measurement instrumentation to measure airborne nanomaterial concentrations at the point of release and in the local environment near release. A review was conducted on nanoaerosol dosimetry for formation, measurement, transport and surface deposition, lung deposition, and dosimetry.

In the second area, the Gray and Chen group characterized the molecular and cellular effects of carbon nanomaterials using whole genome microarray and high content imaging approaches. They performed whole genome expression array analysis and high content image analysis-based phenotypic measurements on human skin fibroblast cell populations exposed to multi-wall carbon nano-onions (MWCNOs) and multi-wall carbon nanotubes (MWCNTs). There is evidence that exposing cells to MWCNOs and MWCNTs at cytotoxic doses induces cell cycle arrest and increases apoptosis/necrosis. MWCNTs induce genes indicative of a strong immune and inflammatory response within skin fibroblasts, while MWCNO changes are concentrated in genes induced in response to external stimuli. Promoter analysis of the microarray results demonstrate critical pathway components in the induced signal transduction contributing to the more adverse effects observed upon exposure to MWCNTs as compared to MWCNOs with the Alivisatos group investigated the cytotoxicity of colloidal semiconductor nanocrystal quantum dots. Specifically, they prepared silica coated CdSe quantum dots and examined changes in gene expression that result upon exposure. While some changes were noted, the effects seemed to be similar to those obtained by exposure to simple silica.

The Bertozzi group investigated the cytotoxic effects of carbon nanotubes (CNTs) on cells and new coating methods that abrogate their toxicity and render them biocompatible. They established cell-based assays of CNT toxicity and characterized their effects on mammalian cell lines derived from numerous tissue origins. The CNTs were found to inhibit cell proliferation for all cell lines tested. They developed a carbohydrate-based polymer that mimics the structure of biological mucin glycoproteins, the natural coating found on the surface of living cells. The polymers were assembled on the CNT surfaces and the coated CNTs were evaluated for their physical properties and biocompatibility. Importantly, the coated CNTs showed no toxicity to cultured mammalian cells. The results offer a means to reduce the potential harmful effects of CNTs in biological systems. 
Microscopic Theory of Protein Surface

Principal Investigator: Phillip Geissler

\section{Project Description}

This project aims to develop a quantitative understanding of mechanics and fluctuations at the molecular surfaces of proteins. Of particular interest is the way such surfaces have been designed through evolution to facilitate molecular recognition and binding and to control association into larger assemblies. We will develop and analyze models at several levels of detail to reveal the statistical mechanics of conformational changes on the protein exterior. Results of this analysis will aid in systematic redesign of a protein's composition to modify its interactions with ligands, other proteins, and solvent.

We will examine the degree to which steric constraints on the atomic scale couple motions near the surface to larger reorganization within a molecule's interior. For this purpose we will explore possible repackings of amino acid side chains near a protein's native state as a measure of flexibility and correlations among local rearrangements. With more detailed energetic models we will investigate examples of small molecule binding events that generate large-scale structural changes, such as in certain transmembrane ion channels. In this case we will focus on the transmission of stress from the binding pocket to distant regions of molecules and complexes. Finally, using schematic models, we will study the relationship between spatial distributions of structural fluctuations and the evolution of protein composition.

\section{Accomplishments}

In previous years we invested significant effort in developing computational methods for efficiently sampling proteins' possible side-chain packings. This investment paid off during the last year with applications to a specific molecule, photoactive yellow protein (PYP). We first quantified the diversity of non-native packings permitted by steric constraints alone. The entropy associated with this variety, much larger than that of a well-defined molecular structure but much smaller than for unhindered rotations, raises several interesting questions: Which omitted microscopic details are essential for reducing this side-chain entropy to a physically reasonable value? How are steric correlations among side-chain rotations arranged in space? Do these correlations define collective rearrangements that might be induced by interactions with other molecules? We are addressing these questions in several different ways. (1) From the naturally occurring frequencies of each residue's rotameric states in catalogued protein structures, we have inferred intrinsic rotamer preferences due to covalent bonding and other intraresidue interactions. The entropy of side-chain repackings is roughly halved by these preferences but remains much larger than expected from crystallography and NMR. (2) By artificially restricting the side-chain mobility of one or more residues in and allowing others to fluctuate, we have begun to map out rotamer correlations in space, which can extend over remarkably long distances. We will compare these correlations to those inferred from ultrafast X-ray experiments probing structural rearrangements in PYP. (3) Finally, we have initiated a collaboration with Elizabeth Komives's lab to investigate steric contributions to allostery in thrombin/thrombomodulin complexes. 


\title{
Development and Application of Quantum Monte Carlo (QMC) Methods to Biological Systems
}

\author{
Principal Investigators: William A. Lester, Jr., Graham Fleming, Michael Frenklach
}

\section{Project Description}

The purpose of this research is develop algorithmic improvements to facilitate the calculation of electronic excitations in biological and other large molecular systems using the quantum Monte Carlo (QMC) method. The highest priority is the application of these theoretical and computational developments to the study of singlet-excited states and their role in energy transfer in photosynthetic systems. Significant progress has been made in reducing the computational effort with system size without loss of accuracy that makes possible the promise of application of the highly accurate QMC method to molecules containing more than 300 electrons being studied in this project. At present there is no other first principles quantum chemical method that can be applied to systems as large as those being studied in this project.

\section{Accomplishments}

Over the past year considerable progress has been made in a number of facets of the proposed effort. The areas of advance were software modifications to the present computer code Zori needed to further reduce the computational effort of the demanding QMC algorithm. In addition, efforts have also focused on development of a method to enable the quantitative comparison of QMC-determined bonding distributions with those from obtained using the popular density functional theory (DFT) and other methods. Because of the essential differences between QMC and The detailed examination and testing of new approaches to improve the accuracy of the Monte Carlo random walk leading to better energy convergence are in progress. Further, initial steps have been undertaken in the development of an approach to treat the present systems when embedded in media, such as water and other solvents, that would not significantly impact the accuracy of the present approach. The latter effort has been made possible by the addition of a new postdoctoral associate to the project.

This project has benefited significantly from other Divisions of LBNL. Preliminary calculations that established the feasibility of the present approach were greatly added by images, both static and dynamic, created by the LBNL Visualization Group. In addition, the optimization step that is critical for the quality of the random walks was greatly aided by the involvement of members of the LBNL Computational Research Division. Finally, NERSC staff played an indispensable role in optimal recoding of parts of the QMC code to take full advantage of computer systems and architectures. 
Development of Key Physics and Technical Approaches for the Production of Intense Rare Isotope Beams

Principal Investigator: Jay Marx

Project description

This project utilizes LBNL's core competencies in accelerator science to contribute to providing the advanced capabilities in a number of key areas needed for the next generation of intense rare isotope beams: generation of highly charged ions in ECR sources; transport of intense low energy beams from an ECR ion source to the radio frequency quadrupole (RFQ); codes for end-to-end modeling of intense beams in linacs; superconducting magnet design to meet aperture and field requirements in high radiation environments; and low-level rf control systems to deal with the need to compensate for cavity vibrations and minimize the excess power requirements.

Accomplishments

In the area of simulation and modeling, we have developed an RFQ module inside the parallel particle beam dynamics tracking code, IMPACT. Multiple charge-state particles can be transported through the RFQ as required for RIA and particle loss is checked with a varying aperture size at each location. This module, together with the module of ion beam formation and transport and the rf linac module, enables the IMPACT code to do a full start-to-end simulation for a proposed linac such as the RIA driver linac.

In the area of RFQ accelerators, beam dynamics simulations were successfully done for the SNS RFQ design to benchmark this code for application to the design of an RFQ for RIA and we have succeeded in detailed modeling of the as-built SNS RFQ. This simulation capability was applied to other RFQ structure designs including those that could be considered for RIA.

For the RIA front-end development, the capabilities of existing codes were enhanced to simulate the beam transport from the ECR ion source through the ion beam analyzing section to facilitate endto-end simulations starting from the ECR ion source through the driver linac. The emittance of the existing VENUS ion source was measured over a wide range of extraction fields to understand the suitability of the VENUS design as a basis for an injector for RIA.

The work on low-level rf resulted in a successful test of an rf controller to control microphonics, thus reducing the bandwidth requirements for the high power rf system and the associated costs. Conceptual studies of the layouts of both the frequency distribution and digital rf client systems were completed.

In the magnet development area, a comparison of linac transport systems based on compact quadrupoles with $70 \mathrm{~mm}$ bore and $100 \mathrm{~T} / \mathrm{m}$ gradient vs. high field (up to $15 \mathrm{~T}$ ), smaller bore (30 $\mathrm{mm}$ ) solenoids showed that, although both options are feasible, the compact quadrupole option has significant cost advantages compared with small bore solenoids. For the fragment separator, the baseline super ferric design was compared to current-dominated, accelerator-type designs. It was found that the coil peak field could be increased by a factor of 2-3, with a corresponding increase of the gradient and a reduction of the magnet length. 


\section{Publications List}

AFRD-Lidia LB04019 Critical Accelerator Technologies for Advanced Light Sources

A. Zholents; "Method of an enhanced self-amplified spontaneous emission for x-ray free electron lasers," Physical Review Special Topics - Accelerators and Beams 8: 040701 (12 April 2005).

A. Zholents; "Laser assisted electron beam conditioning for free electron lasers," Physical Review Special Topics - Accelerators and Beams 8: 050701 (17 May 2005).

A. Zholents and G. Penn" "Obtaining attosecond x-ray pulses using a self-amplified spontaneous emission free electron laser," Physical Review Special Topics - Accelerators and Beams 8: 050704 (24 May 2005).

R. Wilcox, J. Staples, \& R. Holzwarth; "Optical synchronization system for femtosecond xray sources," Proceedings of the 2005 Particle Accelerator Conference, Knoxville, Tennessee, May 2005.

J. Qiang, S. Lidia, R. Ryne, \& C. Limborg; "A 3d parallel beam dynamics code for modeling high brightness beams in photoinjectors," Proceedings of the 2005 Particle Accelerator Conference, Knoxville, Tennessee, May 2005.

E. Pedersoli, F. Banfi, B. Ressel, S. Pagliara, C. Giannetti, G. Galimberti, S. Lidia, J. Corlett, G. Ferrini, \& F. Parmigiani; "Evidence of vectorial photoelectric effect on Copper," Applied Physics Letters 87: 081112 (17 August 2005).

J. Staples and R. Wilcox; "Fiber transmission stabilization by optical heterodyning techniques and synchronization of mode-locked lasers using two spectral lines," Proceedings of the $27^{\text {th }}$ International Free Electron Laser Conference, Stanford, California, August 2005.

F. Banfi, G. Ferrini, E. Pedersoli, S. Pagliara, C. Giannetti, G. Galimberti, S. Lidia, J. Corlett, B. Ressel, \&F. Parmigiani; "Monte carlo transverse emittance study on Cs2Te," Proceedings of the $27^{\text {th }}$ International Free Electron Laser Conference, Stanford, California, August 2005.

A. Zholents; "Attosecond x-ray pulses from free-electron lasers," Laser Physics 15(6): 855862 (September 2005).

J. Qiang, S. Lidia, R. Ryne, \& C. Limborg-Deprey; "A three-dimensional quasi-static model for high brightness beam dynamics simulation," submitted for publication to Physical Review Special Topics - Accelerators and Beams.

\section{AFRD-Logan LB05022 Enabling High Energy Density Physics at LBNL}

\section{Refereed Publications}

W. P. Leemans, E. Esarey, J. van Tilborg, P. A. Michel, C. B. Schroeder, Cs. T'oth, C. G. R. Geddes, and B. A. Shadwick: "Radiation from laser accelerated electron bunches: coherent terahertz and femtosecond x-rays," IEEE Trans. Plasma Sci. 33 (1), 8--22 (February 2005); LBNL-56584.

C.B. Schroeder, E. Esarey, and W.P. Leemans; "Beam conditioning by Thomson scattering," Phys. Rev. Lett. 93(19): 194801 (November 2004); LBNL-54095 [also published in Virtual Journal of Ultrafast Science, December 2004]. 


\section{Conference Proceedings}

B.A. Shadwick, E. Esarey, W.P. Leemans; "Beam dynamics in plasma-based accelerators including the effects of betatron radiation", P. Michel, C.B. Schroeder, Bull. American Phys. Society DPP 2005 vol. 50(8): 185 (2005).

A. Friedman, J. J. Barnard, R. J. Briggs, et al; "Highly Compressed Ion Beams for High Energy Density Science," in Proceedings of the Particle Accelerator Conference 2005, May 16-20, Knoxville, TN.

J. J. Barnard, R. J. Briggs, D. A. Callahan, et al; "Accelerator and Ion Beam Tradeoffs for Studies of Warm Dense Matter," in Proceedings of the Particle Accelerator Conference 2005, May 16-20, Knoxville, TN.

R. C. Davidson, B. G. Logan, J. J. Barnard, et al; "US Heavy Ion Beam Research for Energy Density Physics Applications and Fusion," Fourth International Conference on Inertial Fusion Sciences and Applications (IFSA05), 4 - 9 September 2005, Biarritz, France. Princeton Plasma Physics Report PPPL-4100.

J.J. Barnard (Ed.), R.O. Bangerter, R.J. Briggs, et al; Proceedings of the Workshop on Accelerator Driven High Energy Density Physics, October 26-29, Berkeley, CA; LBNL57518.

\section{Invited Talks}

W.P. Leemans, "Laser Plasma Sources for High Brightness Beams: from THz to X-rays", Free Electron Laser Conference 2005, Stanford, CA, August 2005.

W.P. Leemans, "THz and X-rays from high brightness laser accelerated electron beams", $10^{\text {th }}$ ICOMP meeting, Orford, Quebec, Canada, October 2005.

C. B. Schroeder, "Electrons, x-rays, and THz radiation from laser-plasma accelerators", presented at the US-Japan Workshop on Ultra-Intense Laser Plasma Interactions, Austin, TX, October 2005.

C. Tóth, W.P. Leemans, C.G.R. Geddes, J. van Tilborg, P. Michel, C. Filip, C. Schroeder, and E. Esarey: Diagnostics of Plasmas and Electron Beams in Laser Wake-Field Accelerator Research, 12th International Symposium on Laser-Aided Plasma Diagnostics, LAPD-12, Snowbird, UT, September 26-29, 2005 (Invited) Paper VIIB-01.

A. Friedman, "Highly Compressed Ion Beams for High Energy Density Science", 2005 Particle Accelerator Conference.

J, Wurtele, "Physics, Technology and Applications of Advanced Accelerators", U. Texas Physics Department Colloquium, May 2005.

R. Davidson, "US Heavy Ion Beam Research for Energy Density Physics Applications and Fusion," Presented at the Fourth International Conference on Inertial Fusion Sciences and Applications (IFSA05).

AFRD-Ryne LB03021 Optimal Solvers for Infinite-Dimensional Hamiltonian Systems

B. Terzic and I.V. Pogorelov, "Wavelet-Based Poisson Solver for Use in Particle-in-Cell Simulations", Ann. N.Y. Acad. Sci., 1045: 55-67 (2005).

B. Terzic, D. Mihalcea, C.L. Bohn, and I.V. Pogorelov, "Wavelet-Based Poisson Solver for Particle-in-Cell Simulations", in Proc. 2005 Part. Accel. Conf., 2601 (2005); LBNL57627.

I.V. Pogorelov, J.Qiang, R.D. Ryne, and R.L.Gluckstern, "Symplectic Discretization Schemes for Infinite-Dimensional Hamiltonian Systems," in preparation. 
I.V. Pogorelov and B. Terzic, "Compression, Denoising, and Convergence Acceleration in Wavelet-Based Poisson Solvers for Particle-in-Cell Simulations", in preparation.

AFRD-Vay/Furman LB03028 Electron Production and Collective Field Generation in Intense $\underline{\text { Particle Beams }}$

R. H. Cohen; A. Friedman, M. Kireeff Covo, S. M. Lund, A. W. Molvik, F. M. Bieniosek, P. A. Seidl, J.-L. Vay, P. Stoltz, S. Veitzer; "Simulating Electron Clouds in Heavy-Ion Accelerators," Phys. Plasmas, 12(5): 56718-1-10 (2005).

R. H. Cohen; A. Friedman, S. M. Lund, A. W. Molvik, A.W.; Azevedo, J.-L. Vay, P. Stoltz, S. Veitzer; "Simulating Electron Cloud Effects in Heavy-Ion Beams," Nuclear Inst. and Methods in Physics Research A 544: 210-215, (2005).

R. H. Cohen; A. Friedman, S. M. Lund, A. W. Molvik, E. P. Lee, A. W.; Azevedo, J.-L. Vay, P. Stoltz, S. Veitzer; "Electron-Cloud Simulation and Theory for High-Current HeavyIon Beams," Phys. Rev. Spec. Top.-AcBeams 7: 124201 (2004).

M. Kireeff Covo, J.J. Barnard, R.H. Cohen, et al; "Beam Energy Scaling of Ion-Induced Electron Yield from K+ Ions Impact on Stainless Steel Surfaces," Particle Accelerator Conference, Knoxville, TN, May 16-20, 2005; url: http://accelconf.web.cern.ch/accelconf/p05/PAPERS/FPAP033.PDF.

A. W. Molvik, P. A. Seidl, F. M. Bieniosek, R. H. Cohen, M. Kireeff Covo, L. Prost; "Experimental Studies of Electrons in a Heavy-Ion Beam," Nuclear Inst. and Methods in Physics Research A 544: 194-201, (2005).

A. W. Molvik, M. Kireeff Covo, R. H. Cohen, A. Friedman, J. J. Barnard, J.-L. Vay, P. A. Seidl, J. L. Vujic; "Experiments Studying Desorbed Gas and Electron Clouds in Ion Accelerators," Invited Paper, Particle Accelerator Conference, Knoxville, TN, May 1620, 2005.

P. H. Stoltz, S. A. Veitzer, R. H. Cohen, A. W. Molvik, J.-L. Vay, "Energy loss, range, and electron yield comparisons of the CRANGE ion-material interaction code," Nuclear Inst. and Methods in Physics Research A 544: $502-505$ (2005).

P. H. Stoltz, S. Veitzer, R. H. Cohen, A. W. Molvik, J.-L. Vay; "Simulation of heavy ion induced electron yield at grazing incidence," Physical Review Special TopicsAccelerators and Beams, 7(10) (2004).

J.-L. Vay, M. A. Furman, P. A. Seidl, R. H. Cohen, A. Friedman, D. P. Grote, M. Kireeff Covo, A. W. Molvik; "Filling in the Roadmap for self-Consistent Electron Cloud and Gas Modeling," Invited Paper, Particle Accelerator Conference, Knoxville, TN, May 16-20, 2005; url: http://accelconf.web.cern.ch/accelconf/p05/PAPERS/ROPB006.PDF.

J.-L.Vay, M. A. Furman, R. H. Cohen, A. Friedman, D. P. Grote; "Initial self-consistent 3D Electron Cloud Simulations of the LHC Beam with the code WARP+POSINST," Particle Accelerator Conference, Knoxville, TN, May 16-20, 2005; url: http://accelconf.web.cern.ch/accelconf/p05/PAPERS/FPAP016.PDF.

S.A. Veitzer, P. Stoltz, R.H. Cohen, A.W. Molvik, J.-L. Vay; “A Cross-Platform Numerical Model of Ion-Wall Collisions,” Accelerator Conference, Knoxville, TN, May 16-20, 2005; url: http://accelconf.web.cern.ch/accelconf/p05/PAPERS/FPAP021.PDF.

\section{AFRD-Zolotorev LB05039 Low Energy Spread Electron Source}

M. Zolotorev, E. Commins, P. Denes, Z. Hussain, G. Lebedev, S. M. Lidia, D. Robin, F. Sannibale, R. W. Schoenlein, R. Vogel, W. Wan, S. Heifets; "An ultra-bright pulsed electron beam with low longitudinal emittance," Proceedings of the 2005 Particle 
Accelerator Conference, Knoxville, Tennessee, p. 770, May 2005; url:

http://www.sns.gov/pac05/.

M. Zolotorev; "An Ultra-Bright Pulsed Electron Beam with Low Longitudinal Emittance," Workshop on The Physics and Applications of High Brightness Electron Beam, Erice, Italy, October 05; invited talk, url: http://www.physics.ucla.edu/PAHBEB2005/program/index.htm.

During FY05 we also filed an Invention Disclosure covering the source concept.

ALS-Howells LB04013 Coherent X-ray Diffraction Imaging (CXDI)

S. P. Hau-Riege, H. Szoke, H. N. Chapman, A. Szoke, S. Marchesini, A. Noy, H. He, M. Howells, U. Weierstall, J. C. H. Spence; "SPEDEN: Reconstructing single particles from their diffraction patterns," Acta Crys A, A60, 294-305 (2004),

T. Beetz, M. R. Howells, C. Jacobsen, C.-C. Kao, J. Kirz, E. Lima, T. O. Mentes, H. Miao a, C. Sanchez-Hanke, D. Sayre, D. Shapiro; "Apparatus for X-ray diffraction microscopy and tomography of cryo specimens," Nucl. Instrum. Meth. A, 545, 459-468 (2005).

M. R. Howells, T. Beetz, H. N. Chapman, C. Cui, J. M. Holton, C. J. Jacobsen, J. Kirz, E. Lima, S. Marchesini, H. Miao, D. Sayre, D. A. Shapiro, J. C. H. Spence; "An assessment of the resolution limitation due to radiation-damage in x-ray diffraction microscopy," Accepted by Journal of Electron Spectroscopy and Related Phenomena, (2005).

D. Shapiro, P. Thibault, T. Beetz, V. Elser, M. Howells, C. Jacobsen, J. Kirz, E. Lima, H. Miao, A. Neiman, D. Sayre; "Biological Imaging by Soft X-ray Diffraction Microscopy," Proceedings of the National Academy of Sciences of the USA, 102, 15343-15346 (2005).

H. N. Chapman, A. Barty, S. Marchesini, A. Noy, S. H.-. Riege, C. Cui, M. R. Howells, R. Rosen, H. He, J. C. H. Spence, U. Weierstall, T. Beetz, C. Jacobsen, D. Shapiro; "Highresolution ab initio Three-dimensional X-ray Diffraction Microscopy," Accepted by Journal of the Optical Society of America A, (2005),

ALS-Kirz LB04044 Lensless Imaging of Yeast Cells

D. Shapiro, P. Thibault, T. Beetz, V. Elser, M. Howells, C. Jacobsen, J. Kirz, E. Lima, H. Miao, A. M. Neiman, and D. Sayre; "Biological imaging by soft x-ray diffraction microscopy," Proc. Nat. Acad. Sci. 102, 15343-15346 October 25, 2005.

E. Lima, D. Shapiro, P. Thibault, T. Beetz, V. Elser, M. Howells, X. Huang, C. Jacobsen, J. Kirz, H. Miao, A. M. Neiman, A. Stewart, and D. Sayre; "Diffraction Microscopy: Reconstruction of the complex-valued image of a yeast cell," Proc. X-Ray Microscopy 2005 (in press).

ALS-Martin LB05024 Left-handed Nanoscale Meta-Materials: Towards the Optical Domain

M.C. Martin, Z. Hao, A. Liddle, E.H. Anderson, W.J. Padilla, D. Schurig, D.R. Smith; "Fabrication and Optical Measurements of Nanoscale Meta-Materials," in IRMMWTHz2005 Conference Proceedings, IEEE; LBNL-58990.

ALS-Rotenberg LB05033 NanoARPES: A New Detector for Nanometer-scale Electronic Structure Measurements

E. Rotenberg, A. Bostwick; "Space-charge simulations of ARPES with nanometer probe sizes," draft in preparation. 
A. Bostwick, "New Visions in Bandmapping: Zone Plate Approaches to nanoFocus," Xray Microscopy Meeting (XRM), 26-30 July 2005, Egret Himejei Japan.

ALS-Scholl LB04030 Ultra-fast Magnetization Dynamics

F. Bartelt, A. Comin, J. Feng, J. Nasiatka, H. A. Padmore, A. Scholl, and A. T. Young; "Ultrafast magnetization dynamics studies using an x-ray streak camera," in Ultrafast $X$ ray Detectors, High-Speed Imaging, and Applications, edited by Stuart Kleinfelder, Dennis L. Paisley, Zenghu Chang, Jean-Claude Kieffer, Jerome B. Hastings, Proc. SPIE 5920: 592019 (2005).

J.Feng, W.Wana, J.Qiang, A. Bartelt, A. Comin, A. Scholl, J.Byrd, R. Falcone, M.Greaves, G.Huang, A. MacPhee, J.Nasiatka, K. Opachich, D. Weinstein, T. Young and H.A.Padmore; "An ultra-fast x-ray streak camera for the study of magnetization dynamics," in Ultrafast X-ray Detectors, High-Speed Imaging, and Applications," edited by Stuart Kleinfelder, Dennis L. Paisley, Zenghu Chang, Jean-Claude Kieffer, Jerome B. Hastings, Proc. SPIE 5920: 592009 (2005).

CH-Ahmed LB04038 Gas Phase Studies of the Building Blocks of Life

D. S. Peterka and M. Ahmed; "Atoms to aerosols- the chemical dynamics beamline," Synchrotron Radiation News 18 (2005) 35.

K. R. Wilson, M. Jimenez-Cruz, C. Nicolas, L. Belau, S. R. Leone, and M. Ahmed; "Thermal vaporization of biological nanoparticles: Fragment-free VUV photoionization mass spectra of tryptophan, phenylalanine-glycine-glycine and $\beta$-carotene," J. Phys. Chem. A (in press).

K. R. Wilson, L. Belau, M. Jimenez-Cruz, C. Nicolas, S. R. Leone, and M. Ahmed; "Direct determination of the ionization energy of histidine with VUV synchrotron radiation," submitted to Int. J. Mass. Spectrom.

K. R. Wilson, D. S. Peterka, M. Jimenez-Cruz, S. R. Leone, and M. Ahmed; "VUV Photoelectron imaging of biological nanoparticles - Ionization energy determination of nanophase glycine and phenylalanine-glycine-glycine," draft to be submitted to J. Phys. Chem.

C. Nicolas, L. Belau, X. N. Tang, S. R. Leone and M. Ahmed; "Single photon ionization of the DNA base- guanine with synchrotron radiation," draft to be submitted to $J$. Am. Chem. Soc. (in preparation).

L. Belau, K. R. Wilson, S. R. Leone, and M. Ahmed; "VUV photoionization dynamics of solvated histidine and cytosine," draft to be submitted to J. Phys. Chem. A.

CH-Rescigno LB04029 Advanced Computational Methods for Photon-Molecule Collision Processes

W. Vanroose, F. Martín, T, N. Rescigno and C. W. McCurdy; "Nonperturbative Theory of Double Photoionization of the Hydrogen Molecule," Phys. Rev. A 70, 050703 (2004).

F. Martín, D. A. Horner, W. Vanroose, T. N. Rescigno and C. W. McCurdy; "First Principles Calculations of the Double Photoionization of Atoms and Molecules using B-splines and Exterior Complex Scaling," Proceedings of the Thirteenth International Symposium on Polarization and Correlation in Electronic and Atomic Collisions and the International Symposium on (e,2e), Double Photoionization and Related Topics, Buenos Aires, 2005 (to be published). 
T. N. Rescigno, D. A. Horner, F. L. Yip and C. W. McCurdy; “A Hybrid Approach to Molecular Continuum Processes Combining Gaussian Basis Functions and the Discrete Variable Representation," Phys. Rev. A 72, 022705 (2005).

W. Vanroose, F. Martín, T. N. Rescigno and C. W. McCurdy; "Complete Photo-Induced Breakup of the $\mathrm{H}_{2}$ Molecule as a Probe of Molecular Electron Correlation," Science (accepted for publication).

CH-Tilley LB05037 New Approach for the Catalysis Conversion of Methane and Other Inert Hydrocarbons

J. Gavenonis and T. D. Tilley; "Synthesis and reactivity of bis(heptamethylindenyl)-yttrium $\left(\right.$ Ind $\left.{ }_{2} \mathrm{Y}\right)$ complexes containing alkyl and hydride ligands: crystal structure of Ind ${ }_{2}$ YCl(THF)," Journal of Organometalic Chemistry 689:870 (2004).

F.-G. Fontaine and T. D. Tilley; "Control of selectivity in the hydromethylation of olefins via ligand modification in scandocene catalysts," Organometallics 24:4340 (2005).

CS-Chevassut LB05008 Cryptographic Foundations for New Generation Distributed Systems

M. Abdalla, O. Chevassut, P. A. Fouque, D. Pointcheval, A Simple; "Threshold Authenticated Key Exchange from Short Exponents," Proceedings of the Asiacrypt Conference, December 2005.

E. Bresson, O. Chevassut, D. Pointcheval; "A Security Solution for IEEE 802.11's Ad hoc Mode: Password-Authentication and Group Diffie-Hellman Key Exchange," International Journal of Wireless and Mobile Computing - Special Issue on Security of Computer Network and Mobile Systems, 2006 (in press).

M. Abdalla, O. Chevassut, D. Pointcheval; "One-time Verifier-based Encrypted Key Exchange," Proceedings of the International Workshop on Practice and Theory in Public Key Cryptography, February 23-26, 2005.

E. Bresson, O. Chevassut, D. Pointcheval; "Mutual Authentication and Group Key Agreement for Low-Power Mobile Devices," Journal of Computer Communications Special Issue on Security and Performance in Wireless and Mobile Networks, Elsevier, 2004.

L. Fang, S. Meder, O. Chevassut; "Secure Password-based Authenticated Key Exchange for Web Services," ACM Workshop on Secure Web Services, November 2004.

M. Abdalla, E. Bresson, O. Chevassut, D. Pointcheval; "Provably-Secure Password-based Authentication in TLS," draft in preparation.

Chevassut, P. A. Fouque, P. Gaudry, D. Pointcheval; "Key Derivation and Randomness Extraction," ACM Conference on Computer and Communications Security, draft in preparation.

CS-Hamann LB03012 Interactive Visualization Methods for Exploration and Comparison of Multi-Billion Base Pair Sequence Data

Shah, N., Teplitsky, M.V., Pennacchio, L.A.,, Hugenholtz, P., Hamann, B. and Dubchak, I.L. (2005); "SNP-VISTA: An interactive SNPs visualization tool, poster presentation," in: Frazer, K.A., Hudson, T.J., Paeaebo, S., and Wilson, R.K., eds., Proceedings of The Biology of Genomes, Cold Spring Harbor Laboratory Press, Woodbury, New York, p. 242 (presented at: "The Biology of Genomes," Cold Spring Harbor, New York, May 2005). 
Shah, N., Teplitsky, M.V., Minovitsky, S., Pennacchio, L.A., Hugenholtz, P., Hamann, B. and Dubchak, I.L.; "SNP-VISTA: An interactive SNPs visualization tool," to appear in Bioinformatics, Oxford University Press; url: http://genome.lbl.gov/vista/snpvista.

Weber, G.H., Luengo Hendriks, C.L., Keraenen, S.V.E., Dillard, S.E., Sudar, J.D. and Hamann, B.; "Visualization tools for three-dimensional gene expression data in Drosophila," poster presentation (374B), in: Aurora, K., Warrior, R. and Laski, F.A., eds., Program and Abstracts Volume of 46th Annual Drosophila Research Conference, The Genetics Society of America, Bethesda, Maryland, p. 213 (presented at: "46th Annual Drosophila Research Conference," San Diego, California, March/April 2005).

Weber, G.H., Luengo Hendriks, C.L., Keraenen, S.V.E., Dillard, S.E., Ju, D.Y., Sudar, J.D. and Hamann, B.; "Visualization for validation and improvement of three-dimensional segmentation algorithms," in: Brodlie, K.W., Duke, D.J. and Joy, K.I., eds., Data Visualization 2005 (Proceedings of "EuroVis 2005"), Eurographics Association, Aire-laVille, Switzerland, pp. 93-100 (presented at: "Joint Eurographics-IEEE VGTC Symposium on Visualization (EuroVis 2005)," Leeds, United Kingdom, June 2005).

Knowles, D.W., Luengo Hendriks, C.L., Keraenen, S.V.E., Fowlkes, C.C., Weber, G.H., Ruebel, O., Peng H., DePace, A., Hamann, B., Sudar, J.D., Eisen, M.B., Biggin, M.D. and Malik J.; "Berkeley Drosophila transcription network project: 3D blastoderm gene expression atlas," presented at: "Genome Informatics," Cold Spring Harbor, New York, October/November 2005.

Crivelli, S.N., Kreylos, O., Hamann, B., Max, N.L. and Bethel, E.W.; "ProteinShop: A tool for interactive protein manipulation and steering," Journal of Computer-Aided Molecular Design (JCAMD) 18, Kluwer Academic Publishers, pp. 271-285.

CS-Hoschek LB05015 Peer-to-Peer Resource Discovery Framework

W. Hoschek; "A Quantitative Comparison of Binary XML Encodings for Grid Services," submitted to IEEE International Parallel \& Distributed Processing Symposium 2006.

Web pages with software and documentation developed as part of the research in this project are http://www.dsd.lbl.gov/firefish/ and http://www.dsd.lbl.gov/nux/.

CS-Meza LB02045 Parallel Methods for Robust Optimization and Uncertainty Quantification

J.C. Meza, R.A. Oliva, P.D. Hough, P.J. Williams; "OPT++: An object-oriented nonlinear optimization framework," submitted to ACM Transactions on Mathematical Software.

CS-Oliker LB05029 Enhancing Commodity Processors with Vector Components for Increased Scientific Productivity

S.Williams, J. Shalf, L. Oliker, P. Husbands, S. Kamil, K. Yelick; "The Potential of the CELL Processor for Scientific Computing," submitted to IEEE International Parallel \& Distributed Processing Symposium, April 2006.

J. Gebis, D. Patterson; "Embracing and Extending 20th Century Instruction Set Architecture: A Vector Case Study," submitted to IEEE Computer. 
CS-Pinar LB05031 Advanced Computational Tools for Electric Power Systems

V. Donde, V. Lopez, B. Lesieutre, A. Pinar, C. Yang, and J. Meza; "Identification of Severe Multiple Contingencies in Electric Power Networks," Proceedings of North American Power Symposium, October, 2005 (also available as a technical report, LBNL-57994).

V. Donde, V. Lopez, B. Lesieutre, A. Pinar, C. Yang, and J. Meza; "Identification of Severe Multiple Contingencies in Electric Power Networks,"draft to be submitted to IEEE Transactions on Circuits and Systems.

CS-Romano LB05032 Statistical Feature Modeling for Scientific Data Via Basis Decomposition

Blakely, E.A., Schwarz, R.I., Thompson, A.C., Bjornstad, K.A.. Chang, P.Y., Rosen, C.J., Sudar, D., Romano, R., Parvin, B.; "X-ray Microbeam Bystander Studies With Human Mammary Epithelial Cells and Fibroblasts," DOE Low Dose Radiation Research Program Workshop V, Bethesda, Maryland, April, 2005.

Costes, S. V., Rydberg, B., Ravani, S., Romano, R., Parvin, B., Barcellos-Hoff, M. H.; "Automatic Analysis and Modeling of Phosphorylated H2AX and ATM Foci Formation After Exposure to X-ray and HZE," Bioastronautics Investigators' Workshop, Galveston, TX, January 2005.

CS-Yelick LB03030 Evaluation of Computer Architecture Alternatives

J. Shalf, S. Kamil, L. Oliker, D. Skinner; "Analyzing Ultra-Scale Application Communication Requirements for a Reconfigurable Hybrid Interconnect," Supercomputing 2005, Seattle, WA, November 2005.

S. Kamil, J. Shalf, L. Oliker, D. Skinner; "Understanding Ultra-Scale Application Communication Requirements," IEEE International Symposium on Workload Characterization (IISWC) 2005.

S. Kamil, P. Husbands, L. Oliker, J. Shalf, and K. Yelick; "Impact of Modern Memory Subsystems on Cache Optimizations for Stencil Computations", 3rd Annual ACM SIGPLAN Workshop on Memory Systems Performance (MSP) 2005.

ESD-Alvarez-Cohen LB03008 Real-time PCR With Reverse Transcription for Quantification of Chlorinated Solvent Degradation

Rahm, B.G., Chauhan, S., Holmes, V.F., Macbeth, T W., Sorenson, K. S., and L. AlvarezCohen; "Molecular Characterization of Microbial Populations at Two Sites with Differing Reductive Dechlorination Abilities," Biodegradation, in press.

Freeborn R. A., K. A. West V. K. Bhupathiraju, S. Chauhan, , B. G. Rahm, R. E. Richardson, L. Alvarez-Cohen; "Phylogenetic Analysis of TCE-Dechlorinating Consortia Enriched on a Variety of Electron Donors," Environmental Science \& Technology, 39(21):8358-8368 (2005).

Johnson, D. R., Lee, P. K. H., Holmes, V. F., and L. Alvarez-Cohen; “An Internal Reference Technique for Accurately Quantifying Specific mRNAs by Real-Time PCR with Application to the tceA Reductive Dehalogenase Gene," App. \& Env. Microbiology, in press.

Johnson, D. R., Lee, P. K. H., Holmes, V. F., and L. Alvarez-Cohen; “Transcriptional Expression of the tceA Gene in a Dehalococcoides-Containing Microbial Enrichment," Applied and Environmental Microbiology, 71:3866-3871 (2005). 
ESD-Benson LB05043 Scientific Basis for Advanced Geologic Storage Technologies

Tomutsa, L., D. Silin, S. Benson and T. Patzek; "Synchrotron Microtomography in $\mathrm{CO}_{2}$ Geosequestration Research," poster presentation given at the American Geophysical Union (AGU) Fall Meeting, San Francisco, CA, December 5, 2005.

ESD-Borglin LB04005 Development of Biosensors for Endocrine Disrupting Compounds in Agricultural Watersheds

C. G. Campbell, S.E. Borglin, F.B. Green, A. Grayson, E. Wozei, \& W.T. Stringfellow; "Environmental Monitoring, Fate, and Transport of Estrogenic Endocrine Disrupting Compounds: A Review," submitted to Chemosphere.

C.G. Campbell, S.E. Borglin., W.T. Stringfellow., F.B.Green, and A. Grayson; "Biologically-based sensors for endocrine disrupting compounds in water," conference paper to the American Society of Civil Engineers, Alaska, May 15-20, 2005.

ESD-DePaolo LB05011 Micro-characterization and Chemical Micro-dynamics of Atmospheric Mineral Dust

G. Lu, D. DePaolo, Q. Kang, and D. Zhang; "Lattice Boltzmann Simulation of Isotope Kinetics in Crystal Growth," Eos Trans. AGU, 86(52), Fall Meet. Suppl., Abstract H33A1364 (2005).

J.N.Christensen, S.S. Cliff, R.A. VanCuren, L. Tomutsa, K.D. Perry, D.J. DePaolo; "Using Isotopes to Track the Passage of a Spring 2002 Dust Storm Over Hefei, China," Eos Trans. AGU, 86(52), Fall Meet. Suppl., Abstract H31B-0839.

ESD-Gilbert LB05003 Behavior and Impact of Nanoparticles in the Environment

B. Gilbert and J. F. Banfield; "Molecular-Scale Processes Involving Nanoparticulate Minerals in Biogeochemical Systems," Reviews in Mineralogy and Geochemistry 59, in press (2005).

H. Zhang, B. Chen, B. Gilbert and J. F. Banfield; "Kinetically controlled formation of a novel nanoparticulate $\mathrm{ZnS}$ with mixed cubic and hexagonal stacking," Journal of Materials Chemistry, in press (2005).

Z. Lin, B. Gilbert, Q. Liu, Y. Ren, F. Huang; “A thermodynamically stable nanocrystalline material," submitted to Journal of the American Chemical Society.

B. Gilbert, F. Huang, Z. Lin, C. Goodell, H. Zhang, and J.F. Banfield; "Surface chemistry controls the crystallinity of $\mathrm{ZnS}$ nanoparticles," submitted to Nanoletters.

B. Gilbert, C. Kim and G. Lu; "Partially-reversible formation of aqueous clusters of goethite nanoparticles," draft in preparation to be submitted to Science.

B. Gilbert; "Pair distribution function analysis of the structure of nanoparticles," draft in preparation to be submitted to the Journal of Applied Crystallography.

B. Gilbert, H. Zhang, B. Chen, J. F. Banfield; "The compressibility of ZnS nanoparticles," draft in preparation to be submitted to Physical Review $B$. 
ESD-Miller LB04025 The California Water and Energy Systems: an Approach for Addressing Future Crises

J. Jin, J. and N.L. Miller; "An analysis of climate variability in mountainous regions," in press J. Hydrometeorology, LNBL-53845.

J. Jin and N.L. Miller; "Relationship Between Atmospheric Circulation and Snowpack in the western United States,' Hydrological Processes, in press.

R.M. Maxwell and N.L. Miller; "Development of a coupled land surface and groundwater model for use in watershed management," J. Hydrometeorology, June 2005: $233-247$.

L. Pan, J. Jin, Y-S Wu, and N.L. Miller; "Analysis of soil moisture and boundary layer response using the coupled CLM3-TOUGH2 model in the Valdai Watershed," draft to be submitted to J. Hydrometeorology.

G.W. Su, N.W.T. Quinn, P.J. Cook, and W. Shipp; "Miniaturization of the flowing fluid electrical conductivity logging technique," submitted to Ground Water.

ESD-Moridis LB04009 Next Generation Codes for Modeling Subsurface Processes

Finsterle, S., G. J. Moridis, and M. B. Kowalsky; TOUGH-Fx Developer's Guide: Programming Standard, Module and Interface Development, draft in preparation to be issued as LBNL Report.

Kowalsky, M., S. Finsterle, J. Peterson, S. Hubbard, Y. Rubin, E. Majer, A. Ward, and G. Gee; "Estimation of field-scale soil hydraulic parameters and dielectric parameters through joint inversion of GPR/hydrological data," Water Resour. Res., in press.

Moridis, G. J., M. B. Kowalsky, and K. Pruess; TOUGH-Fx/Hydrate v1.0 user's Manual: A Code for the Simulation of System Behavior in Hydrate-Bearing Geologic Media, in preparation as Report LBNL-58950, Lawrence Berkeley National Laboratory, Berkeley, Calif., 2006.

Moridis, G.J. M. Kowalsky and K. Pruess; "Depressurization-Induced Gas Production From Class 1 Hydrate Deposits," presented at the 2005 SPE Annual Technical Conference and Exhibition, October 9-12, 2005, Dallas, Texas, USA (SPE paper 97266 - LBNL number pending).

Moridis, G.J. and M. Kowalsky; "Gas Production from Unconfined Class 2 Hydrate Accumulations in the Oceanic Subsurface," in Economic Geology of Natural Gas Hydrates, M. Max, A.H. Johnson, W.P. Dillon and T. Collett, Editors, Kluwer Academic/Plenum Publishers, in press; LBNL-57299, 2005.

ESD-Sposito LB04034 Advancing the Next Generation of Rock-Fluid Imaging and Stimulation Technologies

W.-C. Lo, G. Sposito, and E. Majer; "Low-frequency dilatational wave propagation through unsaturated porous media containing two immiscible fluids," presented at the American Geophysical Union Annual Fall Meeting (San Francisco, December 2004).

E. L. Majer, S. Pride, T. Daley, W.-C. Lo, and G. Sposito; "Modeling and field results from seismic stimulation," presented at the $17^{\text {th }}$ International Symposium on Nonlinear Acoustics (Pennsylvania State University, July 2005).

W.-C. Lo, G. Sposito, and E. Majer; "Wave propagation through an elastic porous medium containing two immiscible fluids," Water Resources Research 41, W02025 (20 pp., 2005). 
W.-C. Lo, G. Sposito, and E. Majer; "Low-frequency dilatational wave propagation through unsaturated porous media containing two immiscible fluids," Transport in Porous Media, in press.

W.-C. Lo, G. Sposito, and E. Majer; "Low-frequency dilatational wave propagation through fully-saturated porous media," Advances in Water Resources, in press.

S. R. Pride, E. G. Flekkøy; "Seismic stimulation for enhanced oil recovery," submitted to Journal of Petroleum Science and Engineering.

ESD-Steefel LB05036 Biogeochemical Reaction Rates and Pathways in Porous Media

Yang, L., C.I. Steefel, J. Larsen; "Kinetics of kaolinite precipitation and dissolution under circumneutral pH conditions," presented at AGU Fall Meeting 2005.

Steefel, C.I., D.J. DePaolo, P.C. Lichtner; "Reactive transport modeling: An essential tool and a new research approach for the Earth sciences," Earth and Planetary Science Letters 240: 539-558 (2005).

EG-Denes LB05010 Novel Imaging Detectors for Materials and Biology Research

H. A. Padmore; "Development of high speed CCD-based detectors for synchrotron radiation research," presented at IWORID7 Grenoble, April, 2005.

H. A. Padmore; "Development of high speed CCD-based detectors for synchrotron radiation research," presented at SPIE San Diego, August, 2005.

P. Denes; "High-speed, high-DQE detectors for electron microscopy," presented at Microscopy and Microanalysis 2005: Honolulu, August, 2005.

EETD-Fischer LB05012 Constraining Ammonia Emission Inventories for Control of Air Quality

M.L. Fischer, D. Littlejohn; “Time Resolved Measurement of Ecosystem-Atmosphere $\mathrm{NH}_{3}$ Exchange Using the Eddy Covariance Technique," presented at American Geophysical Union, Fall 2005 (12/9/05). Conference.

M.L. Fischer, D. Littlejohn; "An Instrument for Eddy Covariance Flux Measurement of $\mathrm{NH}_{3}$," draft to be submitted to Journal of Atmospheric and Oceanic Technology.

EETD-Koskecki LB04028 Novel High-temperature Membranes and Electrocatalyst Structures for Fuel Cells

K. Baek, N.P. Balsara; "Controlled Radical Polymerization of Sulfonated Block Copolymers With and Without Protecting Groups," draft to be submitted to Macromolecules.

R. Kostecki, M. Marcinek; "Microwave-Assisted Plasma Deposition of Pt-C Catalyst Films," draft to be submitted to Electrochemical and Solid State Letters.

EETD-Mao LB04021 Nanostructured Cathodes for Efficient Organic LEDs

G. Liu, S. Johnson, J. Kerr, A. Minor, S.S. Mao; "Carbon nanotube as charge injection/collection cathode in organic LED and photovoltaic applications," patent application (2005), a paper will follow after the filing.

S.S. Mao, G. Liu, S. Johnson; "Organic LED devices with structured electrodes," patent application (2005), a paper will follow after the filing. 
EETD-Menon LB04023 Evaluating Aerosol Effects on Regional and Global Energy and Water Budgets

Menon, S. and A.D. Del Genio; "Evaluating the impacts of carbonaceous aerosols on clouds and climate," in "Human-induced climate change: An interdisciplinary Assessment", edited by Schlesinger et al., in press.

Menon, S., and L. Rotstayn; "The radiative influence of aerosol effects on warm cumulus clouds based on sensitivity studies with two climate models," submitted to Climate Change.

Nazarenko, L., and S. Menon; "Varying trends in surface energy fluxes and associated climate between 1960-2002 based on transient climate simulations," Geophys. Res. Lettr., 32, doi:10.1029/2005GL024089 (2005).

Hansen, J., L. Nazarenko, R. Ruedy, et al; “Earth's energy imbalance: Confirmation and implications," Science 308, 1431-1435 (2005).

Hansen, J., Mki. Sato, R. Ruedy, et al; "Efficacy of climate forcings," J. Geophys. Res., 110, doi:101029/2005JD005776 (2005).

Novakov, T., S. Menon, T. Kirchstetter, D. Koch and J. Hansen; "Aerosol organic carbon to black carbon ratios: Analysis of published data and implications for climate forcing," $J$. Geophys. Res., 110, doi:101029/2005JD005977 (2005).

Niyogi, D., H-I. Chang, L. Gu, S. Menon, R.A. Pielke Sr.; "Potential impacts of aerosol-landatmosphere interactions on the Indian monsoonal rainfall characteristics," Natural hazards - Special Issue on Monsoons, in press.

Qian, Y., Q. Song, S. Menon, S. Yu, S. Liu, G. Shi, R. Leung, and Y. Luo; "Impacts of atmospheric sulfate and black carbon aerosols on the regional climate of East Asia," in Global Environmental Change Research in East Asia, in press.

Menon, S.; "Aerosols and Climate change: An Overview," invited talk at Purdue University Fall Seminar Series, Sep 22, 2005, West Lafayette, IN.

Menon, S.; "Regional impacts of aerosols," invited talk at Air Pollution as a Climate Forcing Workshop, April 4-6, 2005, Honolulu, HI.

Menon, S.; "Regional hydrological cycle effects," invited talk at Workshop on Aerosols and the hydrological cycle, July 11-17 2004, Aspen Global Change Institute, Aspen, CO.

Menon, S.; "Aerosol-Cloud Interactions detected by MODIS," invited talk at AEROCOM Workshop, December 1-3, 2004, New York, NY.

Menon, S.; "Detecting black carbon effects," invited talk at Energy Modeling Forum Workshop, July-August 2004, Snowmass, CO.

Menon, S.; "Modeling the indirect aerosol effect," invited talk at AEROCOM Workshop, March 10-12, 2004, Ispra, Italy.

Menon, S.; "What are useful results for climate models," invited talk at Energy Modeling Forum-22, November 10-12, 2004, Brussels, Belgium.

Menon, S., and D. Koch; "Regional and global climate change associated with carbonaceous aerosols," at IAMAS 2005 Scientific Assembly, Aug 2-11 2005, Beijing, China.

Menon, S., and L. Rotstayn; "Effects of aerosols on precipitation in convectively active regions," at $3^{\text {rd }}$ Pan-GCSS meeting on clouds, climate and models, May 16-21 2005, Athens, Greece.

Menon, S.; "Regional impacts of carbonaceous aerosols, " at $8^{\text {th }}$ IGAC Conference, September 4-9 2004, Christchurch, New Zealand. 
EETD-Newman LB05028 Analysis of High-temperature Polymer-electrolyte Fuel Cell Phenomena

A. Weber and J. Newman; "Simulation of high-temperature polymer-electrolyte fuel cells," draft for submission to Journal of the Electrochemical Society.

EETD-Sathaye LB04043 Long-term Global Energy Demand and Carbon Emissions Scenarios

Levine, M.D., Sathaye, J., Price, L., Sinton, J., de la Rue du Can, S., Zhou, N., Worrell, E.; "Disaggregation of Greenhouse Gas Emissions Scenarios: Matching End-Use Technological Information to Projected Trends," draft in preparation for submission to International Journal of Energy Technology and Policy.

Price, L., de la Rue du Can, S., Sinton, J., and Worrell, E.; draft report Sectoral Trends in Global Energy Use and Greenhouse Gas Emissions. Berkeley, CA: Lawrence Berkeley National Laboratory (LBNL-56144).

Sathaye J.; "Bottom-up Modeling of Energy and Greenhouse Gas Emissions: Approaches, Results, and Challenges to Inclusion of End-use Technologies," book chapter in M. Schlesinger (Chief Ed.) Human-Induced Climate Change: An Interdisciplinary Assessment, Cambridge University Press; in press.

GN-Myers LB04026 Making the Most of Sequencing: Improved Assembly and Comparative Annotation

Rasmussen, Kim, Stoye, Jens, Myers, Gene; "Efficient q-gram filters for finding all $\varepsilon$ matches over a given length," Proc. Symp. On Computational Molecular Biology (Boston, MA 2005), 615-629.

Edgar, Robert, Smith, Christopher, Celniker, Susan, Myers, Gene; "PILER: computational identification of novel interspersed repeats," Conf. On Intelligent Systems for Molecular Biology (Detroit, MI 2005), 52-58.

Myers , Gene; “The Fragment Assembly String Graph,” European Conf. on Computational Biology (Madrid, Spain 2005), 79-85.

M. Margulies, M. Egholm, E.W.Myers, R.F. Begley, J.M. Rothberg; "Genome Sequencing in Microfabricated Reactdors," Nature 437, 376-380 (2005).

Brudno, Michael, Davidson, Stuart, and Myers, Gene; "Building String Graphs for Real Data," draft to be presented at "The Intelligent Systems for Molecular Biology Conference" in Brazil, August 2006 (ISMB '06).

Schwartz, Ariel, Myers, Gene, and Pachter, Lior; “Alignment Metric Accuracy,” draft to be presented at "The Intelligent Systems for Molecular Biology Conference" in Brazil, August 2006 (ISMB ’06).

GN-Rubin LB05034 Transgenic Mouse Production and Identification of New Genes in Transgenic Mice

The results of this study are available in a public database (url: http://enhancer.lbl.gov/).

LSD-Auer LB04010 Molecular Electron Microscope Tomograph

M. Auer, A.J. Koster, U. Ziese, N. Volkmann, C. Bajaj, DN Wang, A.J. Hudspeth; "3D visualization of frog hair cell extracellular links by electron microscope tomography," draft in preparation to be submitted to $P N A S$. 
H. Palsdottir, D.N. Wang, A.J. Hudspeth, M. Auer; "Visualizing frog inner ear cell lipid raft by EM tomography," draft in preparation to be submitted to $J C B$.

H. Palsdottir, J.P. Remis, C. Schaudinn, R. Lux, W. Shi, W.B. Costerton M. Auer; "Interbacterial vesicle trafficking in Myxococcus xanthus biofilms accounts for the cell-to-cell transfer of outer membrane proteins," draft in preparation to be submitted to Science.

LSD-Conboy LB05009 Unmasking the Human Genome Alternative Splicing Program

Minovitsky S, Gee SL, Schokrpur S, Dubchak I and Conboy JG; "The splicing regulatory element, UGCAUG, is phylogenetically and spatially conserved in introns that flank tissuespecific alternative exons," Nucl Acids Res.33: 714-724 (2005).

LSD-Glaeser LB05042 Improved Phase Contrast for Cryo-EM of Biological Machines and Subcelluar Structure

R. Cambie, K.H. Downing, D. Typke, R.M. Glaeser, J. Jin; "Design of a Microfabricated, Two-electrode Phase-contrast Element Suitable for Electron Microscopy," draft in preparation.

LSD-Hang LB05040 Computational Analysis of Structural Impacts of Exocyclic DNA Adducts on Specificity and Efficiency of Human Repair Enzymes

P. Wang, A. Guliaev, R. Elder, and B. Hang; "Alkylpurine-DNA-N-glycosylase excision of 7-(hydroxymethyl)-1, $\mathrm{N}^{6}$-ethenoadenine, a glycidaldehyde-derived DNA adduct," DNA Repair, Nov. 10, 2005.

\section{LSD-Jagust LB04014 Neuroimaging with Advanced Molecular Probes}

S. Landau, J. Kramer, S. Baker, J. O’Neil, F. Sun, J. Eberling, M. Oliver, W. Jagust; "Individual variability in dopamine synthesis and working memory function in healthy older adults," poster presentation in Society for Neuroscience Abstracts 537.18 (2005).

F. Sun, S. Baker, J. O’Neil, B. Miller, H. Rosen, W. Jagust; "Distinguishing Alzheimer's disease and frontotemporal dementia using PET imaging with Pittsburgh compound-B," poster presentation in Society for Neuroscience Abstracts 91.3, 2005.

LSD-Martin LB04022 Imaging Signaling Networks in Normal and Malignant Tissue

D. Shvartsman, J. Donaldson, O. Gutman, B. Díaz, G. S. Martin, and Y. Henis; "Association of Src with membrane proteins is dependent on its SH2 and kinase domains," draft to be submitted to J. Cell Biology.

MSD-Dubon LB04008 Ordering and Shape Control of Epitaxial Germanium Nanostructures on Metal-patterened Silicon

J. T. Robinson, J.A. Liddle, A. Minor, V. Radmilovic, and O.D. Dubon; "Morphological evolution of Ge islands on Au-patterned Si," Journal of Crystal Growth, in-press.

J.T. Robinson, J.A. Liddle, A. Minor, V. Radmilovic, D. O. Yi, P. A. Greaney, K. N. Long, D. C. Chrzan, and O.D. Dubon; "Metal-induced assembly of a semiconductor-island 
lattice: Ge truncated pyramids on Au-patterned Si," Nano Letters 5 (10), 2070-2073 (2005).

MSD-Isacoff LB05016 Light-activated and Ion Channels and Transporters

Banghart, M., Borges, K., Isacoff, E.Y., Trauner, D. and Kramer, R.H. (2004). Lightactivated ion channels for remote control of neuronal firing. Nature Neurosci. 7: 1381-6.

MSD-Liddle LB04018 Nanoscale Lighography to Guide Self-Assembly for the Creation of Functional, Hierarchical Nanostructures

Gregg M. Wilmes, David A. Durkee, Nitash P. Balsara, and J. Alexander Liddle; "Investigating the Limits of Surface-Directed Self-Assembly of Pure Block Copolymers," presented at Gordon Conference on Organic Thin Films, August, 2005.

Gregg M. Wilmes, David A. Durkee, Nitash P. Balsara, and J. Alexander Liddle; "Investigating the Limits of Surface-Directed Self-Assembly of Pure Block Copolymers," presented at FENA/IPAM Meeting on Self-Assembly, UCLA, November, 2005.

Gregg M. Wilmes, David A. Durkee, Nitash P. Balsara, and J. Alexander Liddle; "Investigating the Limits of Surface-Directed Self-Assembly of Pure Block Copolymers," presented at MRS Fall Meeting, November, 2005.

Gregg M. Wilmes, David A. Durkee, Nitash P. Balsara, and J. Alexander Liddle; "Bending Soft Block Copolymer Nanostructures by Lithographically Directed Assembly," submitted to Macromolecules.

MSD-Orenstein LB05045 Science and Technology of Quantum Materials

Y. Takamura, R. V. Chopdekar, J. K. Grepstad, Y. Suzuki, A. F. Marshall, A. Vailionis, H. Zheng, J. F. Mitchell; "Thickness Dependent Properties of (110)-Oriented Epitaxial Thin Films of $\mathrm{La}_{1.2} \mathrm{Sr}_{1.8} \mathrm{Mn}_{2} \mathrm{O}_{7}$ Films," to be published in Journal of Applied Physics.

D. Podolsky, A. Vishwanath, J. E. Moore, and S. Sachdev; "Thermoelectric transport near pair breaking quantum phase transition out of d-wave superconductivity," condmat/0510597, submitted to Phys. Rev. Lett.

H. Fu, C. Honerkamp and D.-H. Lee; "A Renormalization Group Study of the Electronphonon Interaction in High-Tc Cuprates,", submitted to Phys. Rev. Lett.

MSD-Suzuki LB04035 Complex Oxide Nanostructures: Understanding Surface, Interface, and Finite Size Effects Through Nanofabrication

Yayoi Takamura, Jostein K. Grepstad, Rajesh V. Chopdekar, Ann F. Marshall, Hong Zheng, John F. Mitchell, and Yuri Suzuki; "Structural, Magnetic and Electronic Properties of (110)-Oriented Epitaxial Thin Films of the Bilayer Magnanite $\mathrm{La}_{1.2} \mathrm{Sr}_{1.8} \mathrm{Mn}_{2} \mathrm{O}_{7}$," Applied Physics Letters 87: 142508 (2005).

Jostein Grepstad, Yayoi Takamura, Andreas Scholl, Ingebrigt Hole, Yuri Suzuki, Thomas Tybell; "Effects of Thermal Annealing in Oxygen on the Antiferromagnetic Order and Domain Structure of Epitaxial $\mathrm{LaFeO}_{3}$ Thin Films," Thin Solid Films 486: 108-112 (2005).

Yayoi Takamura, Rajesh V. Chopdekar, Jostein K. Grepstad, Y. Suzuki, Ann F. Marshall, Arturas Vailionis, Hong Zheng, John F. Mitchell; “Thickness Dependent Properties of 
(110)-Oriented Epitaxial Thin Films of $\mathrm{La}_{1.2} \mathrm{Sr}_{1.8} \mathrm{Mn}_{2} \mathrm{O}_{7}$ Films," to be published in Journal of Applied Physics.

MSD-Vishwanath LB05038 Experimental Signatures of Fractionalized Phases and Phase Transitions in Strongly Correlated Electronic Systems

R. G. Melko, A. Paramekanti, A. A. Burkov, A. Vishwanath, D. N. Sheng, and L. Balents; "Supersolid Order from Disorder: Hard-Core Bosons on the Triangular Lattice," Phys. Rev. Lett. 95: 127207 (2005).

L. Mathey, E. Altman, A. Vishwanath; "Noise Correlations in one-dimensional systems of ultra-cold fermions," Cond-mat/0507108 (preprint), submitted to Physical Review; url: http://arxiv.org/abs/cond-mat/0507108.

L. Capriotti, A. Laeuchli, A. Paramekanti; "Effect of local charge fluctuations on spin physics in the Neel state of $\mathrm{La} \$ 2 \$ \mathrm{CuO} \$ 4 \$$," Cond-mat $/ 0506188$ (preprint), submitted to Physical Review; url: http://arxiv.org/abs/cond-mat/0506188.

NSD-Lesko LB04017 Research and Development for Double Beta Decay Experiments

R. Henning; "The Majorana Neutrinoless Double-Beta Decay Experiment," presented at the American Physical Society Meeting Tampa FL, BAPS H12.00001, 17 April 2005.

R. Henning; "Background Mitigation in a Highly Segmented HPGe Detector," presented at the American Physical Society Meeting, Maui HI, FR.00046, JG.00005, 18-22 September 2005.

R. Henning; "Background Reduction in the Majorana Neutrinoless Double-Beta Decay Experiment," presented at the American Physical Society Meeting, Maui HI, JG.00005, 18-22 September 2005.

K. Hudek, R. Henning, and A. Poon; "Simulation of Cosmogenic Backgrounds at Underground Facilities," presented at the American Physical Society Meeting, Maui, HI, FR.00052, 18-22 September 2005.

K. Lesko; "The Majorana Double-Beta Decay Project," invited talk at the workshop on Double Beta Decay and Neutrino Mass, 2nd Joint Meeting of the Nuclear Physics Divisions of the APS and The Physical Society of Japan; Maui HI, 18-22 September 2005.

T. Gutierrez; "Cuoricino to CUORE: Neutrinoless Double-Beta Decay Measurements with $\mathrm{TeO} 2$ Bolometers," invited talk at the workshop on Double Beta Decay and Neutrino Mass, 2nd Joint Meeting of the Nuclear Physics Divisions of the APS and The Physical Society of Japan; Maui HI, 1WF.00002, 18-22 September 2005.

T. Gutierrez; "Probing the Quantum Nature of the Neutrino Using Two-Particle Interferometry," nucl-th/0510069, submitted to Phys. Rev. Lett.

T. Gutierrez; "Probing the Quantum Nature of the Neutrino with Two-particle Interferometry," presented to the American Physical Society Meeting, Maui HI, F.00006, 18-22 September 2005.

M. Dolinski; "Radioactive Background Studies for the CUORE Neutrinoless Double-Beta Decay Experiment," contributed talk at the 2nd Joint Meeting of the Nuclear Physics Divisions of the APS and The Physical Society of Japan; Maui HI, 22 September 2005.

M. Dolinski; "CUORE Neutrinoless Double-Beta Decay Experiment," presented at meeting of the California Section of the APS, California State University, Sacramento, 21-22 October 2005. 
C. Brofferio, et al; "Cuoricino Status and CUORE Prospects," Nucl. Phys. Proc. Suppl.145: 268-271 (2005).

C. Arnaboldi, et al; "A New Limit on the Neutrinoless Beta Decay of Te-130," Phys. Rev. Lett. 95: 142501(2005).

S. Capelli, et al; "Cuoricino Last Results and CUORE R\&D," presented at the 40th Rencontres de Moriond on Electroweak Interactions and Unified Theories, La Thuile, Aosta Valley,Italy, 5-12 Mar 2005.

R. Ardito, T. Gutierrez, et al; "CUORE: A Cryogenic Underground Observatory for Rare Events," hep-ex/0501010, January 2005.

PBD-Chakraborty LB05006 Organization of Cellular Components on Signaling and Function: Theory and Computation as Essential Complements to Genetic and Biochemical Experiments

P. Schwartz, D. Adalsteinsson, P. Colella, A. P. Arkin, M. Onsum; "Numerical computation of diffusion on a surface," Proc Natl Acad Sci USA 102(32): 11151-6 (2005).

C. V. Rao, J. R. Kirby, \& A. P. Arkin; "Phosphatase localization in bacterial chemotaxis: divergent mechanisms, convergent principles," Phys Biol, 2: 148-158 (2005).

M. D. Onsum, K. Wong, P. Herzmark, H. R. Bourne, A.P. Arkin; "Morphology matters in immune cell chemotaxis: membrane asymmetry affects amplification," submitted Biophysical Journal.

I. Lotan \& T. Head-Gordon; "An analytical electrostatic model for salt screened interactions between multiple proteins," submitted for publication to J. Comp. Theo. Chem.

PBD-Crooks LB05044 Statistical Dynamics of Protein Evolution

G. E. Crooks; "Punctured equilibrium in a model of protein evolution," draft to be submitted to Phys. Rev. E.

G. E. Crooks; "A unification of score functions for the alignment of protein sequence profiles," draft to be submitted to Bioinformatics.

PBD-Fletcher LB05013 Synthetic Cytoskeleton: Protrusive Structures for Reconstituted Cell Motility

A. Liu and D. Fletcher; "Photopatterning of Actin Filament Structures," Nano Letters 5(4): 625-628 (2005).

S. Parekh, O. Chaudhuri, J. Theriot, and D. Fletcher; "Loading History Determines the Velocity of Actin Network Growth," Nature Cell Biology 7(12): pp. 1119-23 (2005).

PD-Battaglia LB05002 Advanced Monolithic Silicon Pixel Sensors

M. Battaglia et al; "Monolithic CMOS Pixel R\&D for the ILC at LBNL," in press for the Proceedings of the 2005 International Linear Collider Workshop (LCWS 2005), Sanford CA, 18-22 March 2005; at url: ArXiv: physics/0508138. 
PD-Heeger LB04012 Designing a Novel Reactor Neutrino Oscillation Experiment for Measuring the Unknown Mixing Angle Theta13

\section{Publications}

Y.X. Sun, J. Cao, K.B. Luk, Y.Q. Ma, Y.F. Wang, C.G. Yang; "Baseline Optimization of Reactor Neutrino Experiments," High Energy Phys. Nucl. Phys. 29: 543 (2005).

B.E. Berger et al; "A Precision Measurement of $\sin ^{2} 2 q_{13}$ with Reactor Antineutrinos at Daya Bay," report submitted by the US Collaborators of the Daya Bay Experiment to the Neutrino Science Assessment Group (NuSAG), May 27 2005; url: http://theta13.lbl.gov/nusag/.

P. Dobson and S. Nakagawa; "Summary of Rock-Property Measurements for Hong Kong Tuff Samples,” LBNL Technical Report, September 2005, LBNL-58878.

\section{Talks and Presentations}

S.J. Freedman; "Measuring $\sin ^{2} 2 \theta_{13}$ at Daya-Bay-Ling Ao," $4^{\text {th }}$ Workshop on Future LowEnergy Neutrino Experiments, Angra dos Reis, RJ, Brazil, 23-25 February 2005; url: http://www.ifi.unicamp.br/ lenews05/programws05.html.

K.M Heeger; "Measuring $\sin ^{2} 2 \theta_{13}$ with Reactor Antineutrinos at Daya Bay," presented at the joint APS/JPS Meeting of the Division of Nuclear Physics, Maui HI, September 17-22 2005.

K.M. Heeger; "Measuring $\sin ^{2} 2 \theta_{13}$ with Reactor Antineutrinos at Daya Bay," presented at the Experimental Physics Seminar at SLAC, Menlo Park CA, July 72005.

K.-B. Luk; "Neutrino Oscillation Experiments with the Daya Bay Nuclear Power Facility," presented at the Neutrino Satellite Meeting at PANIC05, Santa Fe NM, October 30 2005; url: http://www.panic05.lanl.gov/neutrino/neutrino.html.

K.-B. Luk; "Determining the Neutrino Mixing Angle Theta 13 with the Daya Bay Nuclear Power Plants," BNL Particle Physics Seminar, November 4 2005; url: http://www.phy.bnl.gov/ partsem/05-06.html.

PD-Murayama LB05027 New Directions for Theoretical Physics at the TeV-Scale

Dah-Wei Chiou, Ori J. Ganor, Yoon Pyo Hong, Bom Soo Kim, Indrajit Mitra; "Massless And Massive Three Dimensional Super Yang-Mills Theory And Mini-Twistor String Theory," UCB-PTH-04-37, LBNL-56763, Feb 2005.

Indrajit Mitra, Raghunath Ratabole, H.S. Sharatchandra; "Infrared Behaviour Of Massless QED In Space-Time Dimensions $2<$ D < 4," IMSC-2004-10-35, Oct 2004; published in Phys.Lett.B 611: 289-296 (2005).

Veronika E. Hubeny, Mukund Rangamani, Simon F. Ross; "Causal Structures And Holography,” DCPT-05-15, UCB-PTH-05-07, LBNL-57390, Apr 2005.

Veronika E. Hubeny, Mukund Rangamani, Simon F. Ross; "Causally Pathological Spacetimes Are Physically Relevant,” DCPT-05-16, UCB-PTH-05-08, LBNL-57391, Apr 2005.

Veronika Hubeny, Alexander Maloney, Mukund Rangamani; "String-Corrected Black Holes," SLAC-PUB-10879, UCB-PTH-04-34, LBNL-56671, NSF-KITP-04-129, Nov 2004. 
Cross-Div.-Alivisatos LB05001 Studies of the Health Effects of Nanostructured Materials

R.L. Maddalena, M.J. MacLeod, T.E. McKone and M.D. Sohn; "Prospective Hazard Assessment and Sensitivity Analysis for Buckminsterfullerene in the Environment," to be submitted to Integrated Environmental Assessment and Management; LBNL-57769.

L.S. Ruzer and M.G. Apte; "Radon Progeny as a Potential Experimental Tool in Dosimetry of Nanoaerosols," to be submitted to Inhalation Toxicology; LBNL-59256.

L.S. Ruzer and M.G. Apte; "Nanoparticles: A Road Map for Developing a Dosimetry Protocol," to be submitted to Environmental Science and Technology.

L. Ding, J. Stilwell, T. Zhang, O. Elboudwarej, H. Jiang, J.P. Selegue, P.A. Cooke, J.W. Gray, \& F.F. Chen; "Molecular Characterization of the Cytotoxic Mechanism of Multiwall Carbon Nanotubes and Nano-Onions on Human Skin Fibroblast , "Nano Letters 5: 2448-2464 (2005).

T. Zhang, D. Gerion, J. Stilwell, L. Ding, O. Elboudwarej, P.A. Cook, J.W. Gray, A.P. Alivisatos, and F.F. Chen; draft to be submitted to Nano Letters.

F.F. Chen, in USPTO vol. provisional, LBNL Docket ID: IB-2218P, in press.

X. Chen, U.C. Tam, J. L. Czlapinski, G.S Lee, D. Rabuka, A. Zettl, \& C.R. Bertozzi; "Interfacing Carbon Nanotubes with Living Cells," to be submitted to J. Am. Chem. Soc.

Cross-Div.-Geissler LB03035 Microscopic Theory of Protein Surface: Structure, Response, and Design

K. DuBay and P. Geissler; "Spatial correlations of side chain rotations in photoactive yellow protein due to steric constraints," draft to be submitted to Journal of Molecular Biology.

Cross-Div.-Lester LB05020 Development and Application of Quantum Monte Carlo (QMC) Methods to Biological Systems

R. Salomon-Ferrer, B. Austin, A. Aspuru-Guzik, G. Fleming, M. Frenklach, and W. A. Lester, Jr.; "Electron Distributions and Bonding in Ground Singlet and Lowest Triplet States of Bacteriochlorophl and Speroidene," to be prepared.

B. Austin, R. Salomon-Ferrer,, A. Aspuru-Guzik, G. Fleming, M. Frenklach, and W. A. Lester, Jr.; "Low-lying Energy Levels of Bacteriochlorophl and Speroidene," to be prepared.

Cross-Div.-Marx LB05025 Development of Key Physics and Technical Approaches for the Production of Intense Rare Isotope Beams

D. Todd, D. Leitner, D. Grote, M. Leitner, C. Lyneis and J. Qiang; "Comparison of Beam Transport Simulations with Experiments for the SC ECR Ion Source VENUS," contributed Paper, ICIS'05, RSI, submitted 2005.

D. Todd, D. Leitner, J. Qiang, C. Lyneis, D. Grote; "Particle-in-Cell Simulations of the VENUS Ion Beam Transport System, Contributed Paper," published in the Proceedings of the 2005 Particle Accelerator Conference, Knoxville, Tennessee, 2005; url http://accelconf.web.cern.ch/accelconf/p05/PAPERS/TPPE032.PDF.

J. Qiang, D. Leitner, D.S. Todd; "A Parallel 3d Model for The Multi-Species Low Energy Beam Transport System of the RIA Prototype ECR Ion Source Venus," contributed paper, Proceedings of the 2005 Particle Accelerator Conference, Knoxville, Tennessee, 2005; url: http://accelconf.web.cern.ch/accelconf/p05/PAPERS/TPPE010.PDF. 
D. Leitner, C. M. Lyneis; "R\&D Efforts at LBNL on the Injector Ion Source and Low Energy Beam Transport for the RIA Driver LINAC," LBNL-RIA Technical Note, LBNL-RIA $T N-D F-001,11$ November 2004.

D. Li, J. Staples, S. Virostek; "Detailed Modeling of the SNS RFQ Structure With CST Microwave Studio," 2006 International Linear Accelerator Conference, Knoxville, TN, 21-25 August 2006. 